\title{
Investigating the Underappreciated Hydrolytic Instability of DBU and Related Unsaturated Nitrogenous Bases
}

Alan M. Hyde, * Ralph Calabria, Rebecca Arvary, Xiao Wang and Artis Klapars

Department of Process Research \& Development, MRL, Merck \& Co., Inc., 126 E. Lincoln Ave. Rahway, New Jersey 07065, United States

*E-mail: alan_hyde@merck.com.

\section{Contents}

Base Screening Experiments for the Conversion of Methyluridine 6 to Anhydrouridine 8a .................... 2

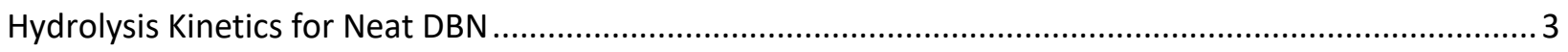

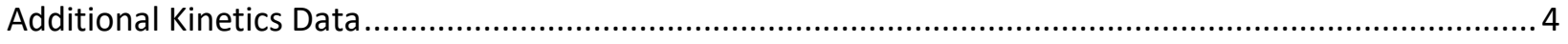

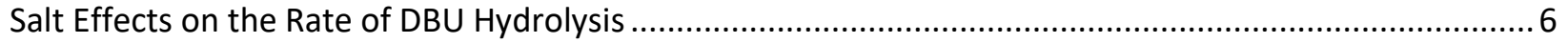

Calculated Structures Related to DBU and DBN Hydrolysis................................................................... 7

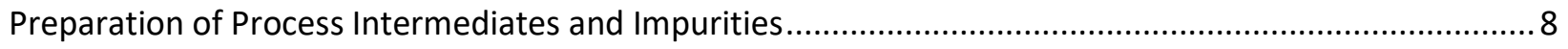

NMR Spectra of Process Intermediates and Impurities ........................................................................ 10 


\section{Base Screening Experiments for the Conversion of Methyluridine 6 to Anhydrouridine 8a}

A stock solution containing methyluridine 6 (800 mg, $3.1 \mathrm{mmol}$ ) and CDI (653 mg, $4.03 \mathrm{mmol}$ ) in MeCN $(5.76 \mathrm{~mL})$ was added to a $40 \mathrm{~mL}$ scintillation vial containing the indicated base under a nitrogen atmosphere. The reactions were heated to $80^{\circ} \mathrm{C}$ for $15 \mathrm{~h}$. The visual appearance was noted at the beginning and at the end. Yields were determined by taking a measured aliquot and diluting to a known volume in a volumetric flask using $0.1 \mathrm{M} \mathrm{H}_{3} \mathrm{PO}_{4}$. If solids were present, DMSO was also added.

Table S1. Screening bases for generation of $\mathbf{8 a}$ and physical properties of reaction/product mixture.<smiles>[Y4][C@]1(O)[C@@H](O)[C@@H](CO)O[C@H]1n1ccc(=O)[nH]c1=O</smiles>

6

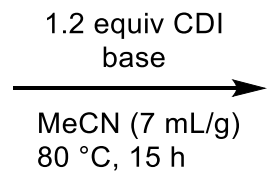

$80^{\circ} \mathrm{C}, 15 \mathrm{~h}$<smiles>N[C@@]12Oc3nc(=O)ccn3[C@@H]1O[C@H](CO)[C@@H]2O</smiles>

$8 \mathbf{a}$

\begin{tabular}{|c|c|c|c|c|}
\hline Entry & Base & Equiv & Visual Observations & \% LC Yield \\
\hline 1 & $\mathrm{KOH}$ & 0.2 & crusting/oiling & 75.2 \\
\hline 2 & $\mathrm{CsOH} \cdot \mathrm{H}_{2} \mathrm{O}$ & 0.2 & crusting/oiling & 88.7 \\
\hline 3 & $\mathrm{Mg}(\mathrm{OH})_{2}$ & 0.2 & crusting/oiling & 4.1 \\
\hline 4 & $\mathrm{CsF}$ & 0.2 & crusting/oiling & 45.4 \\
\hline 5 & $\mathrm{LiO} t-\mathrm{Bu}$ & 0.2 & crusting/oiling & 15.5 \\
\hline 6 & $\mathrm{KO} t-\mathrm{Bu}$ & 0.2 & crusting/oiling & 50.9 \\
\hline 7 & $\mathrm{NaH}$ & 1 & crusting/oiling & 90.4 \\
\hline 8 & KHMDS & 1 & crusting/oiling & 27.9 \\
\hline 9 & $\mathrm{Bu}_{2} \mathrm{Mg}$ & 1 & crusting/oiling & 14.1 \\
\hline 10 & $i-\operatorname{PrMgCl} \cdot \mathrm{LiCl}$ & 1 & crusting/oiling & 1.4 \\
\hline 11 & $\mathrm{Me}_{4} \mathrm{NOH}$ & 0.2 & homogeneous $\rightarrow$ slurry & 79.1 \\
\hline 12 & $\mathrm{Bu}_{4} \mathrm{NOH}$ in $\mathrm{MeOH}$ & 0.2 & homogeneous $\rightarrow$ slurry & 87.2 \\
\hline 13 & $\mathrm{Et}_{3} \mathrm{~N}$ & 1 & slurry $\rightarrow$ homogeneous & 25 \\
\hline 14 & $\mathrm{Oct}_{3} \mathrm{~N}$ & 1 & crusting/oiling & 13.8 \\
\hline 15 & DBU & 0.2 & homogeneous & 90 \\
\hline
\end{tabular}


Following a first-pass screen in $\mathrm{MeCN}$, the best bases we re-examined in four different solvents. Only $\mathrm{Bu}_{4} \mathrm{NOH}$ and DBU gave consistently good physical properties (i.e., a free-flowing slurry or homogeneous). The use of hydroxide bases generated an additional impurity which is the 2'-epimer of methyluridine 6 arising from hydrolysis of anhydrouridine $\mathbf{8 a}$.

Table S2. Testing solvent/base combinations based on the best results from Table S1.

\begin{tabular}{|c|c|c|c|c|}
\hline & DME & MeTHF & MeCN & MeCN/IPA (4:1) \\
\hline $\mathbf{K O H}+\mathrm{Bu}_{4} \mathbf{N B r}$ & 66 & 35 & 75 & \\
\hline $\mathbf{C s O H} \cdot \mathbf{H}_{\mathbf{2}} \mathbf{O}$ & 46 & 6 & 63 & 70 \\
\hline $\mathrm{Me}_{\mathbf{4}} \mathbf{N O H}$ & 79 & 48 & 79 & 80 \\
\hline $\mathrm{Bu}_{4} \mathbf{N O H}$ & 67 & 50 & 76 & 78 \\
\hline $\mathrm{DBU}$ & 56 & 65 & 90 & 88 \\
\hline
\end{tabular}

\section{Hydrolysis Kinetics for Neat DBN}

The degradation of DBN was monitored by GC in the same manner that was done for DBU in the main text. Overall, hydrolysis of DBN over the same time period with similar water contents was slower that for DBU. Although we do not have an understanding for why this is the opposite ordering of what was observed in $\mathrm{D}_{2} \mathrm{O}$ solution, it may be related to the difference in mechanism we found for the two bases.

Figure S1. Hydrolysis of neat DBN with variable $\mathrm{H}_{2} \mathrm{O}$ content over two months as measured by GC.

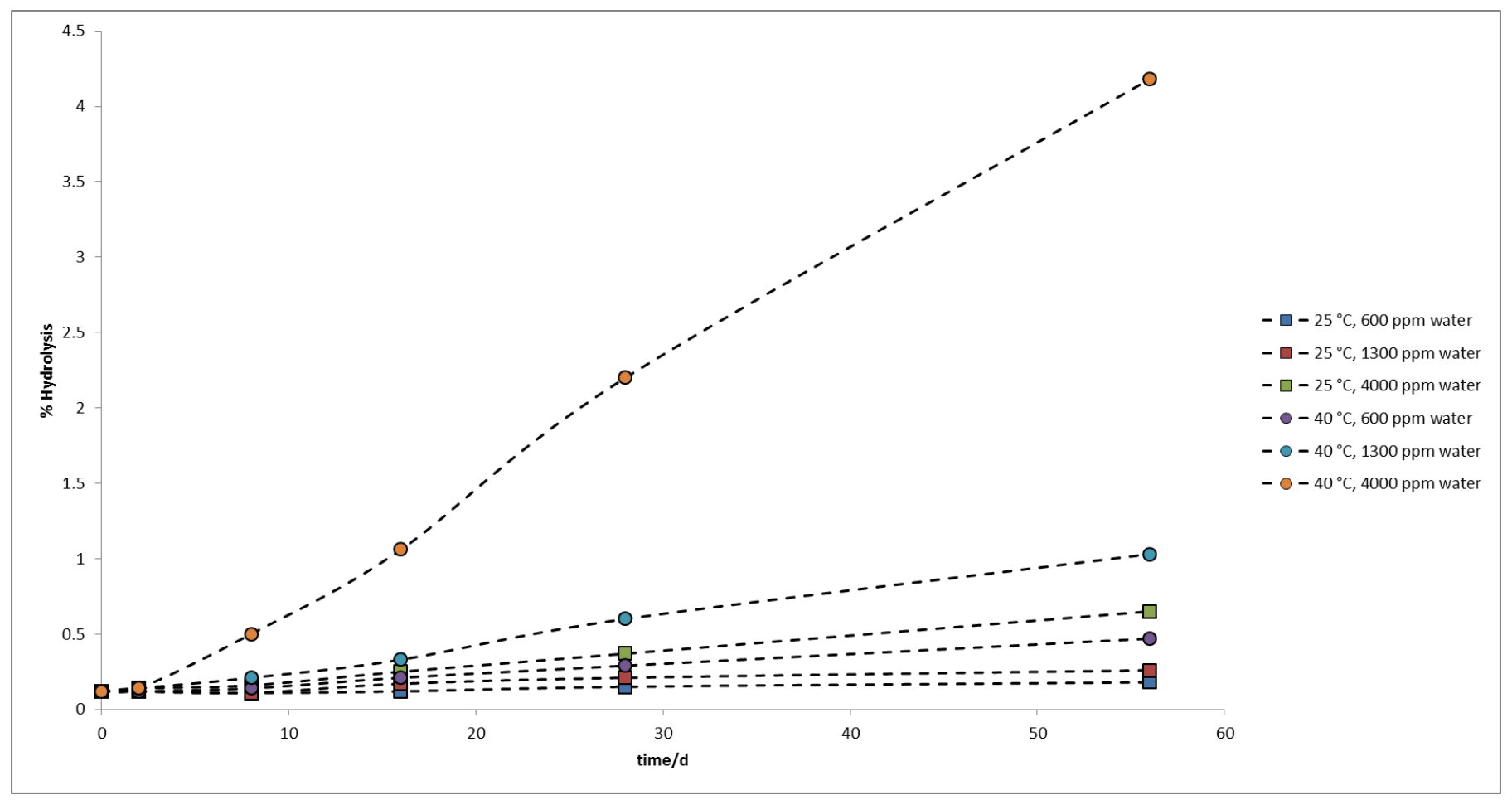




\section{Additional Kinetics Data}

Figure S2. Plot of $\ln ([D B U])$ vs. time in unbuffered $D_{2} \mathrm{O}$ at $27^{\circ} \mathrm{C}$.

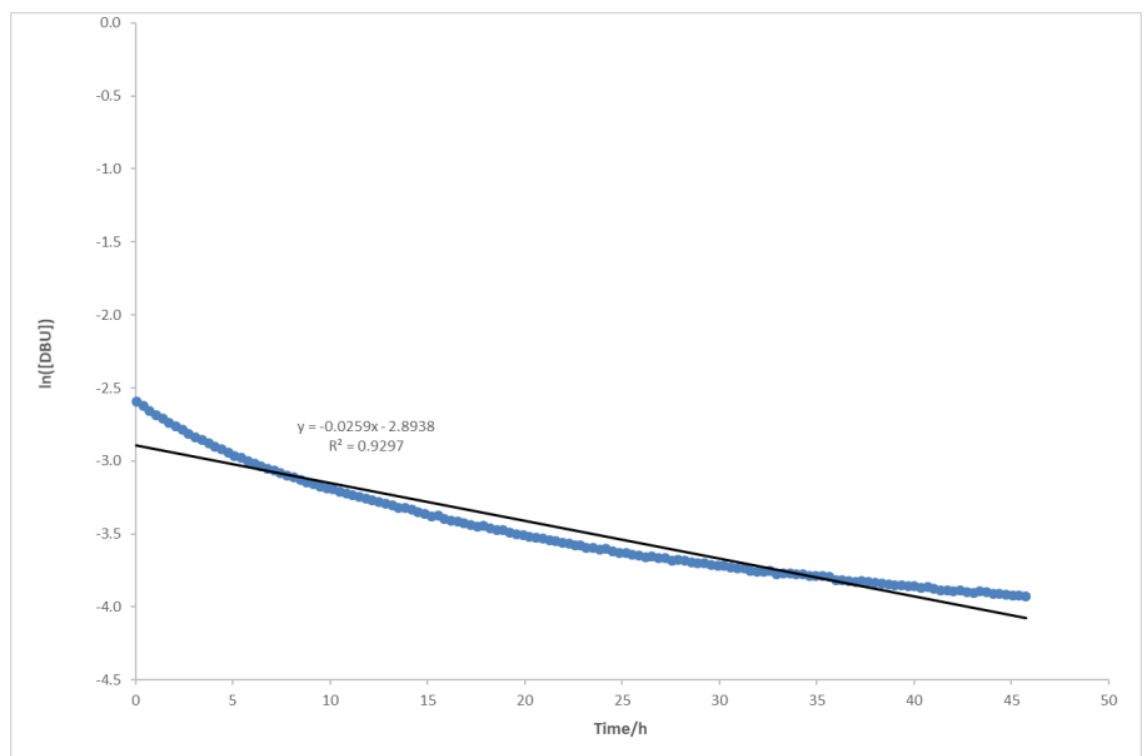

Figure S3. Plot of $\ln ([\mathrm{DBU}])$ vs. time in $\mathrm{pH} 13.5$ buffer at $27^{\circ} \mathrm{C}$.

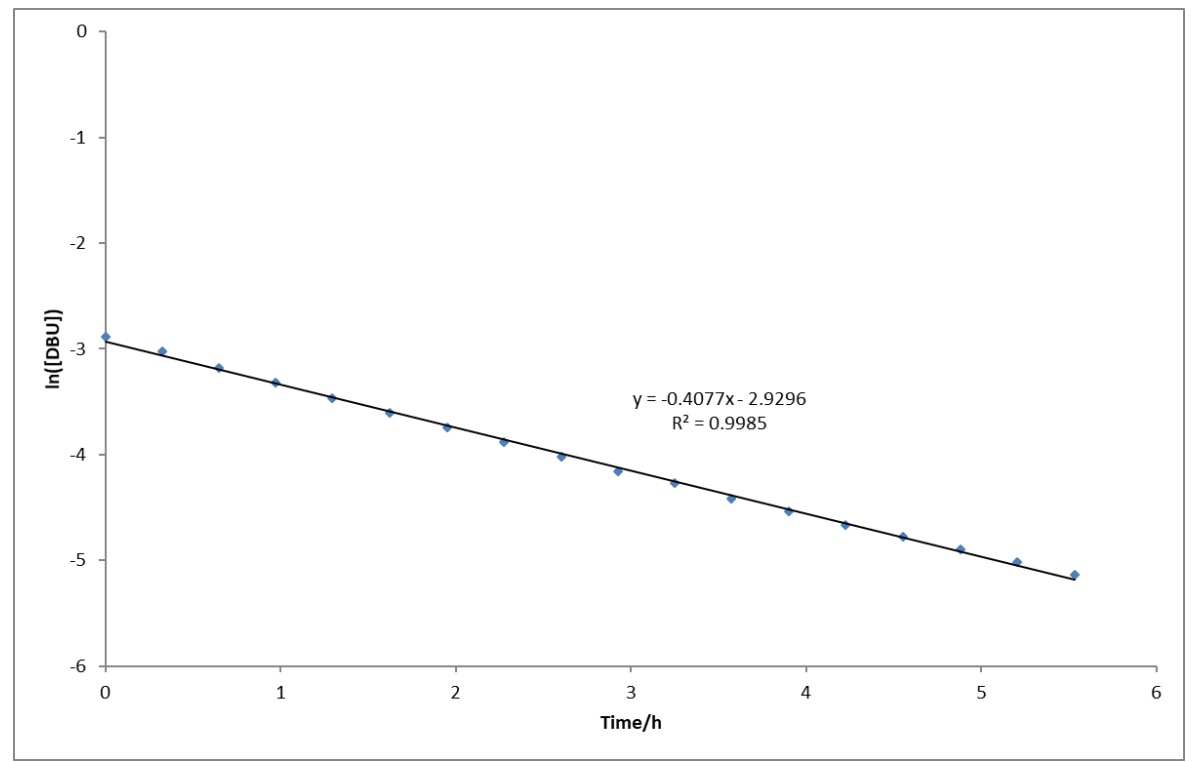


Figure S4. Profile for DBU hydrolysis in 2:1 $d_{3}-\mathrm{MeCN} / \mathrm{D}_{2} \mathrm{O}$

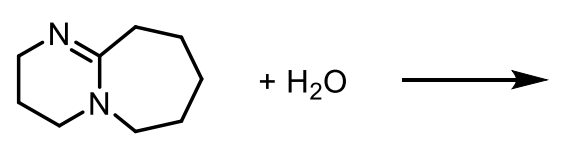

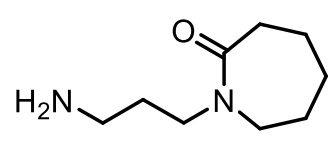

1

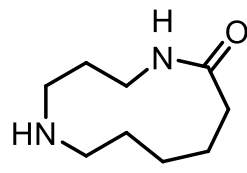

12

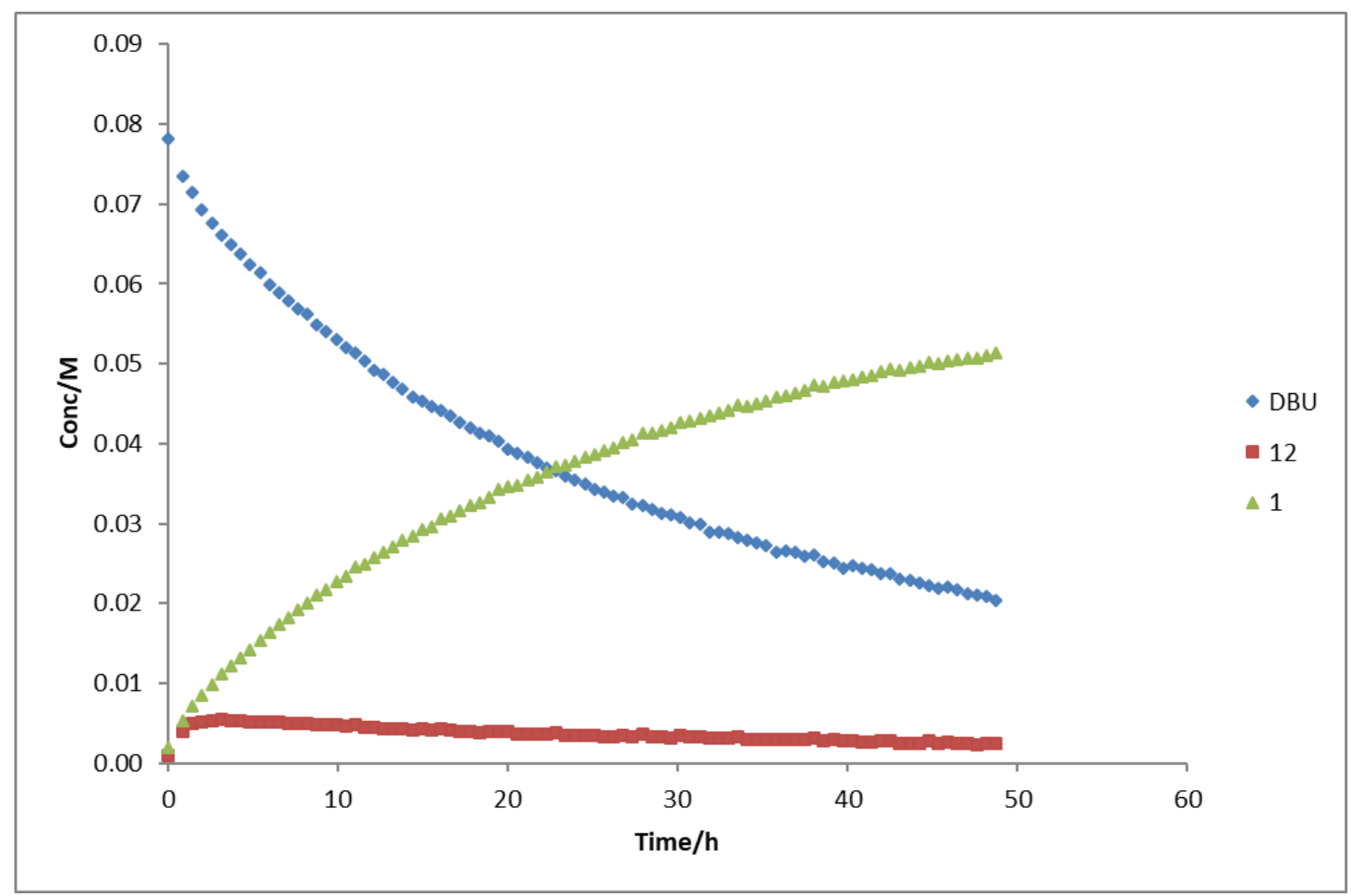




\section{Salt Effects on the Rate of DBU Hydrolysis}

No clear trends were observed in the continuum between kosmotropes $\left(\mathrm{Na}_{2} \mathrm{SO}_{4}\right)$ and chaotropes $\left(\mathrm{NaClO}_{4}\right)$. Overall, we consider this to generally respond to solution non-ideality as predicted by DebyeHuckel theory.

Figure S5. The effect of different salts on the rate of DBU hydrolysis as a test for specific ion effects.

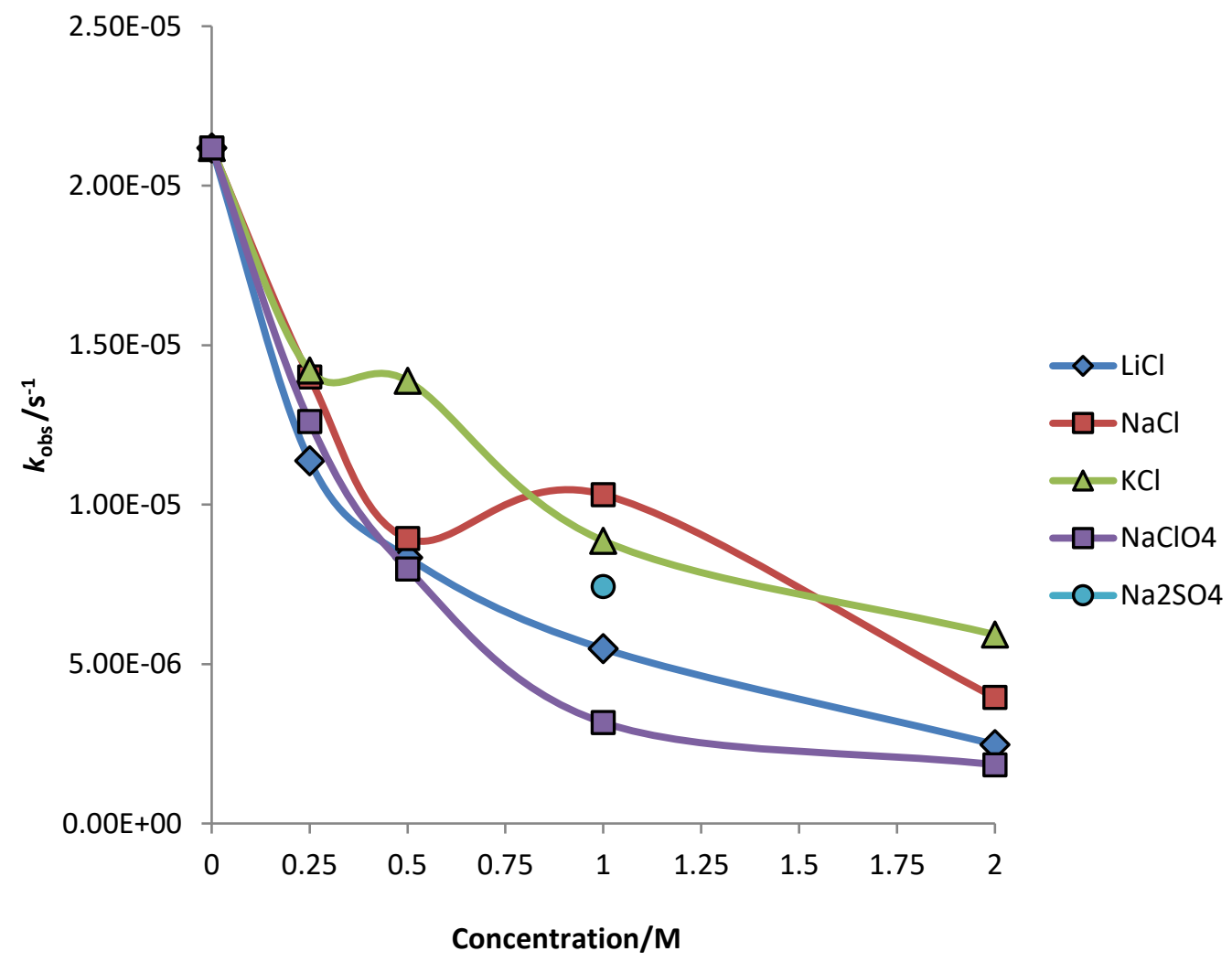




\section{Calculated Structures Related to DBU and DBN Hydrolysis}

All calculations wer performed using Spartan '18, version 1.2.0.

A conformational seach for each structure using the MMFF molecular mechanics model was first performed. Geometry optimization of the lowest energy confomer was performed with M06-2x/6$31 \mathrm{~g}(\mathrm{~d})$ and a water polarization continuum solvent model. Thermochemical data was obtained by calculation of the analytical force constants and with a zero-point energy correction.

Table S3. Calculated energies for the hydrolysis of DBU to $\mathbf{1}$ and $\mathbf{1 2 .}$

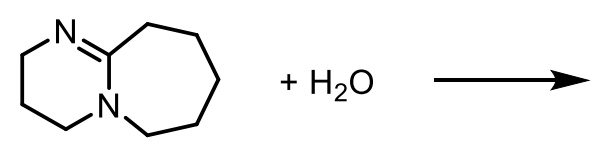

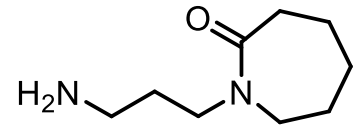

1<smiles>O=C1CCCCCNCCCN1</smiles>

12

\begin{tabular}{|c|c|c|c|}
\hline product & $\Delta \Delta \mathrm{E}(\mathrm{kcal} / \mathrm{mol})$ & $\Delta \Delta \mathrm{H}(\mathrm{kcal} / \mathrm{mol})$ & $\Delta \Delta \mathrm{G}(\mathrm{kcal} / \mathrm{mol})$ \\
\hline $\mathbf{1}$ & -18.5 & -16.0 & -6.2 \\
\hline $\mathbf{1 2}$ & -17.1 & -14.2 & -3.9 \\
\hline
\end{tabular}

Table S4. Calculated energies for the hydrolysis of DBN to $\mathbf{2}$ and $\mathbf{1 3 .}$<smiles>C1CN=C2CCCN2C1</smiles>

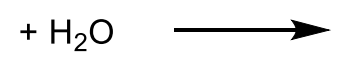<smiles>NCCCN1CCCC1=O</smiles><smiles>O=C1CCCNCCCN1</smiles>

13

\begin{tabular}{|c|c|c|c|}
\hline product & $\Delta \Delta \mathrm{E}(\mathrm{kcal} / \mathrm{mol})$ & $\Delta \Delta \mathrm{H}(\mathrm{kcal} / \mathrm{mol})$ & $\Delta \Delta \mathrm{G}(\mathrm{kcal} / \mathrm{mol})$ \\
\hline $\mathbf{1}$ & -19.2 & -17.0 & -8.3 \\
\hline 13 & -11.9 & -8.4 & +3.1 \\
\hline
\end{tabular}




\section{Preparation of Process Intermediates and Impurities}

Scheme S1. Synthesis and isolation of carbonate intermediates $7 \mathbf{a}$ and $\mathbf{7 b}$

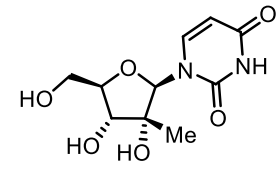

6

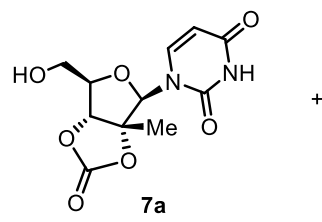

$7 \mathbf{a}$

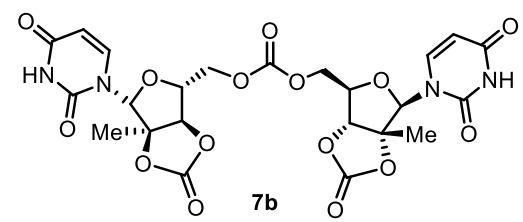

To a mechanically agitated slurry of methyluridine $6(30.0 \mathrm{~g}, 116 \mathrm{mmol})$ in dry acetonitrile $(150 \mathrm{~mL})$ was added solid CDI (18.8 g, $116 \mathrm{mmol}$ ) in one portion resulting in a homogeneous solutuion after about five minutes. The reaction was aged $14 \mathrm{~h}$, providing a white suspension. The solvent was removed by rotary evaporator and the resulting thick slurry was diluted with $1 \mathrm{M}$ aq $\mathrm{H}_{3} \mathrm{PO}_{4}(58 \mathrm{~mL})$ and further acidified with $12 \mathrm{~mL} 85 \% \mathrm{H}_{3} \mathrm{PO}_{4}$. The solids (7b) were filtered and the cake was rinsed with water (2x50 mL).

The filtrate was extracted with 2-MeTHF $(3 \times 100 \mathrm{~mL})$ and the combined organic phase was then washed with $20 \mathrm{wt} \% \mathrm{NaCl}$ (aq, $10 \mathrm{~mL}$ ). The organic phase was concentrated to $100 \mathrm{~mL}$ and seeded with 7a. The slurry was concentrated to $\sim 50 \mathrm{~mL}$. The solids were filtered, washed with 2-MeTHF $(3 \times 15 \mathrm{~mL})$, then dried with nitrogen to give $7 \mathrm{a}(7.4 \mathrm{~g}, 22 \%)$ as a white powder.

Recrystallization of crude $\mathbf{7 b}$ was accomplished by first dissolving in DMF $(30 \mathrm{~mL})$ at $60{ }^{\circ} \mathrm{C}$. Water $(15$ $\mathrm{mL}$ ) was added to first generate a slurry. More water $(15 \mathrm{~mL})$ was added slowly over $1 \mathrm{~h}$ followed by cooling to rt. The solids were filtered, rinsed with water, and dried under nitrogen to provide $\mathbf{7 b}(12.1 \mathrm{~g}$, $35 \%)$ as a white solid.

1-((3aR,4R,6R,6aR)-6-(hydroxymethyl)-3a-methyl-2-oxotetrahydrofuro[3,4-d][1,3]dioxol-4-yl)pyrimidine2,4(1H,3H)-dione (7a): ${ }^{1} \mathrm{H}$ NMR (DMSO- $d_{6}, 600 \mathrm{MHz}, 49^{\circ} \mathrm{C}$ ) $\delta: 11.52(\mathrm{~s}, 1 \mathrm{H}), 7.78(\mathrm{~d}, J=8.2 \mathrm{~Hz}, 1 \mathrm{H}$ ), 6.17 $(\mathrm{s}, 1 \mathrm{H}), 5.69(\mathrm{~d}, J=8.1 \mathrm{~Hz}, 1 \mathrm{H}), 4.88(\mathrm{~d}, J=4.3 \mathrm{~Hz}, 1 \mathrm{H}), 4.30(\mathrm{~d}, J=3.9 \mathrm{~Hz}, 1 \mathrm{H}), 3.78-3.66(\mathrm{~m}, 2 \mathrm{H}), 1.36$ (s, $3 H) .{ }^{13} \mathrm{C}$ NMR $\left(150 \mathrm{MHz}\right.$, DMSO-d $\left.d_{6}\right) \delta: 162.65,152.67,149.96,101.45,83.02,74.01,66.45,59.85,32.41$, 25.11, 20.62, 17.35.

bis(((3aR,4R,6R,6aR)-6-(2,4-dioxo-3,4-dihydropyrimidin-1(2H)-yl)-6a-methyl-2-oxotetrahydrofuro[3,4d][1,3]dioxol-4-yl)methyl) carbonate (7b): ${ }^{1} \mathrm{H}$ NMR (DMSO- $\left.d_{6}, 600 \mathrm{MHz}\right) \delta: 11.47(\mathrm{~s}, 1 \mathrm{H}), 7.60$ (d, $J=8.1$ $\mathrm{Hz}, 1 \mathrm{H}), 6.16(\mathrm{~s}, 1 \mathrm{H}), 5.70(\mathrm{~d}, J=8.1 \mathrm{~Hz}, 1 \mathrm{H}), 4.96(\mathrm{~d}, J=4.6 \mathrm{~Hz}, 1 \mathrm{H}), 4.6-4.38(\mathrm{~m}, 3 \mathrm{H}), 3.22(\mathrm{~s}, 2 \mathrm{H}), 1.40$ $(s, 3 H) .{ }^{13} \mathrm{C}$ NMR (DMSO- $d_{6}, 150 \mathrm{MHz}$ ) $\delta: 162.94,153.79,152.69,150.22,102.05,17.97$. 
Scheme S2. Synthesis and isolation of carbonate intermediate $\mathbf{8 b}$

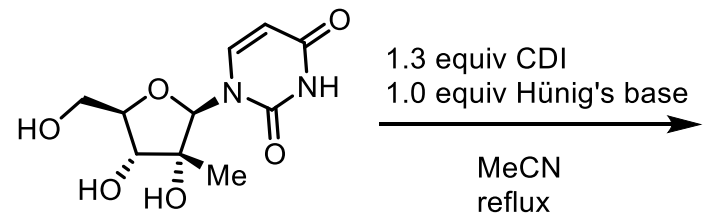

6

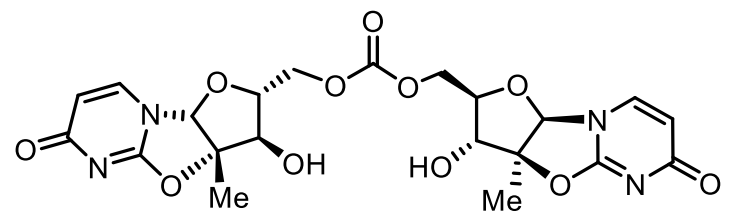

$8 b$

A $40 \mathrm{~mL}$ scintillation vial with magnetic stir bar was charged with methyluridine $6(1.50 \mathrm{~g}, 5.81 \mathrm{mmol})$, acetonitrile $(10.5 \mathrm{~mL})$, and CDI $(1.22 \mathrm{~g}, 7.55 \mathrm{mmol})$. Hunig's base $(0.75 \mathrm{~g}, 5.81 \mathrm{mmol})$ was added and the mixture was heated at $80^{\circ} \mathrm{C}$ for $3 \mathrm{~d}$. MTBE (14 mL) was added to preciptate out anhydrouridine $8 \mathrm{a}$. The filtrate was concentrated to provide $\mathbf{8 b}$ as a colroless solid (yield not determined). bis(( $2 R, 3 R, 3 \mathrm{aS}, 9 \mathrm{a} R)$ 3-hydroxy-3a-methyl-6-oxo-2,3,3a,9a-tetrahydro-6H-furo[2',3':4,5]oxazolo[3,2-a]pyrimidin-2-yl)methyl) carbonate (8b): ${ }^{1} \mathrm{H}$ NMR (Acetonitrile- $d_{3} /$ DMSO- $\left._{6}, 600 \mathrm{MHz}\right) \delta: 7.64-7.56(\mathrm{~m}, 3 \mathrm{H}), 7.03(\mathrm{~d}, \mathrm{~J}=1.1 \mathrm{~Hz}$, $3 \mathrm{H}), 5.94(\mathrm{~d}, J=7.5 \mathrm{~Hz}, 1 \mathrm{H}), 5.83(\mathrm{~s}, 1 \mathrm{H}), 4.41(\mathrm{~d}, J=4.4 \mathrm{~Hz}, 1 \mathrm{H}), 4.22(\mathrm{q}, J=4.5 \mathrm{~Hz}, 1 \mathrm{H}), 4.14$ (dd, J = 11.9, $4.2 \mathrm{~Hz}, 1 \mathrm{H}$ ), $4.08\left(\mathrm{dd}, J=11.9,5.0 \mathrm{~Hz}, 1 \mathrm{H}\right.$ ), $1.62(\mathrm{~s}, 3 \mathrm{H}) .{ }^{13} \mathrm{C}$ NMR (Acetonitrile- $d_{3} / \mathrm{DMSO} d_{6}, 150 \mathrm{MHz}$ ) $\delta$ : 160.42 , 137.46, 136.11, 110.10, 96.39, 94.68, 85.86, 77.14, 67.57, 40.55, 40.41, 40.27, 40.13, 17.45, $1.73,1.60,1.04$.

Scheme S3. Synthesis and isolation of carbamate impurity 9
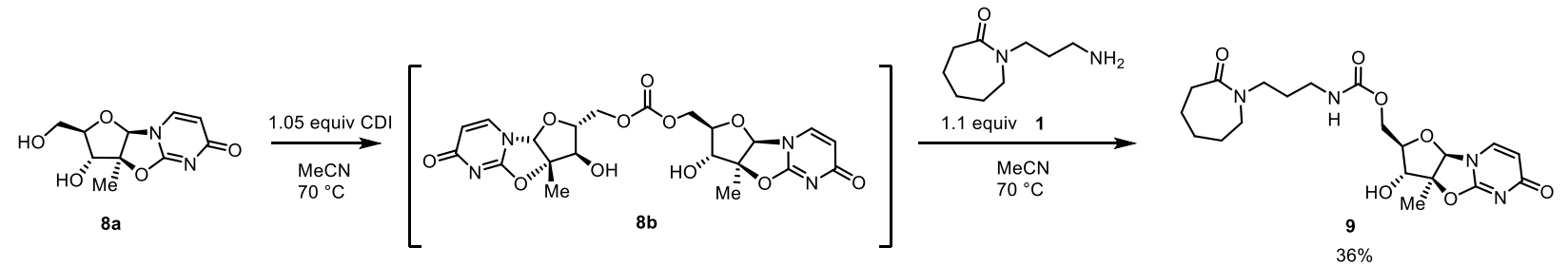

A $40 \mathrm{~mL}$ scintillation vial with magnetic stir bar was charged with anhydrouridine $8 \mathrm{a}(1.0 \mathrm{~g}, 4.16 \mathrm{mmol})$, acetonitrile $(20 \mathrm{~mL})$, and CDI $(0.658 \mathrm{~g}, 4.37 \mathrm{mmol})$. The mixture was heated at $70^{\circ} \mathrm{C}$ for $15 \mathrm{~h}$, and the slurry was cooled to rt. Amine $1(0.78 \mathrm{~g}, 4.57 \mathrm{mmol})$ was charged and the reaction was heated at $70{ }^{\circ} \mathrm{C}$ overnight. The solids (mostly unreacted $\mathbf{8 a}$ ) were filtered and the filtrate was concentrated by rotary evaporator. The residue was purified by column chromatography (normal phase silica, ISCO) with a gradient of $0-20 \% \mathrm{MeOH}$ in DCM. The desired product 9 was isolated as a colorless oil. $650 \mathrm{mg}, 36 \%$ yield. ((2R,3R,3aS,9aR)-3-hydroxy-3a-methyl-6-oxo-2,3,3a,9a-tetrahydro-6H-furo[2',3':4,5]oxazolo[3,2a]pyrimidin-2-yl)methyl (3-(2-oxoazepan-1-yl)propyl)carbamate (9): ${ }^{1} \mathrm{H}$ NMR (DMSO- $\left.d_{6}, 600 \mathrm{MHz}\right) \delta$ : $7.82(\mathrm{~d}, J=7.3 \mathrm{~Hz}, 1 \mathrm{H}), 7.06(\mathrm{t}, J=5.8 \mathrm{~Hz}, 1 \mathrm{H}), 5.86-5.91(\mathrm{~m}, 2 \mathrm{H}), 4.16(\mathrm{~d}, J=5.5 \mathrm{~Hz}, 1 \mathrm{H}), 4.12(\mathrm{dd}, J=$ 12.1, 3.4 Hz, 1H), 4.04-4.09 (m, 2H), 3.88 (dd, J = 12.1, 6.7 Hz, 1H), 3.27-3.33 (m, 2H), 3.23 (t, J = 7.1 Hz, $2 \mathrm{H}), 2.92(\mathrm{q}, J=6.7 \mathrm{~Hz}, 2 \mathrm{H}), 2.35-2.43(\mathrm{~m}, 2 \mathrm{H}), 1.59-1.67(\mathrm{~m}, 2 \mathrm{H}), 1.44-1.57(\mathrm{~m}, 9 \mathrm{H}) .{ }^{13} \mathrm{C}$ NMR (DMSO$d_{6}, 150 \mathrm{MHz}$ ): 国: 174.9, 171.4, 159.3, 155.7, 137.1, 109.0, 95.1, 92.7, 83.8, 76.0, 63.1, 48.7, 45.1, 38.2, $36.6,29.3,28.4,28.1,23.1,16.8$. 


\section{NMR Spectra of Process Intermediates and Impurities}

${ }^{1} \mathrm{H}$ spectrum of $7 \mathrm{a}$ (DMSO- $d_{6}, 599 \mathrm{MHz}$ )

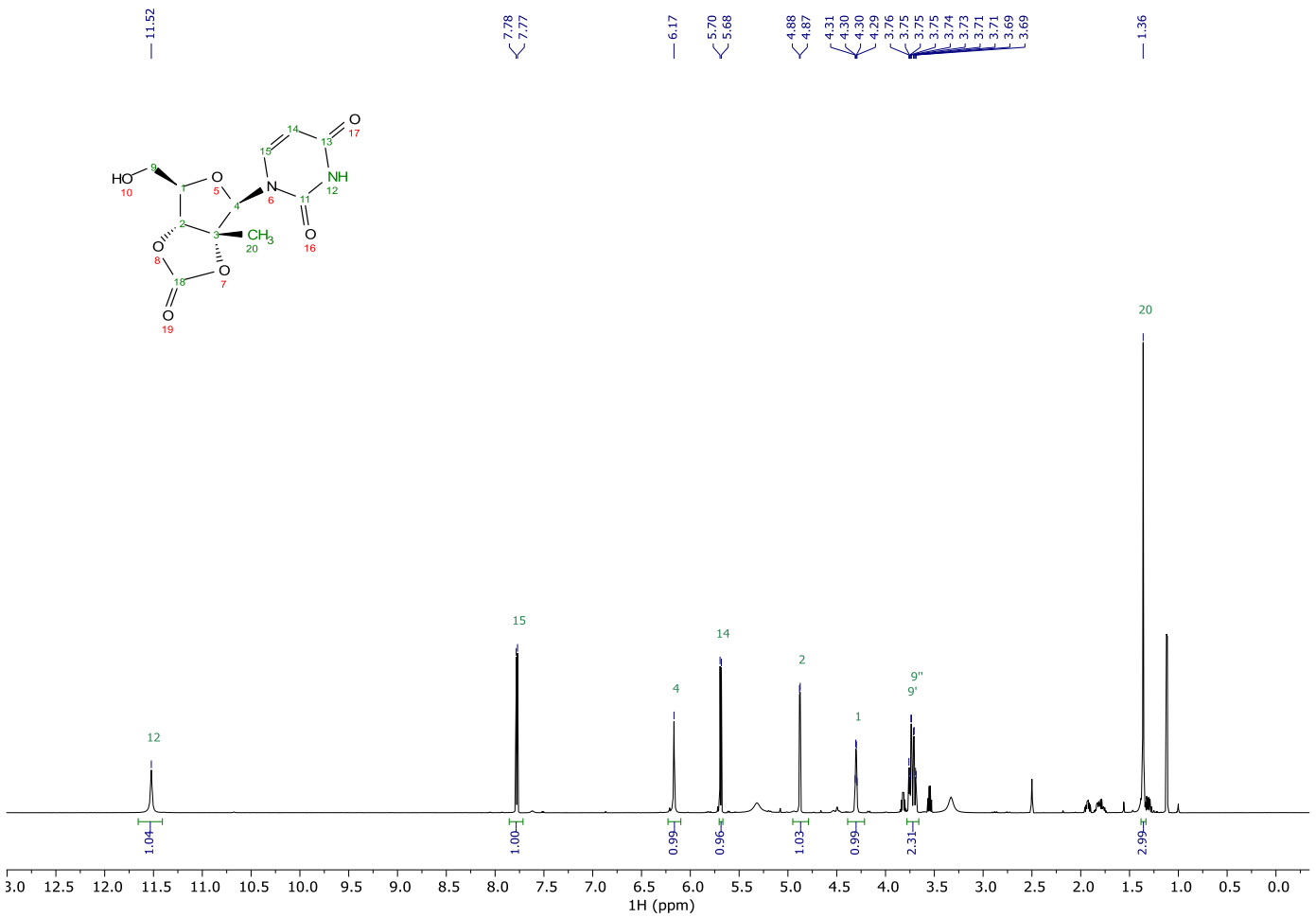

${ }^{13} \mathrm{C}\left\{{ }^{1} \mathrm{H}\right\}$ spectrum of 7a (DMSO- $d_{6}, 151 \mathrm{MHz}$ )

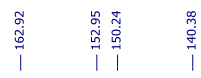

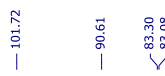

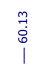

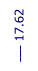
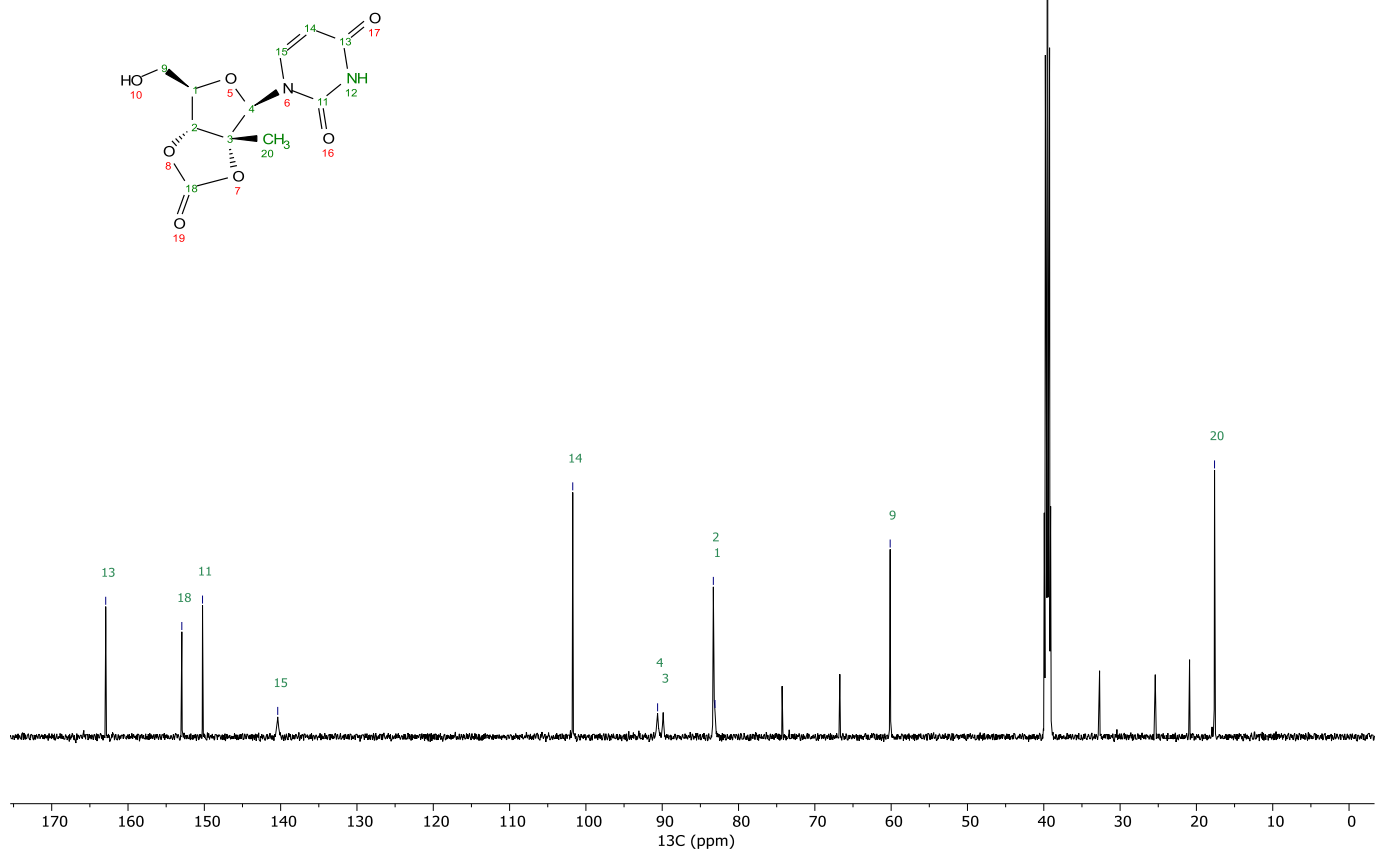
Hyde et al.

Supporting Information

${ }^{1} \mathrm{H}-{ }^{1} \mathrm{H}$ COSY spectrum of 7a (DMSO- $d_{6}, 599 \mathrm{MHz}$ )

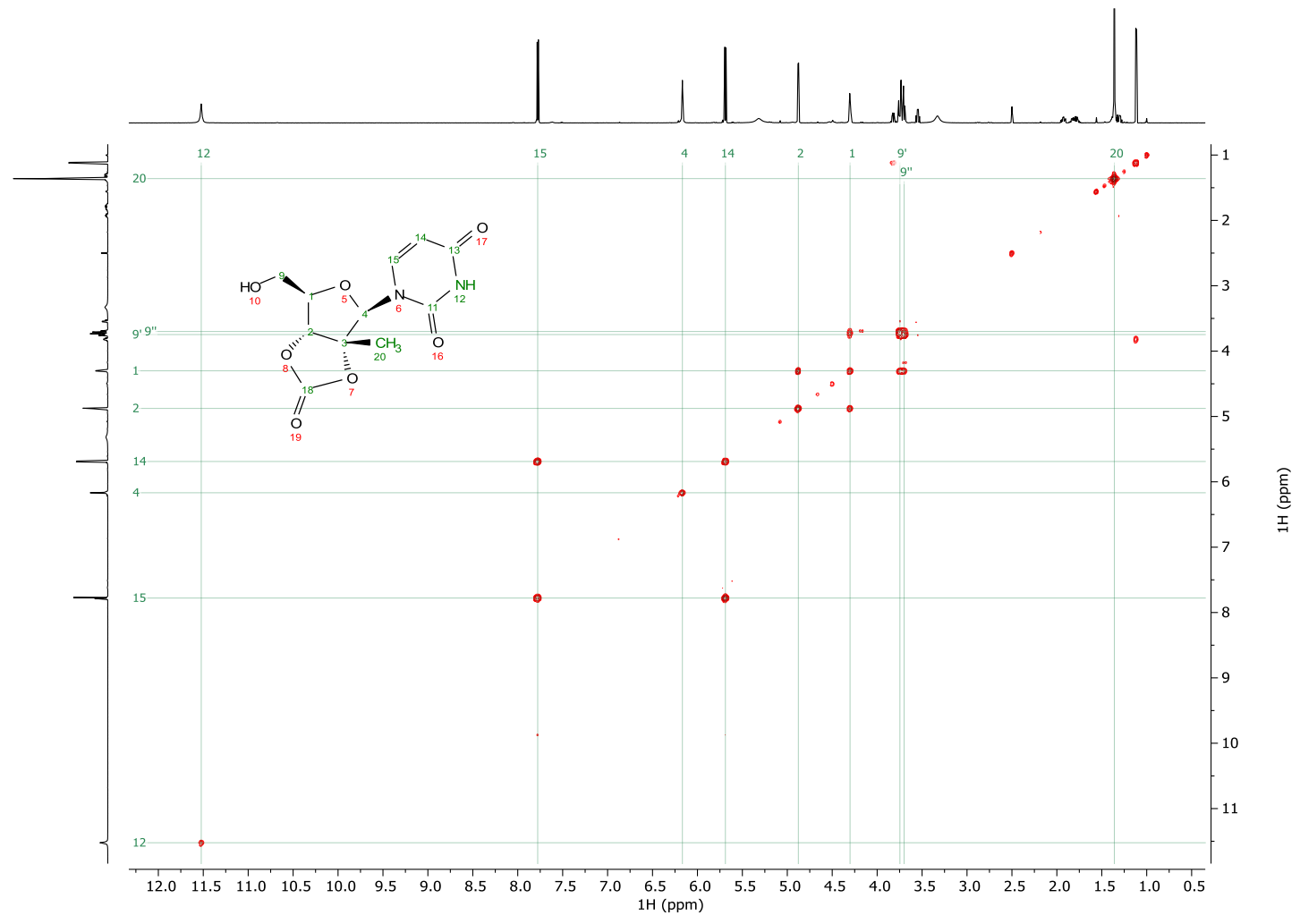

${ }^{1} \mathrm{H}-{ }^{13} \mathrm{C}$ HSQC spectrum of $4 a$ (DMSO- $d_{6}, 599 \mathrm{MHz}$ )

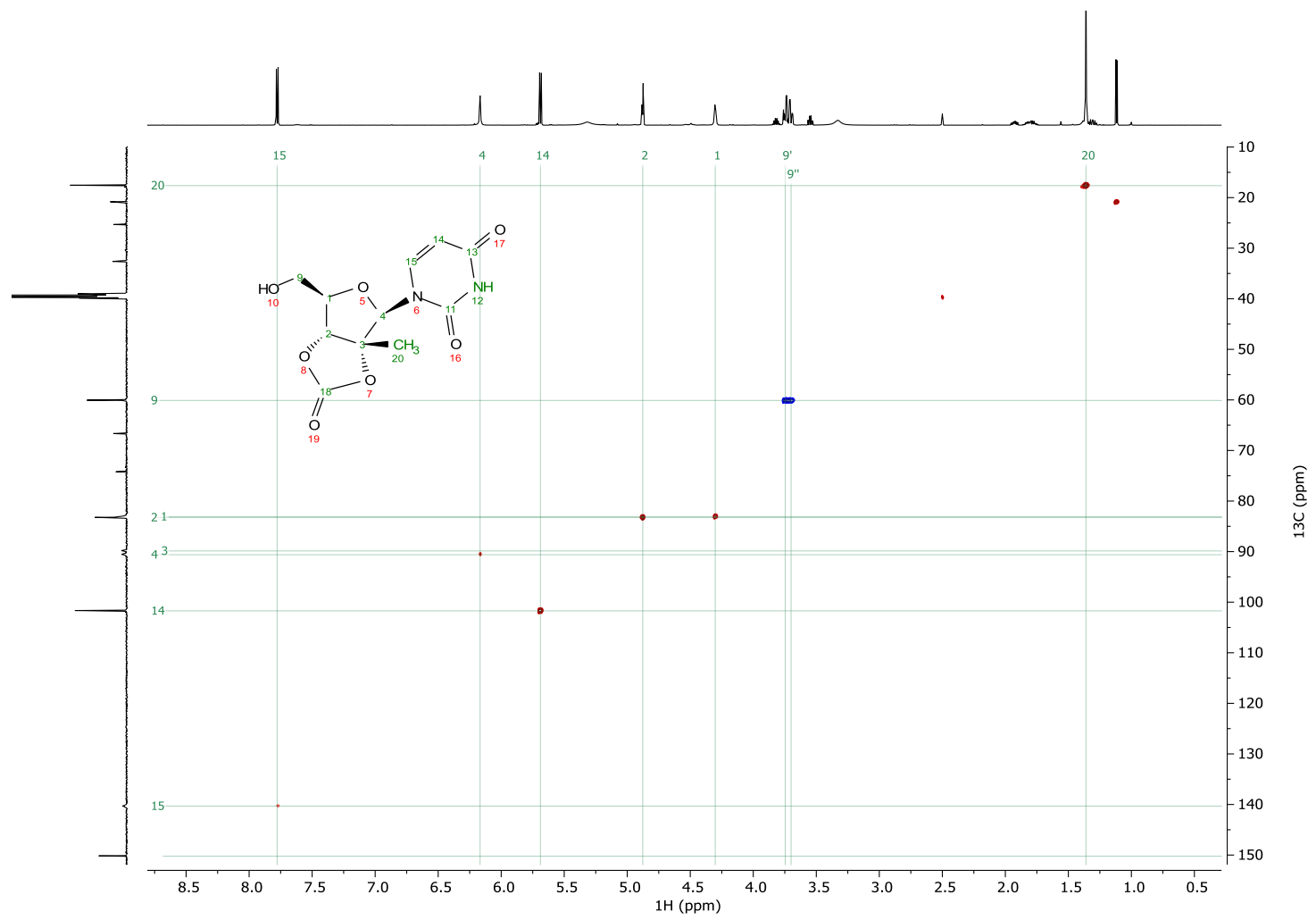


${ }^{1} \mathrm{H}-{ }^{13} \mathrm{C} \mathrm{HMBC}$ spectrum of 7a (DMSO- $d_{6}, 599 \mathrm{MHz}$ )

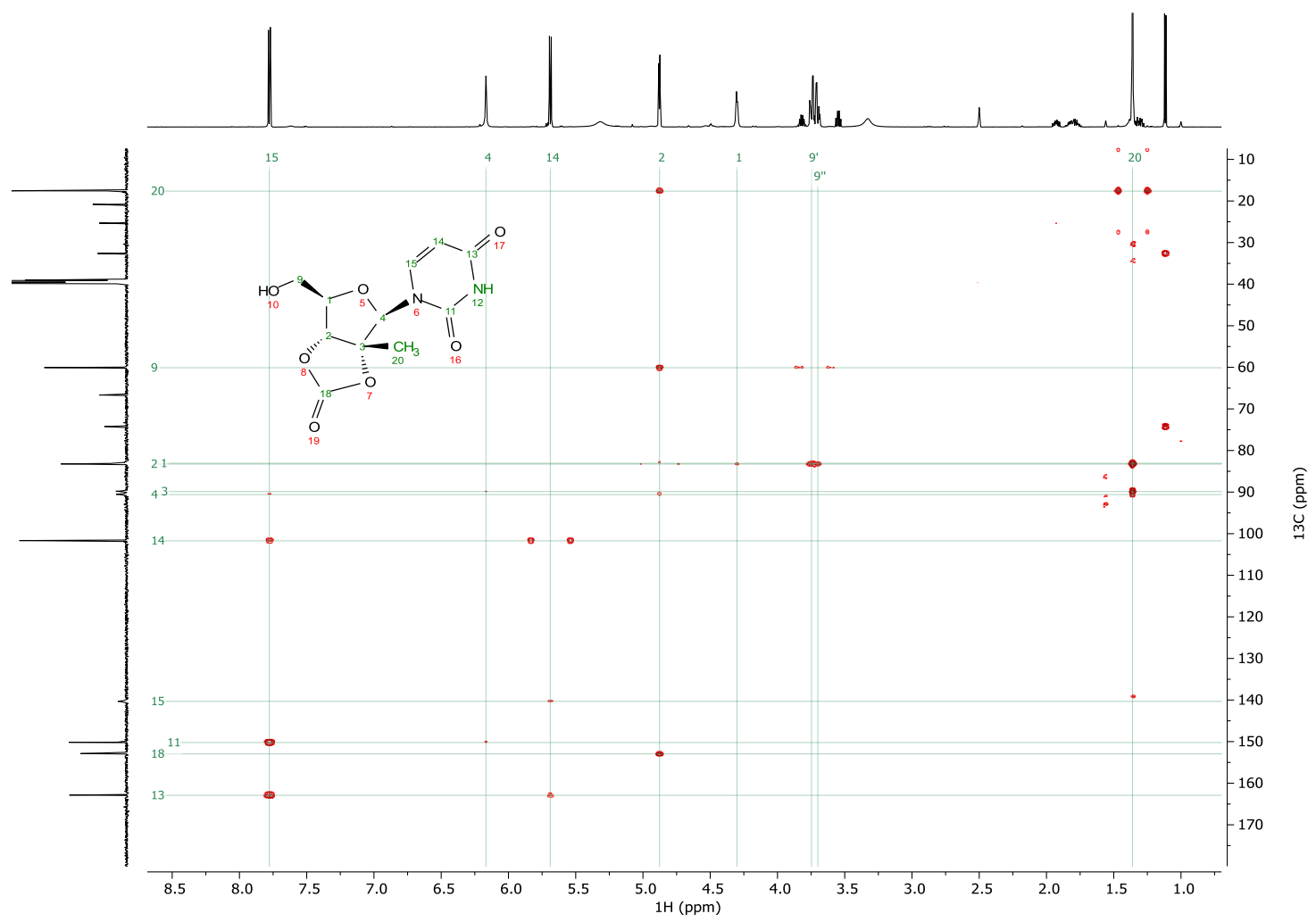

${ }^{1} \mathrm{H}-{ }^{1} \mathrm{H}$ NOESY spectrum of $7 \mathrm{a}$ (DMSO- $d_{6}, 599 \mathrm{MHz}$ )

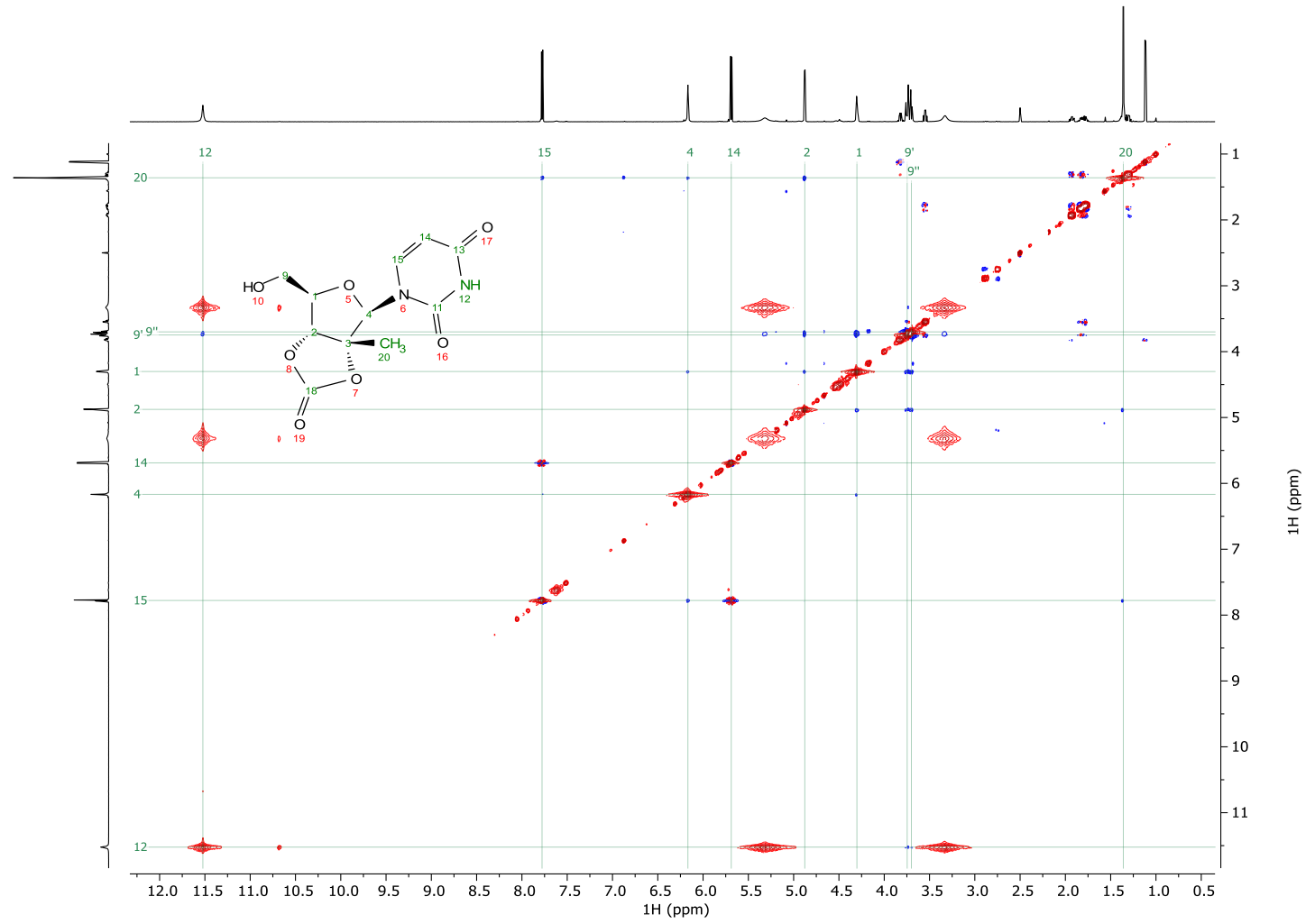


${ }^{1} \mathrm{H}$ spectrum of $7 \mathbf{b}$ (DMSO- $d_{6}, 600 \mathrm{MHz}$ )

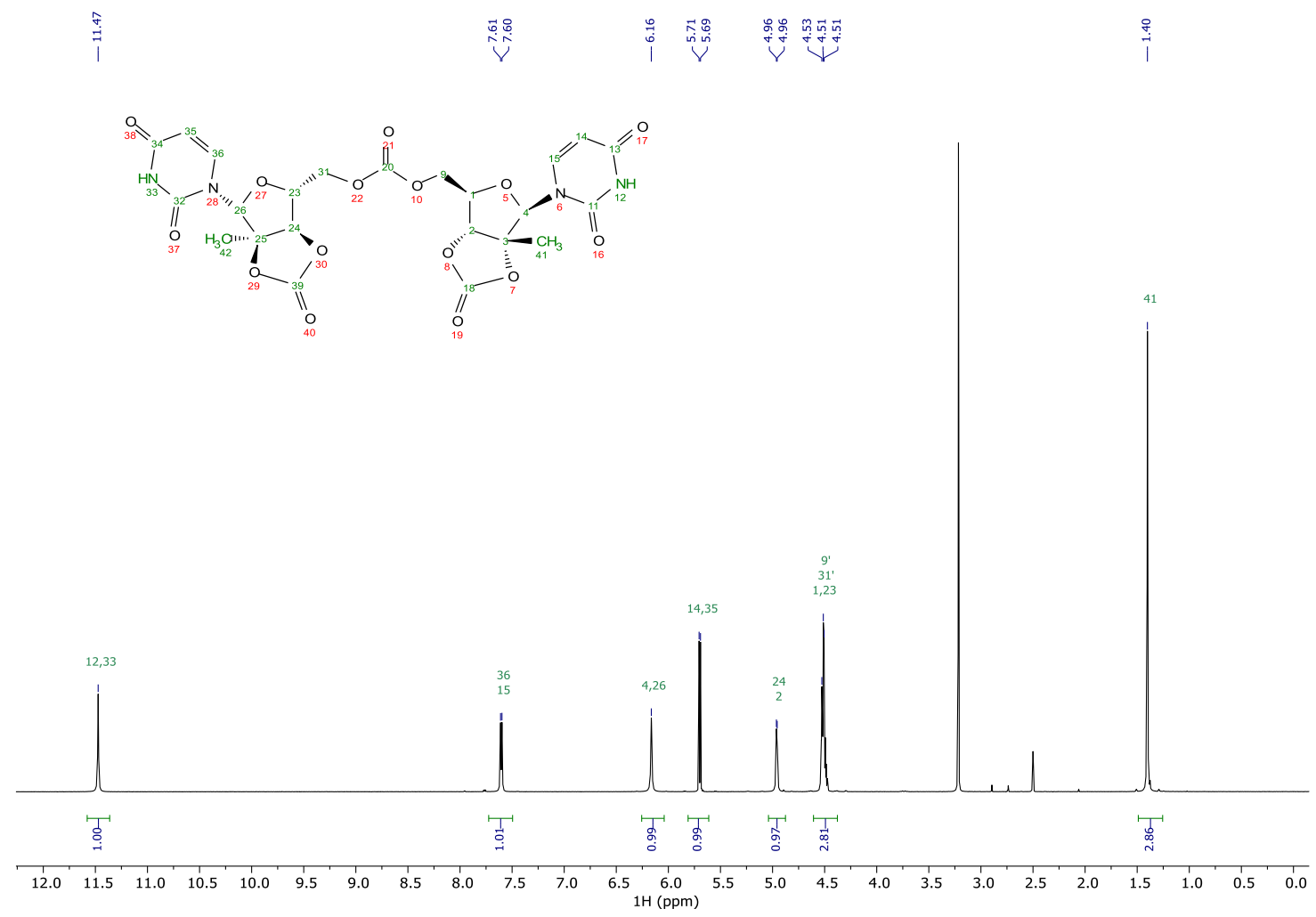

${ }^{13} \mathrm{C}\left\{{ }^{1} \mathrm{H}\right\}$ spectrum of $7 \mathrm{~b}$ (DMSO- $d_{6}, 151 \mathrm{MHz}$ )
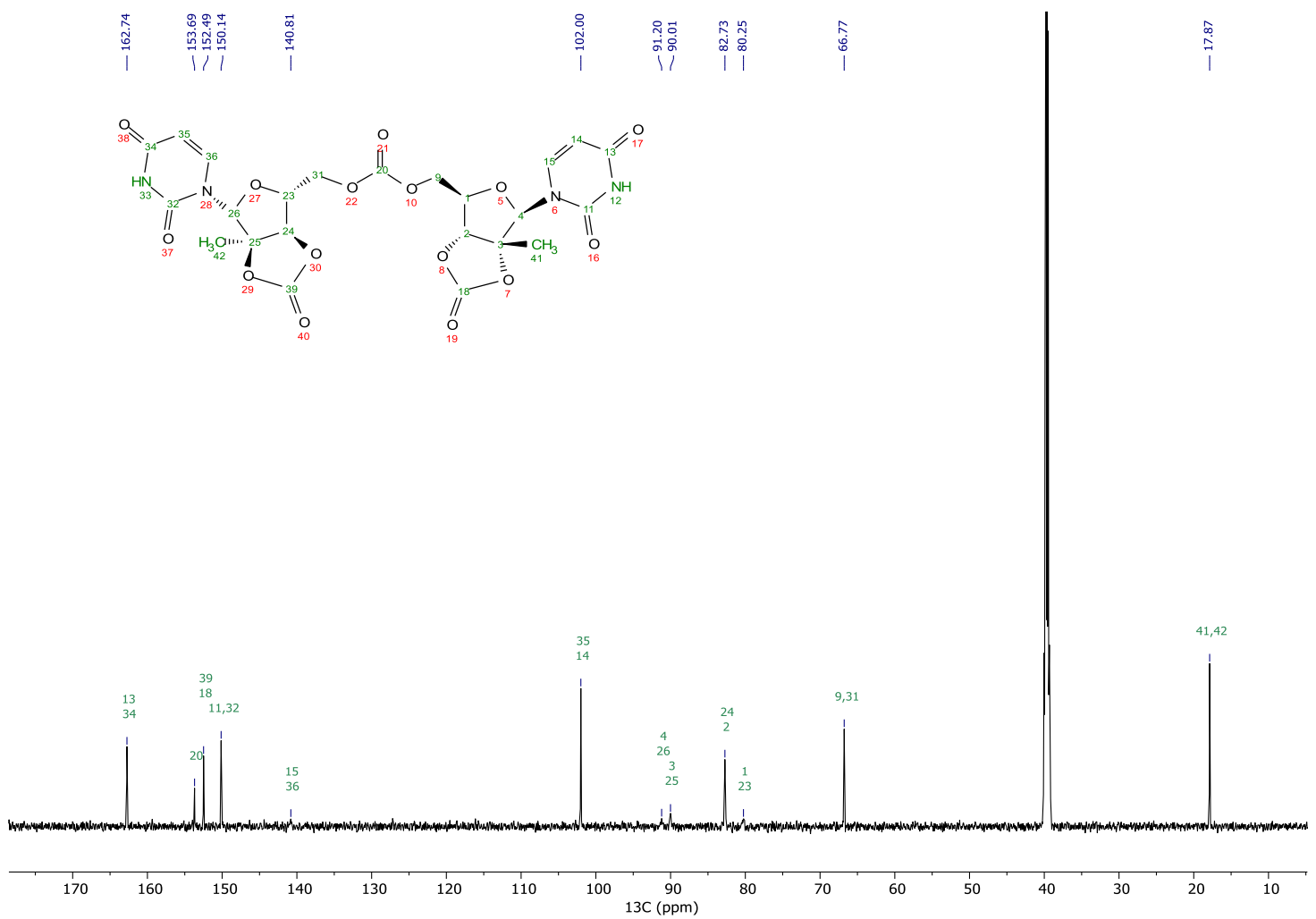
${ }^{1} \mathrm{H}-{ }^{1} \mathrm{H}$ COSY spectrum of $7 \mathrm{~b}$ (DMSO- $d_{6}, 600 \mathrm{MHz}$ )

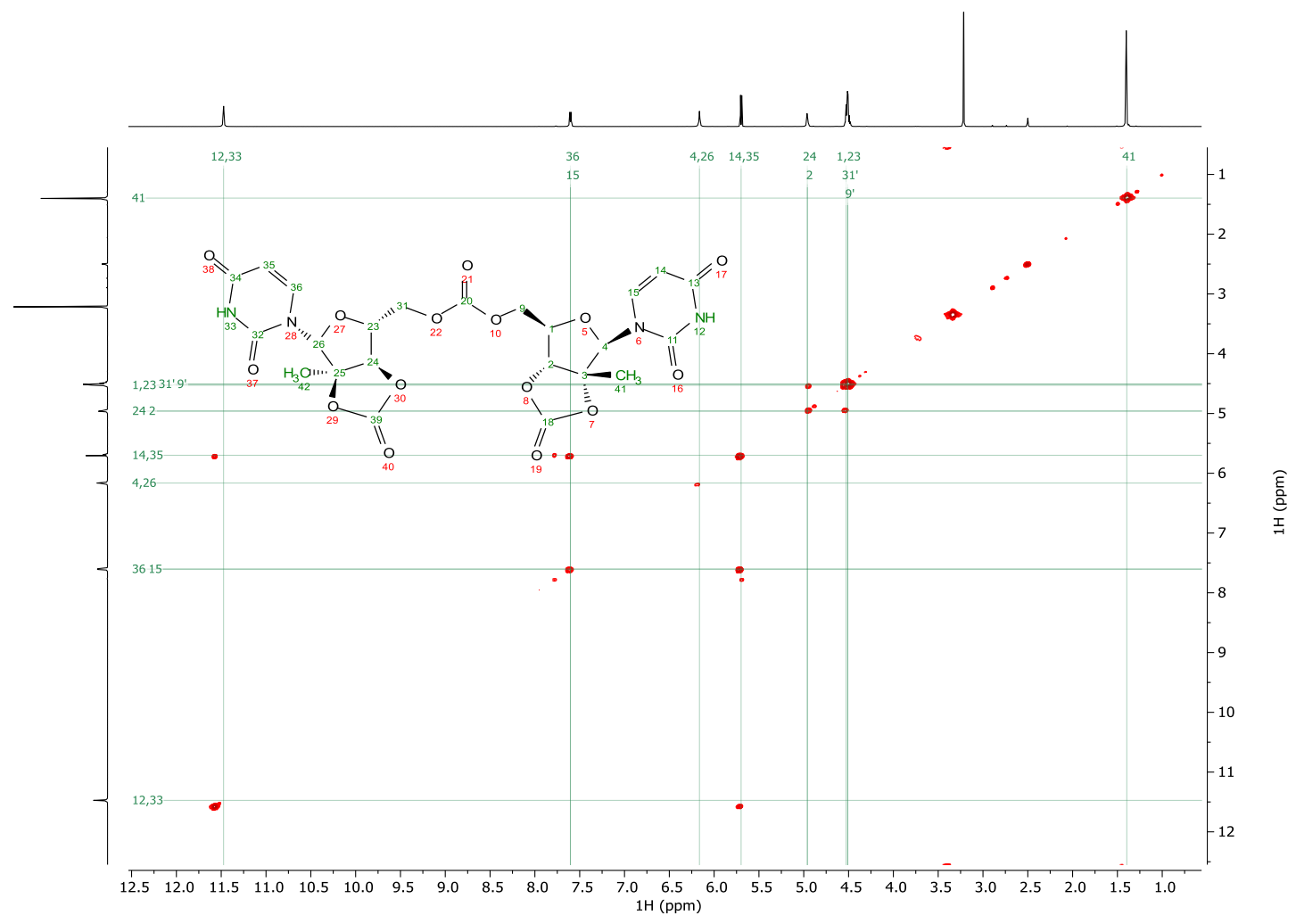

${ }^{1} \mathrm{H}-{ }^{13} \mathrm{C}$ HSQC spectrum of 7b (DMSO- $d_{6}, 600 \mathrm{MHz}$ )

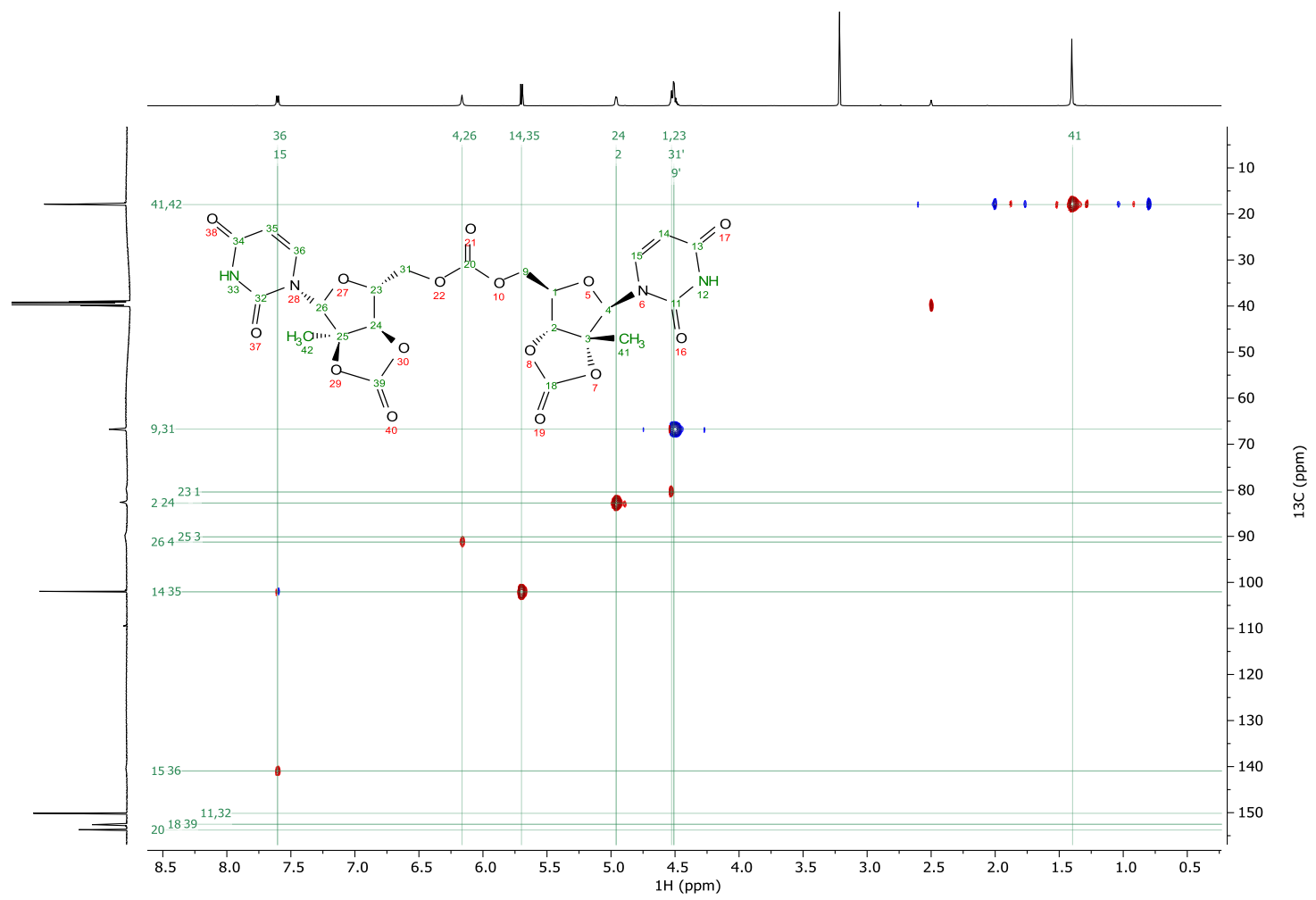


${ }^{1} \mathrm{H}-{ }^{13} \mathrm{C} \mathrm{HMBC}$ spectrum of $7 \mathrm{~b}$ (DMSO- $d_{6}, 600 \mathrm{MHz}$ )

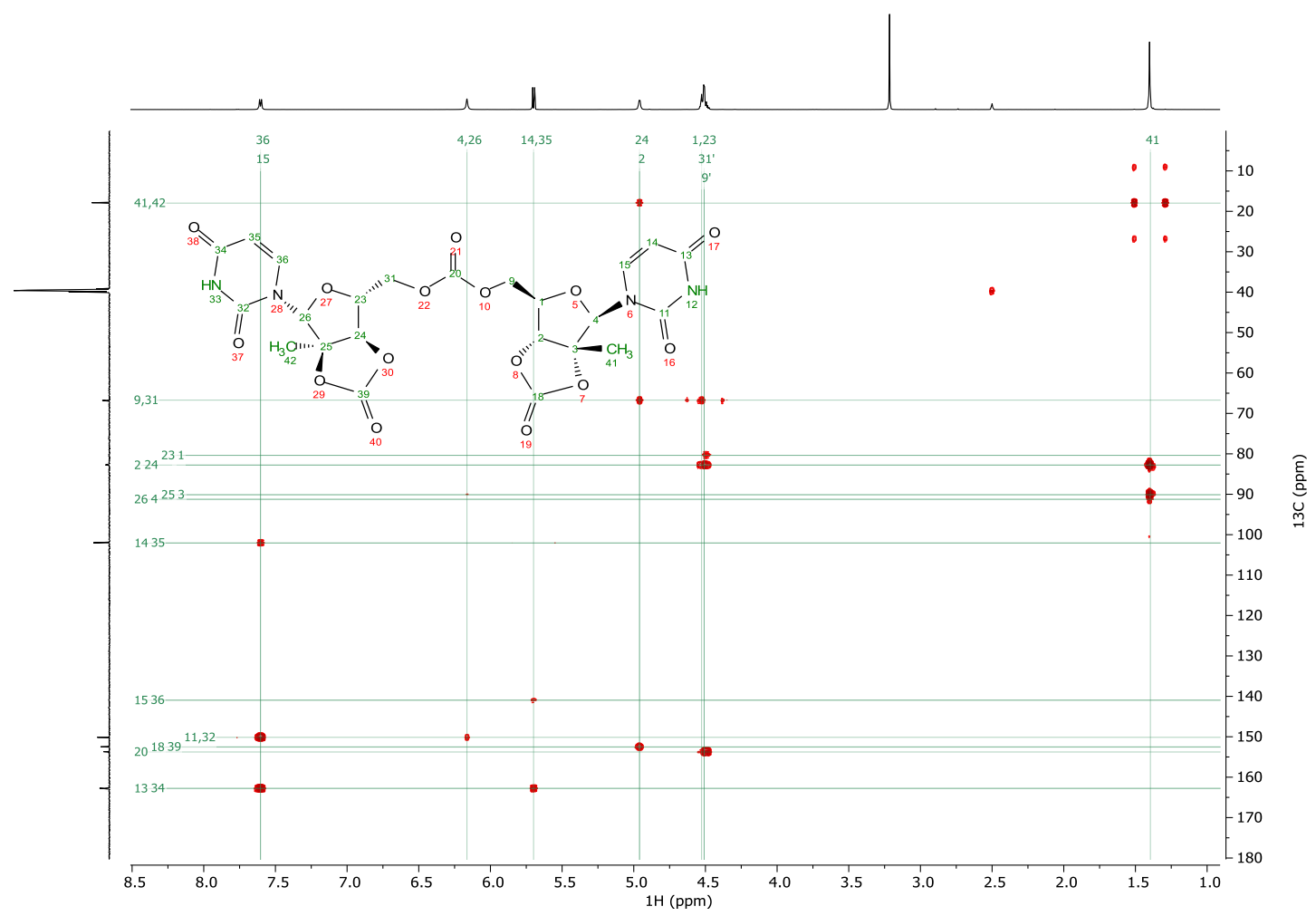

${ }^{1} \mathrm{H}-{ }^{1} \mathrm{H}$ NOESY spectrum of $7 \mathbf{b}$ (DMSO- $d_{6}, 600 \mathrm{MHz}$ )

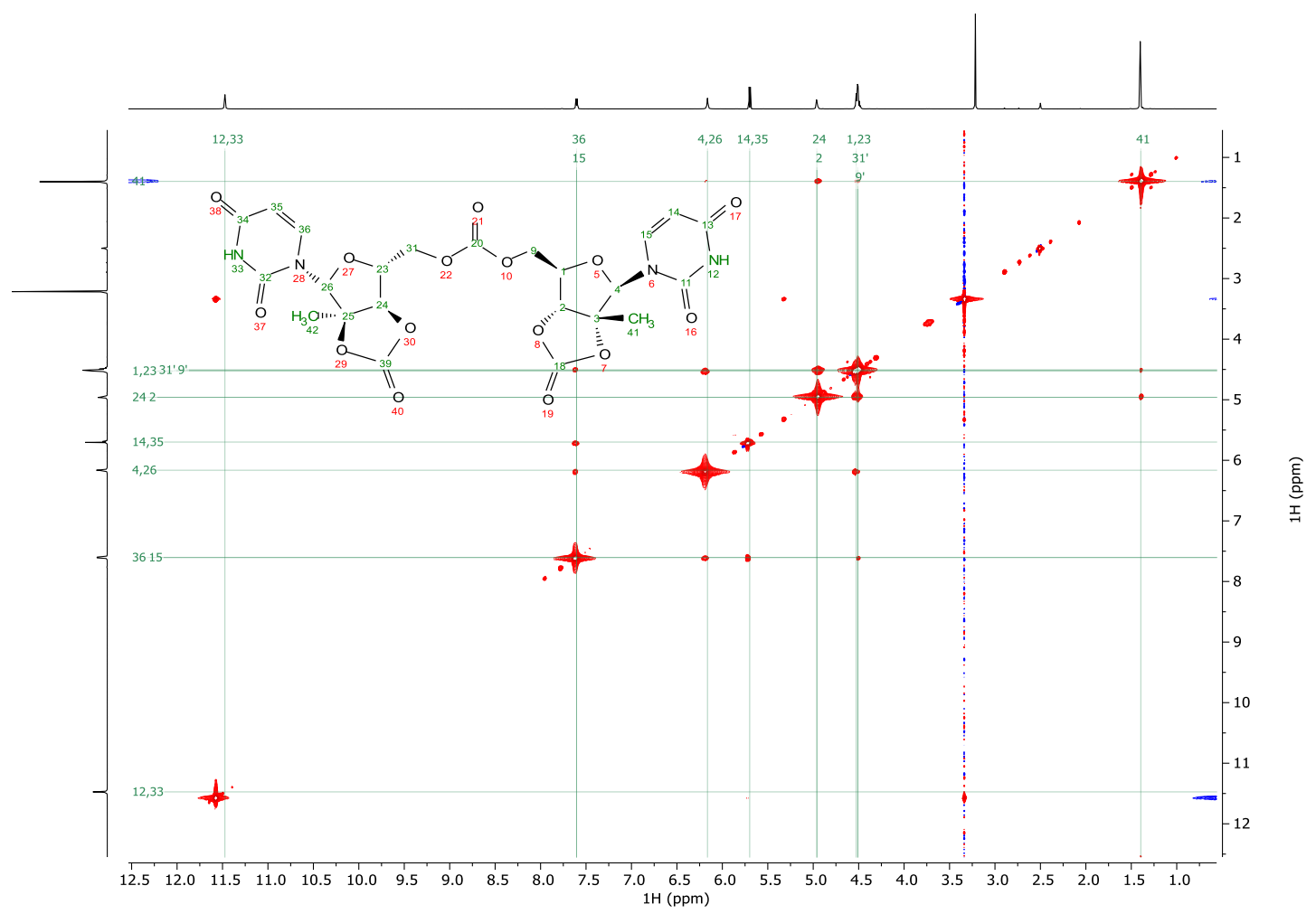


${ }^{1} \mathrm{H}$ spectrum of $8 \mathrm{a}$ (DMSO- $d_{6}, 599 \mathrm{MHz}$ )<smiles>C1=CCCCC1</smiles>

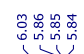

产<smiles>CC12Oc3nc(=O)ncn3C1OC(CO)C2O</smiles>

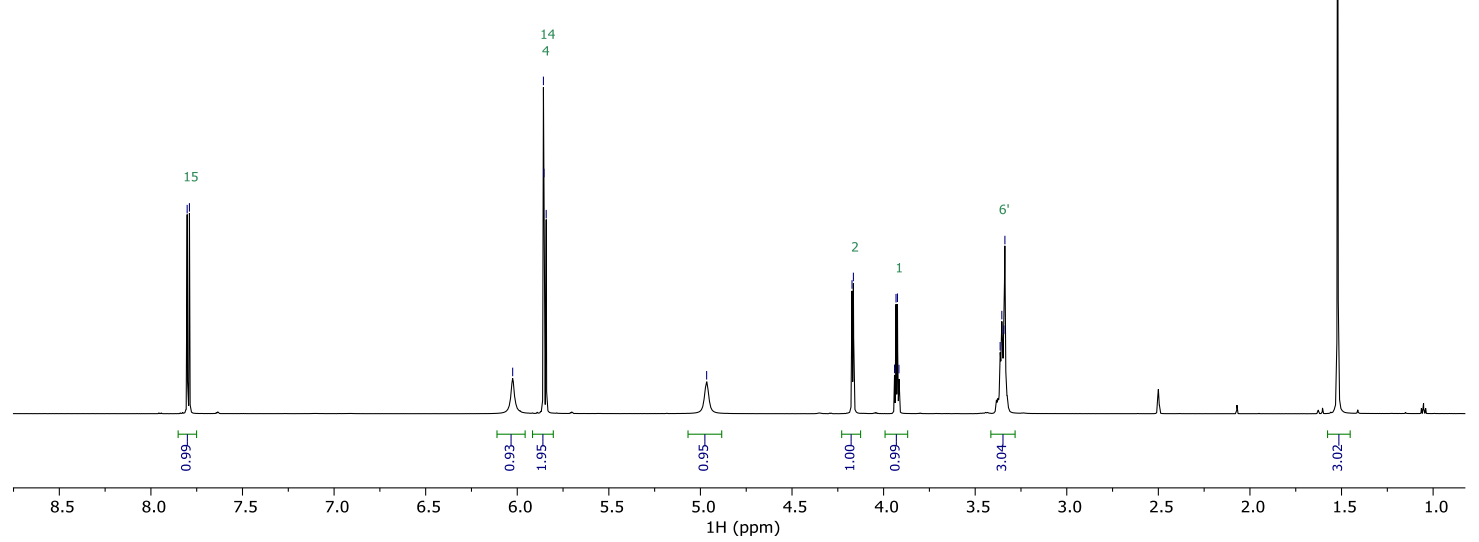

${ }^{13} \mathrm{C}\left\{{ }^{1} \mathrm{H}\right\}$ spectrum of $8 \mathbf{a}$ (DMSO- $d_{6}, 151 \mathrm{MHz}$ )
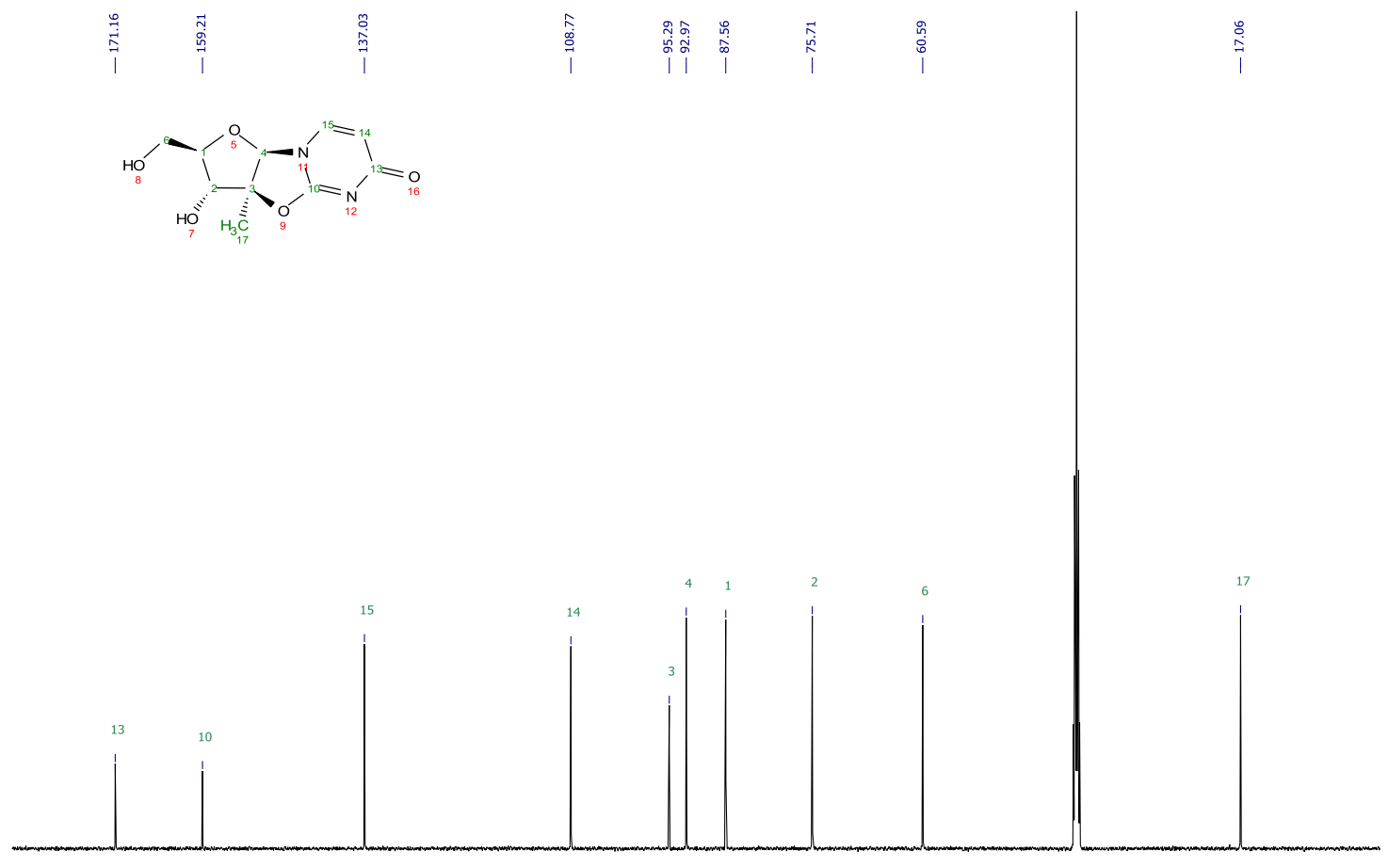

180

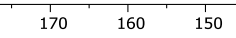

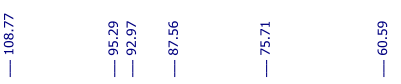

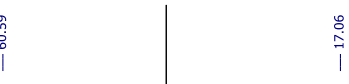


${ }^{1} \mathrm{H}-{ }^{13} \mathrm{C}$ HSQC spectrum of $8 \mathrm{a}$ (DMSO- $d_{6}, 599 \mathrm{MHz}$ )

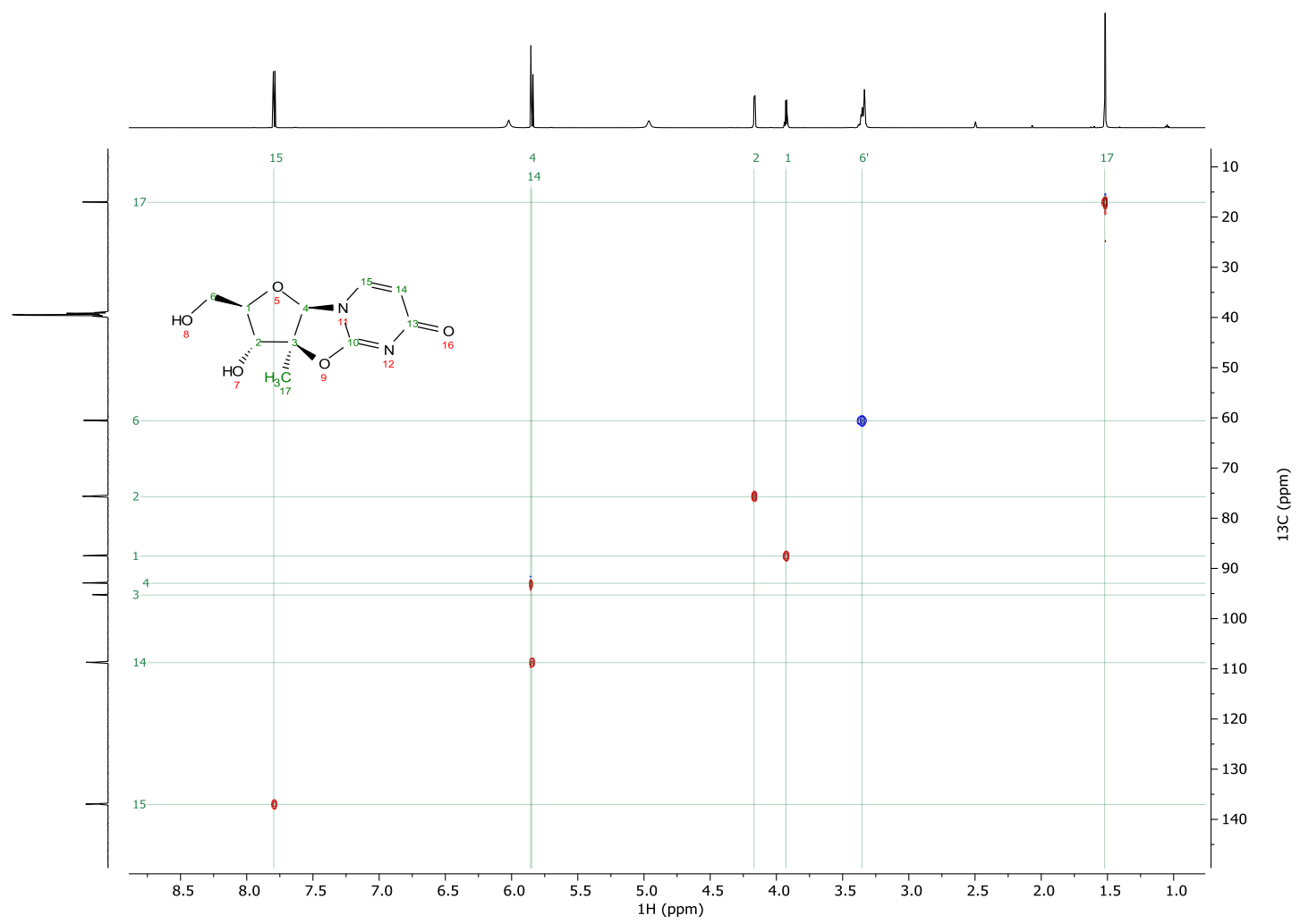

${ }^{1} \mathrm{H}-{ }^{13} \mathrm{C}$ HMBC spectrum of 8a (DMSO- $d_{6}, 599 \mathrm{MHz}$ )

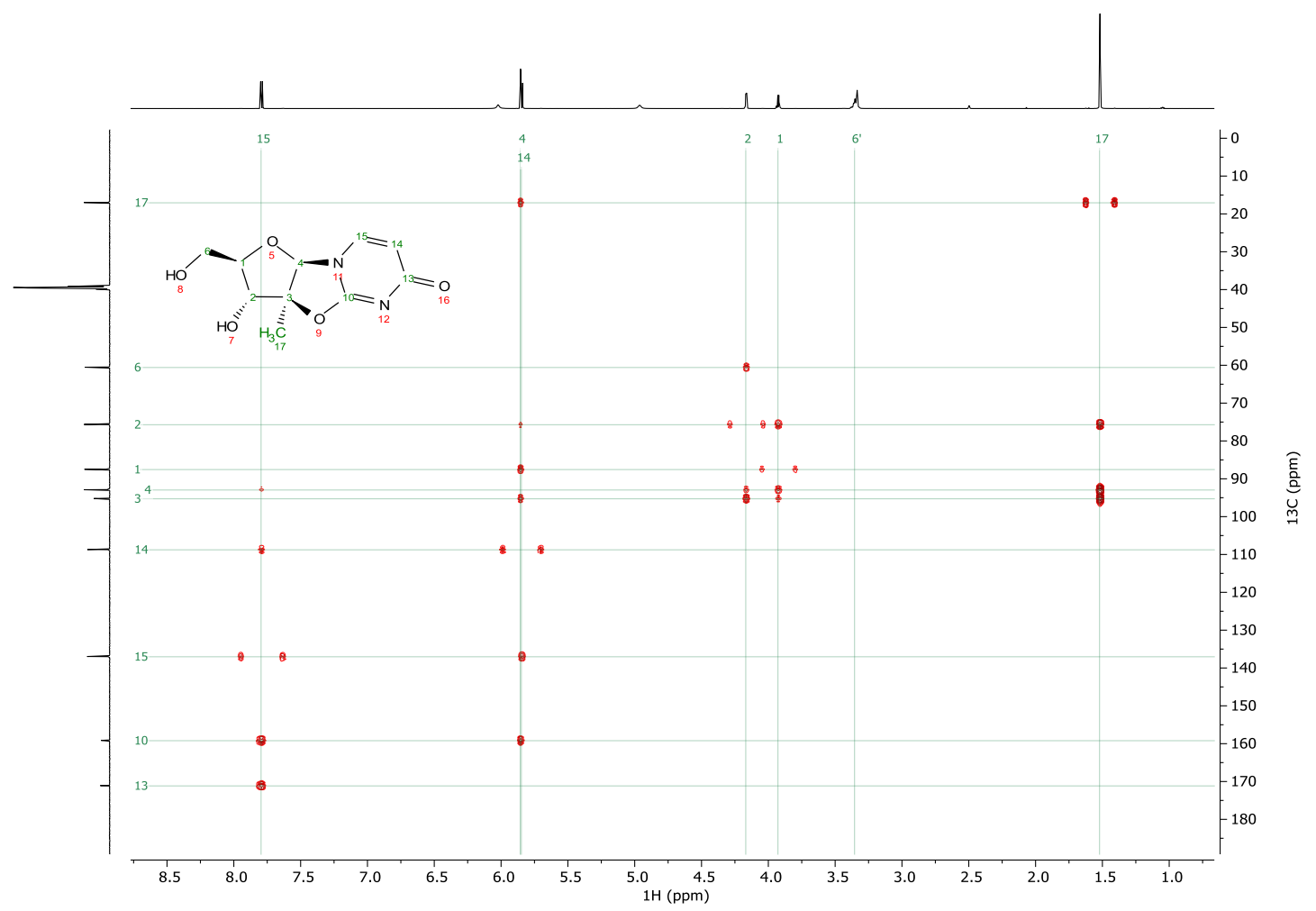


${ }^{1} \mathrm{H}-{ }^{1} \mathrm{H}$ NOESY spectrum of $8 \mathrm{a}$ (DMSO- $d_{6}, 599 \mathrm{MHz}$ )

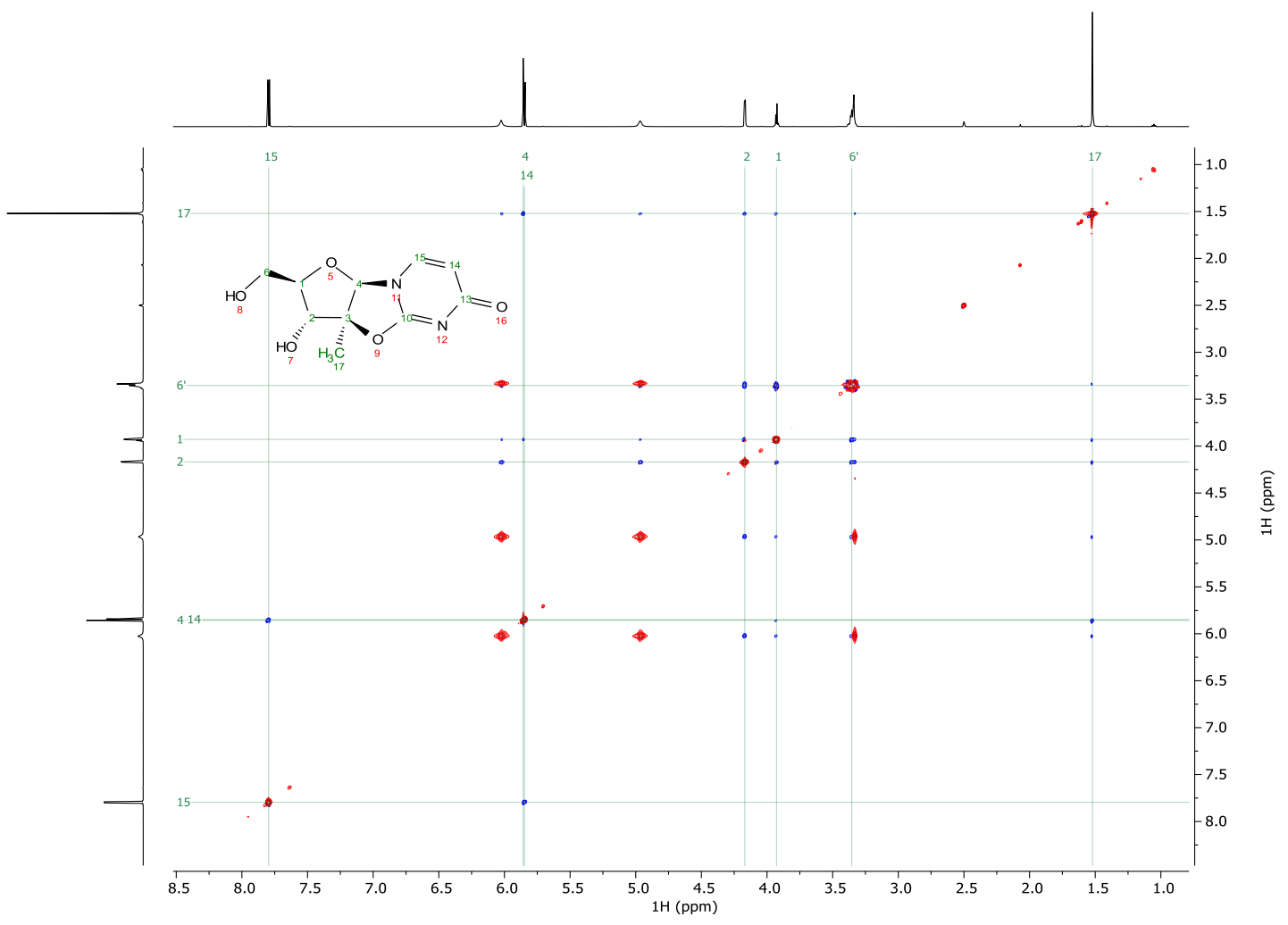

${ }^{1} \mathrm{H}$ spectrum of $\mathbf{8 b}\left(\mathrm{CD}_{3} \mathrm{CN}+\mathrm{DMSO}-d_{6}, 600 \mathrm{MHz}\right)$
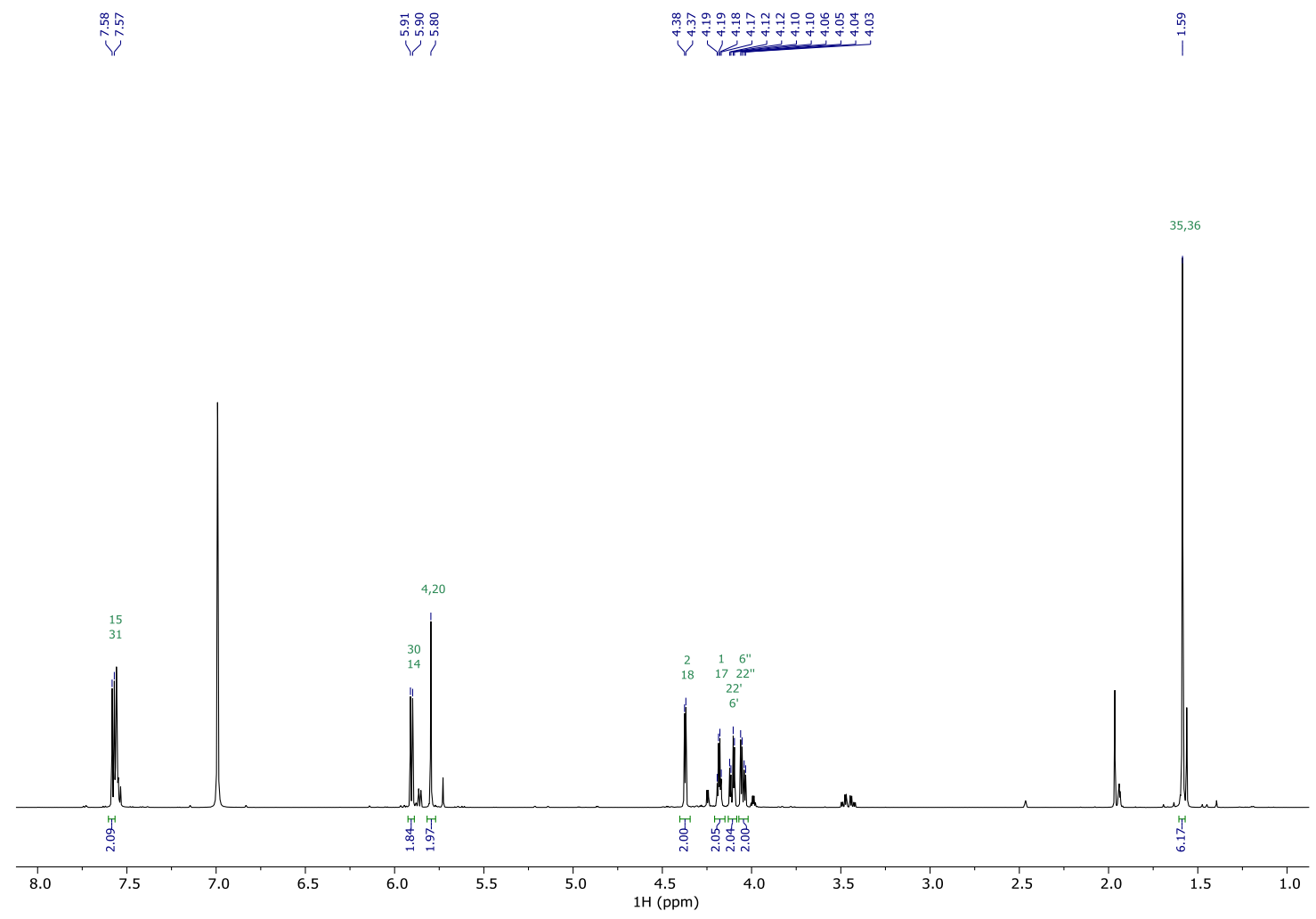
${ }^{13} \mathrm{C}\left\{{ }^{1} \mathrm{H}\right\}$ spectrum of $\mathbf{8 b}\left(\mathrm{CD}_{3} \mathrm{CN}+\mathrm{DMSO}-\mathrm{d}_{6}, 151 \mathrm{MHz}\right)$

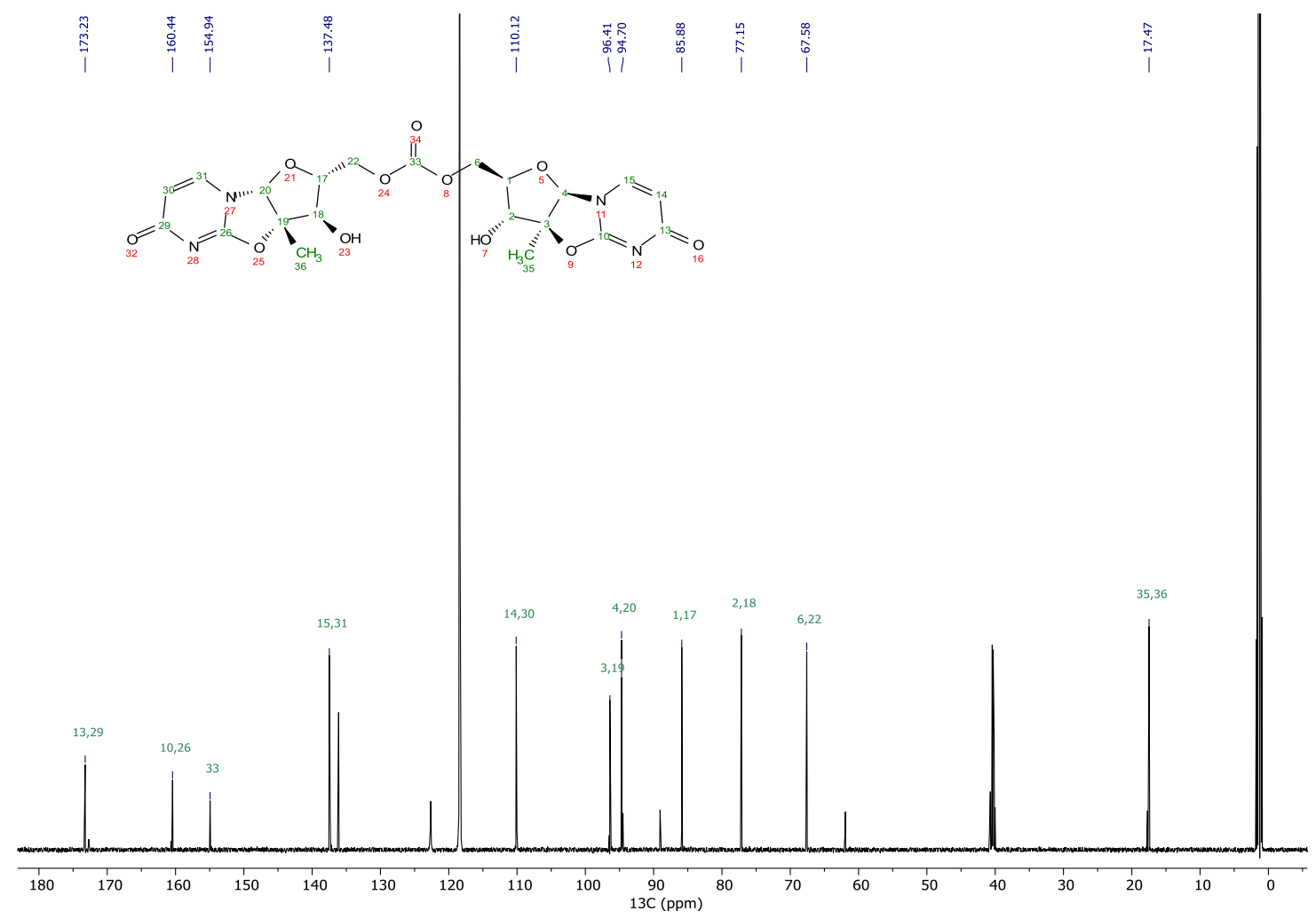

${ }^{1} \mathrm{H}-{ }^{1} \mathrm{H}$ COSY spectrum of $\mathbf{8 b}\left(\mathrm{CD}_{3} \mathrm{CN}+\mathrm{DMSO}-d_{6}, 600 \mathrm{MHz}\right)$

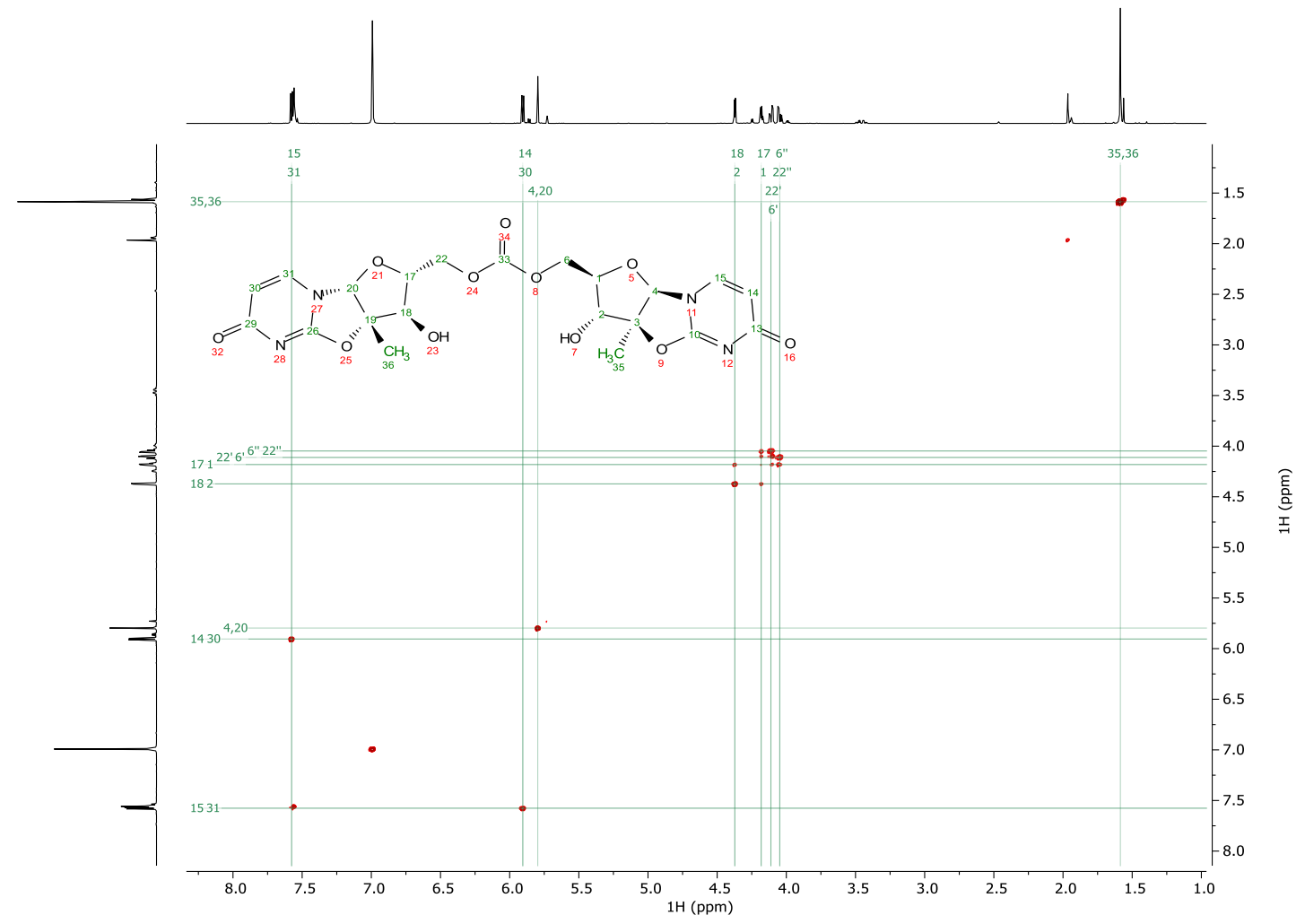


${ }^{1} \mathrm{H}-{ }^{13} \mathrm{C}$ HSQC spectrum of $\mathbf{8 b}\left(\mathrm{CD}_{3} \mathrm{CN}+\right.$ DMSO- $\left.d_{6}, 600 \mathrm{MHz}\right)$

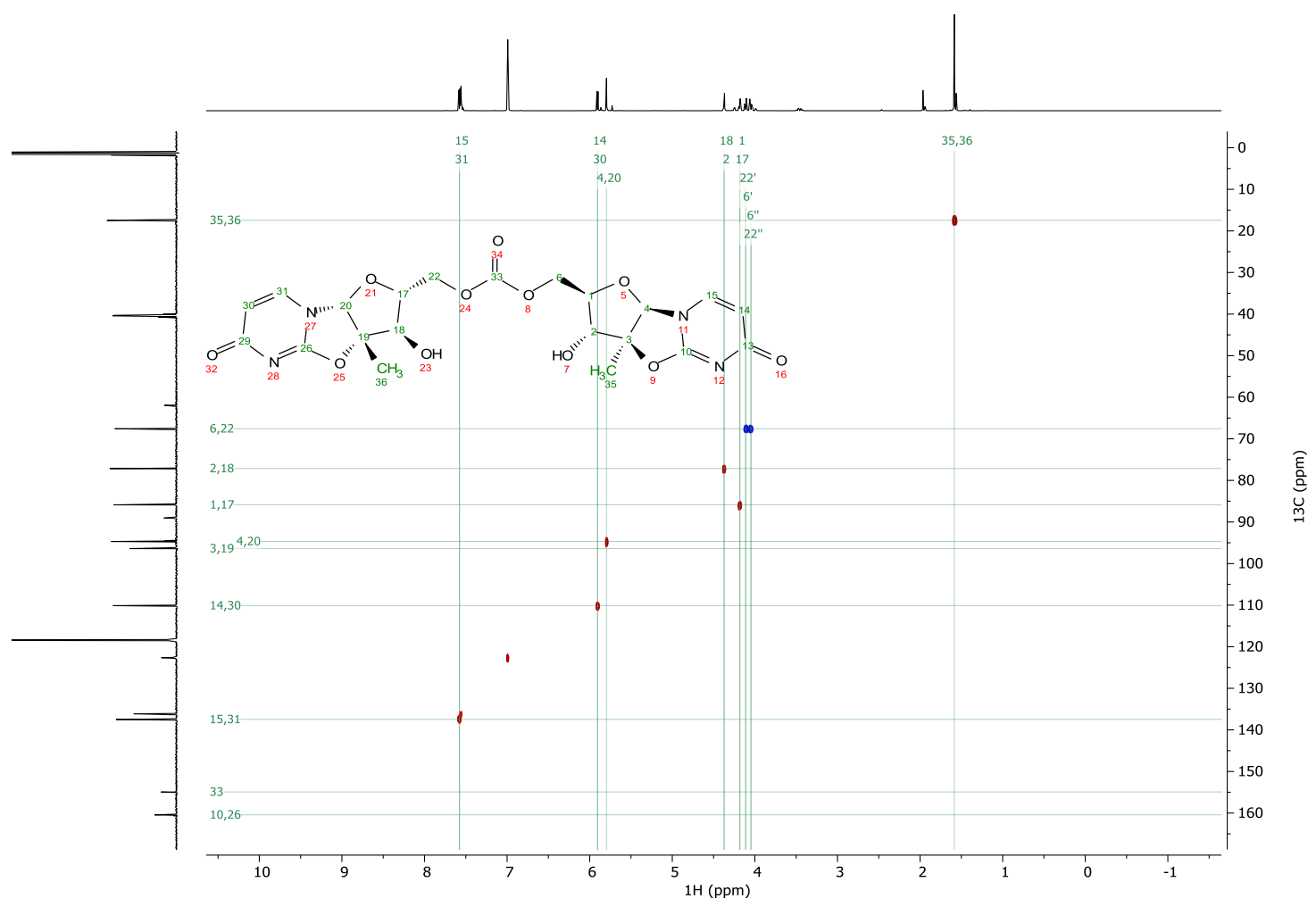

${ }^{1} \mathrm{H}-{ }^{13} \mathrm{C}$ HMBC spectrum of $8 \mathbf{b}\left(\mathrm{CD}_{3} \mathrm{CN}+\right.$ DMSO- $\left.d_{6}, 600 \mathrm{MHz}\right)$

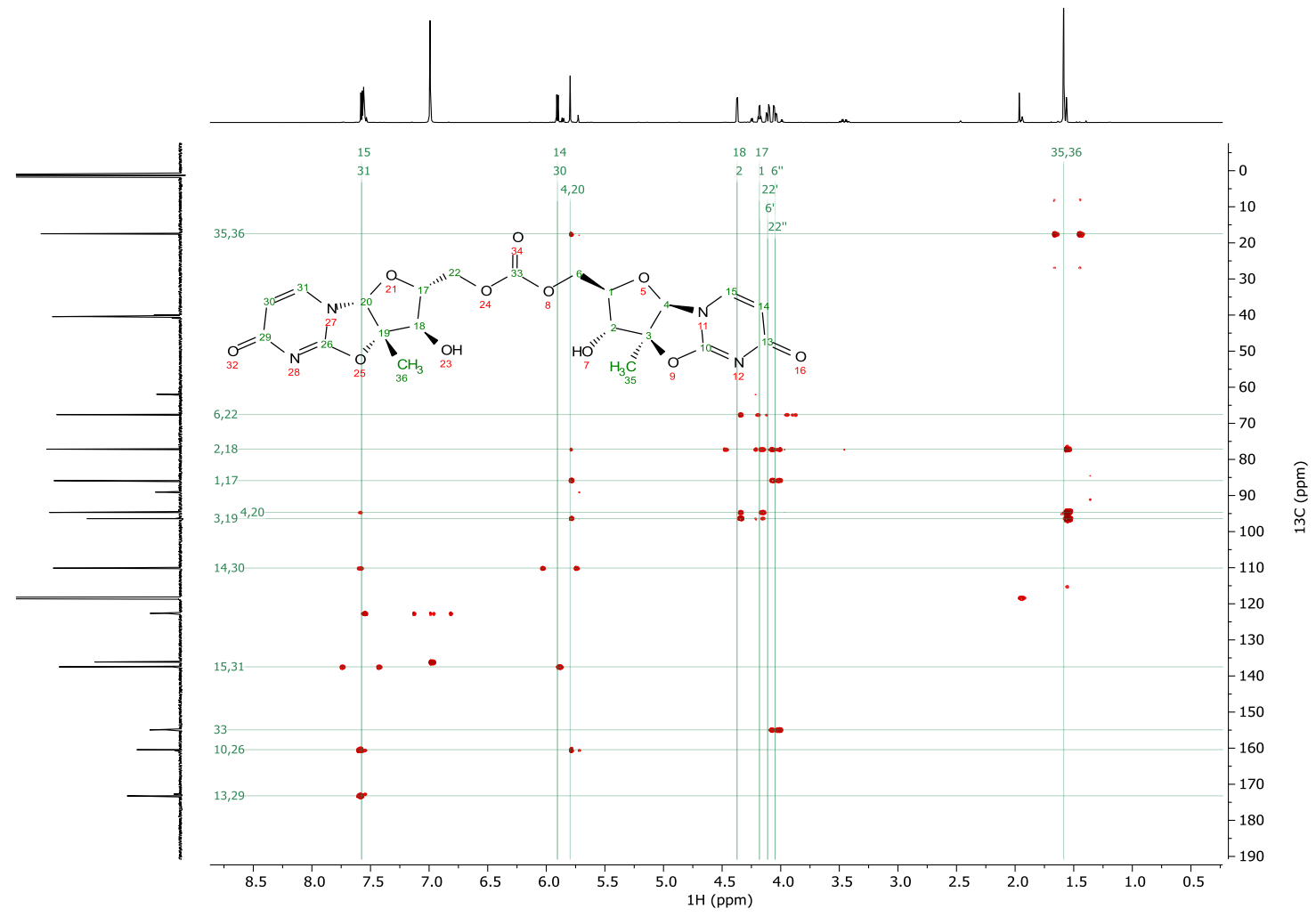


${ }^{1} \mathrm{H}-{ }^{1} \mathrm{H}$ NOESY spectrum of $\mathbf{8 b}\left(\mathrm{CD}_{3} \mathrm{CN}+\mathrm{DMSO}-d_{6}, 600 \mathrm{MHz}\right)$

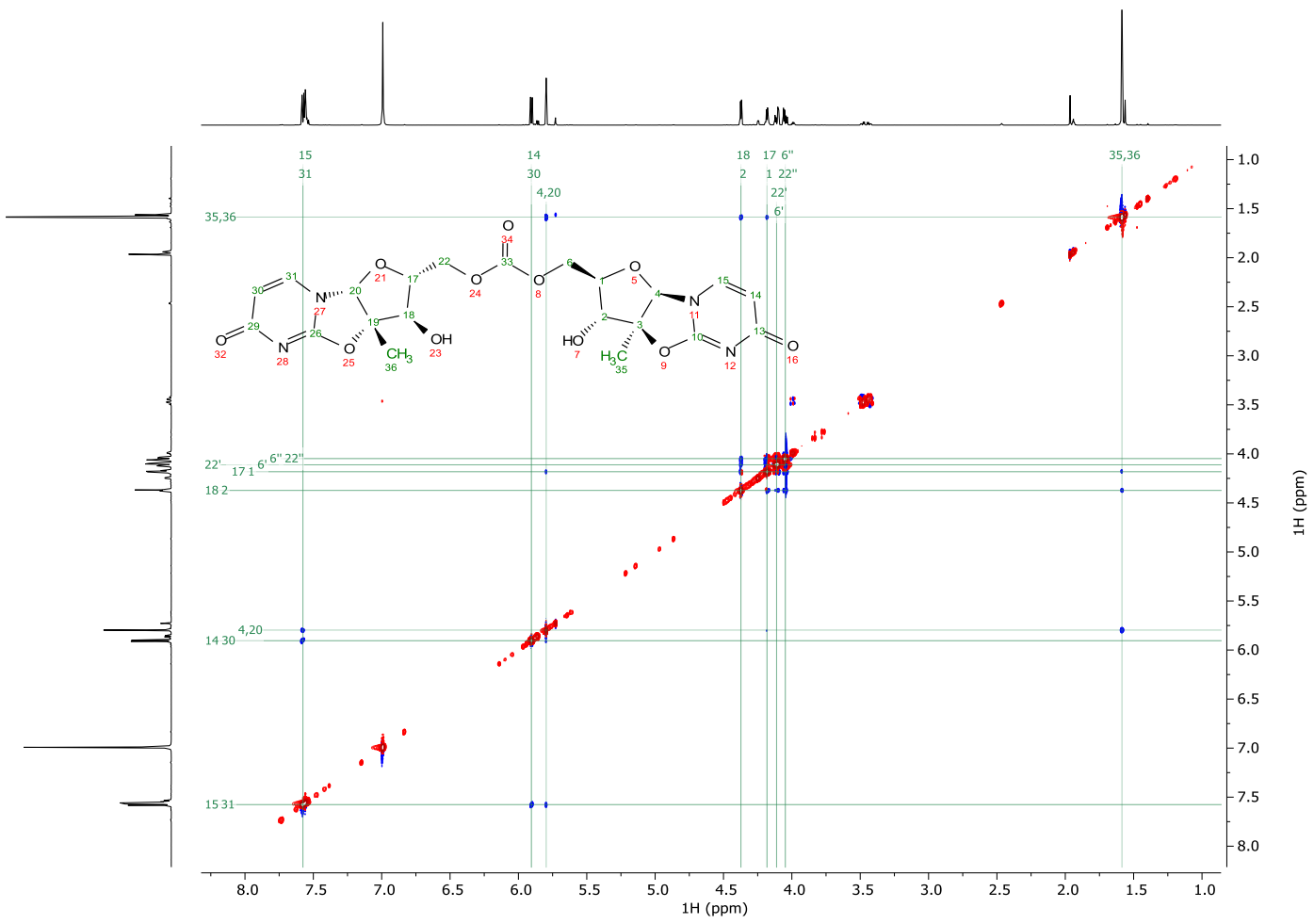

${ }^{1} \mathrm{H}$ spectrum of 9 (DMSO- $d_{6}, 600 \mathrm{MHz}$ )

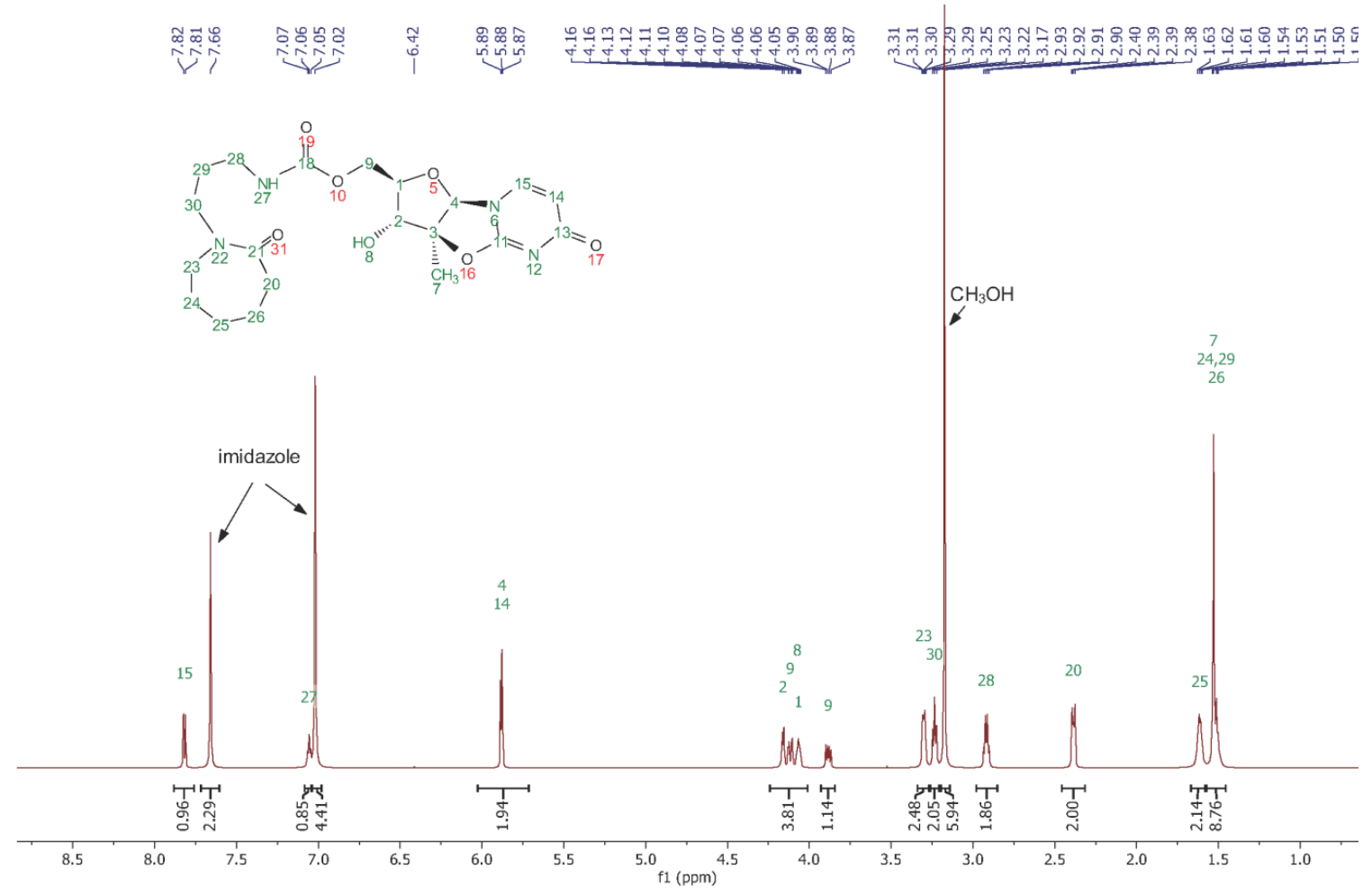


${ }^{13} \mathrm{C}\left\{{ }^{1} \mathrm{H}\right\}$ spectrum of 9 (DMSO- $d_{6}, 150 \mathrm{MHz}$ )

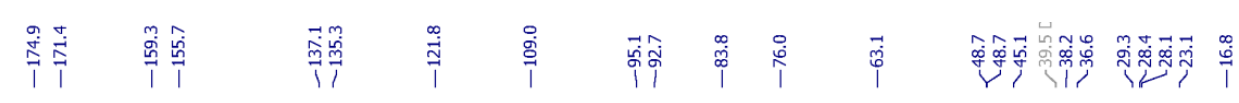
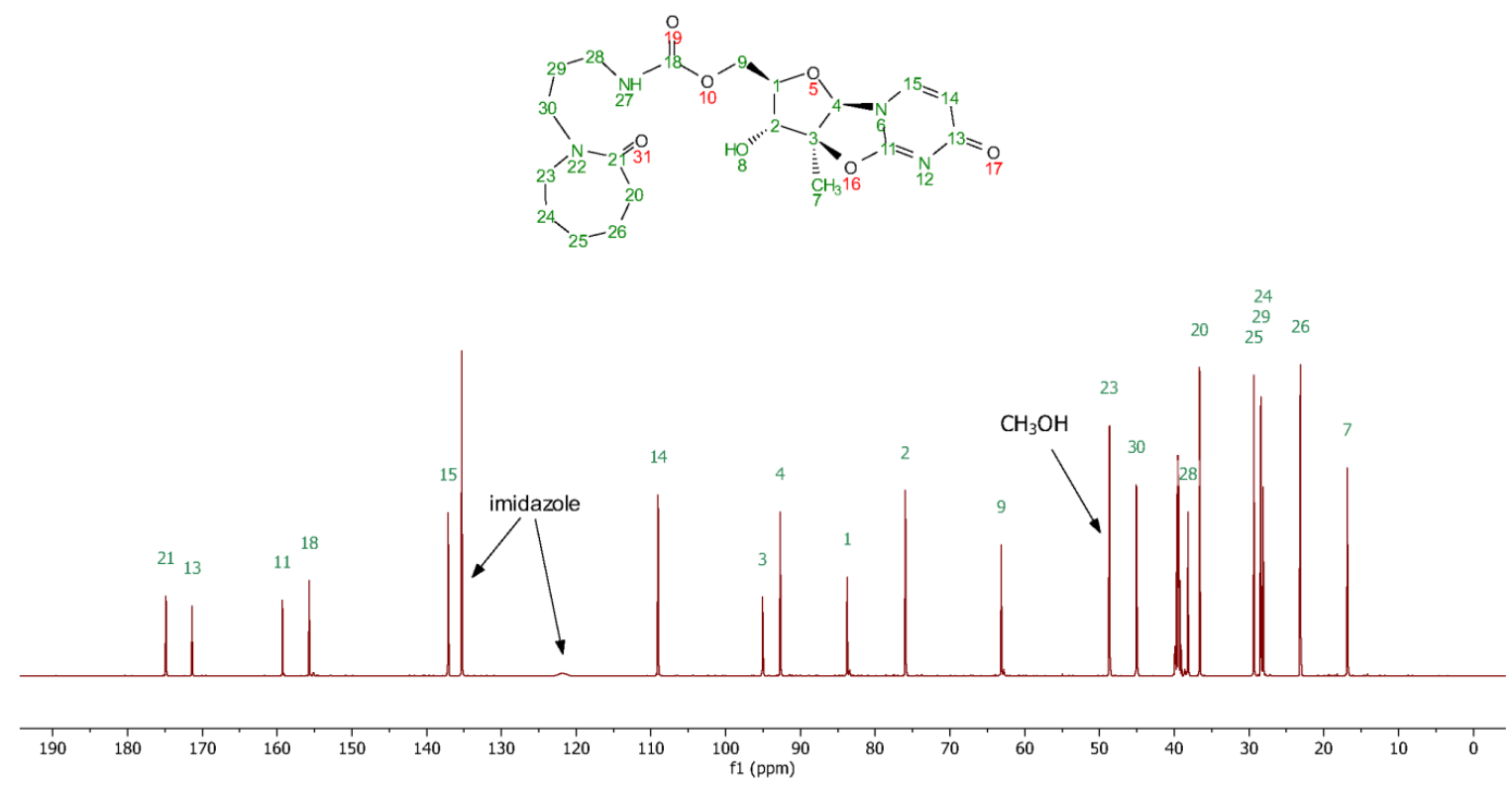

${ }^{1} \mathrm{H}-{ }^{1} \mathrm{H}$ COSY spectrum of 9 (DMSO- $d_{6}, 600 \mathrm{MHz}$ )

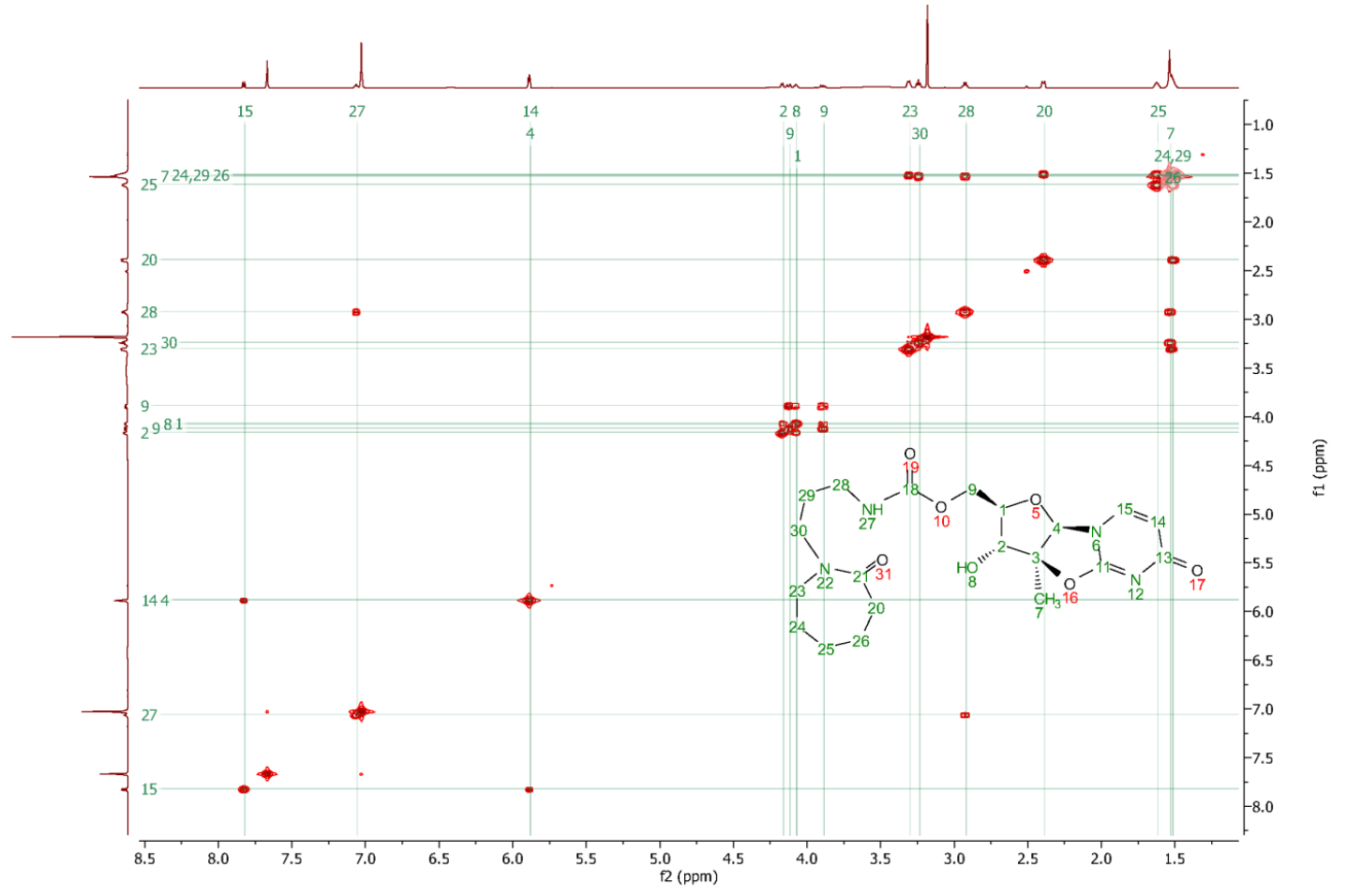


${ }^{1} \mathrm{H}-{ }^{13} \mathrm{C} \mathrm{HSQC}$ spectrum of 9 (DMSO- $d_{6}, 600 \mathrm{MHz}$ )

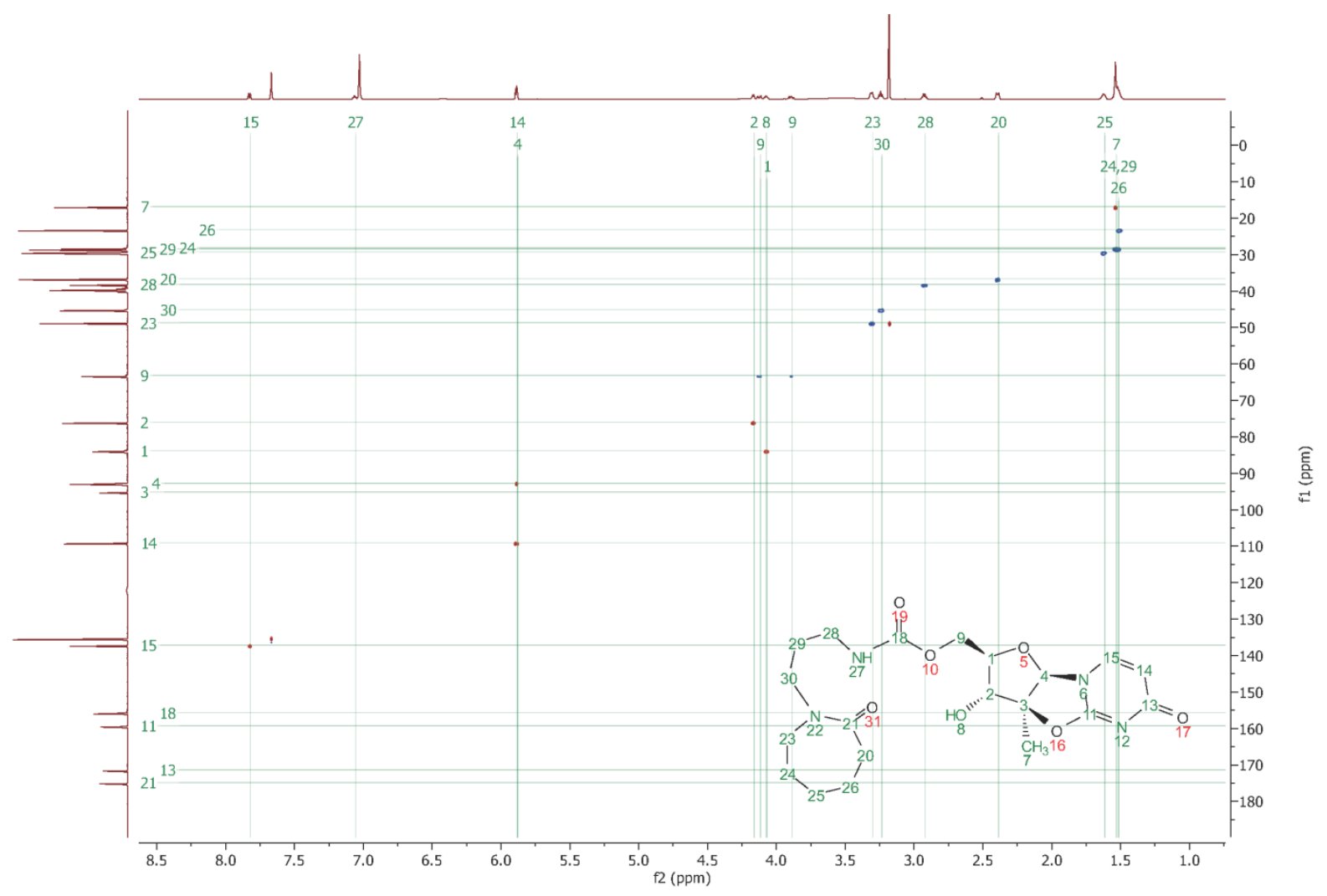

${ }^{1} \mathrm{H}-{ }^{13} \mathrm{C}$ HSQC-TOCSY spectrum of 9 (DMSO- $d_{6}, 600 \mathrm{MHz}$ )

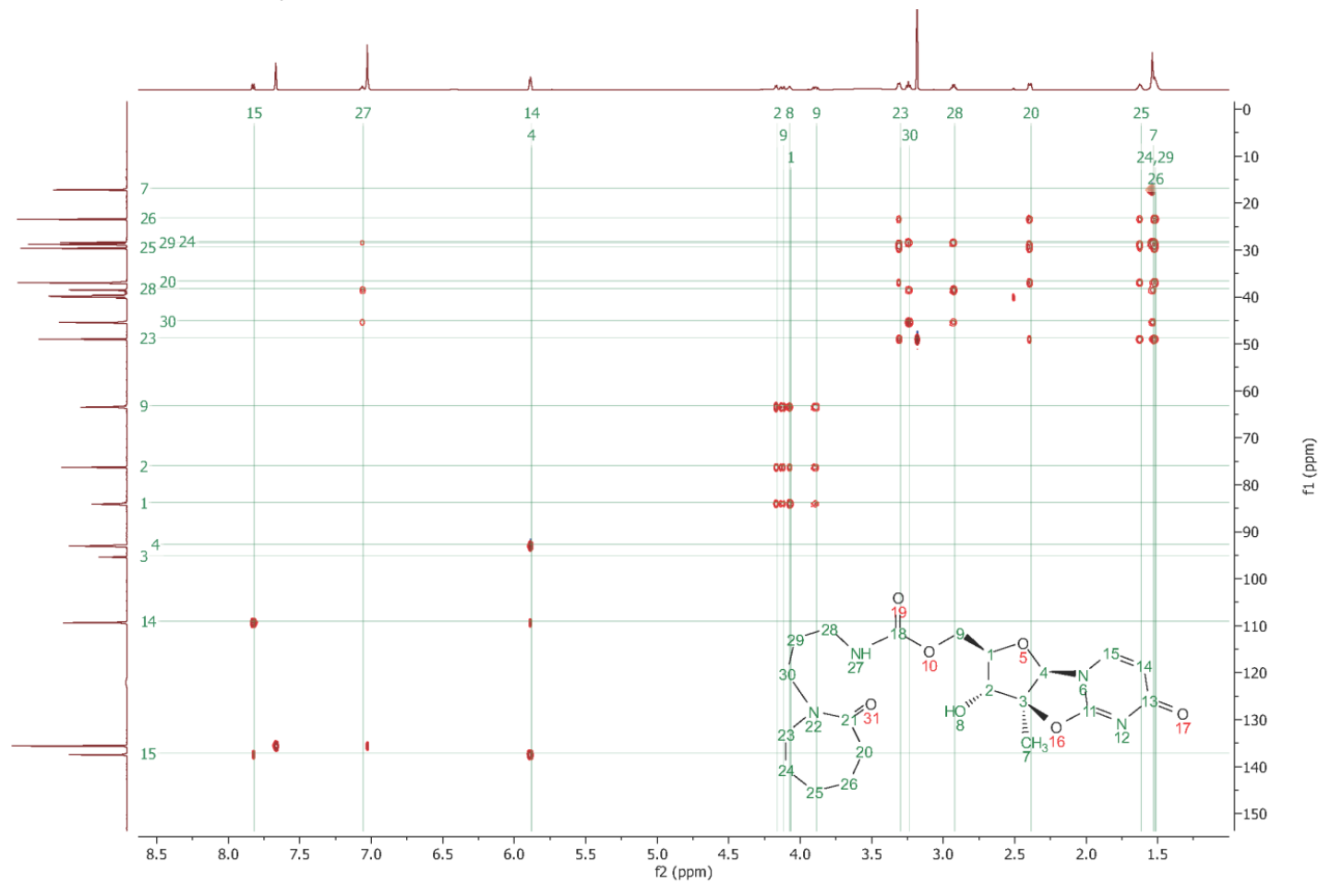


${ }^{1} \mathrm{H}-{ }^{13} \mathrm{C}$ HMBC spectrum of 9 (DMSO- $d_{6}, 600 \mathrm{MHz}$ )

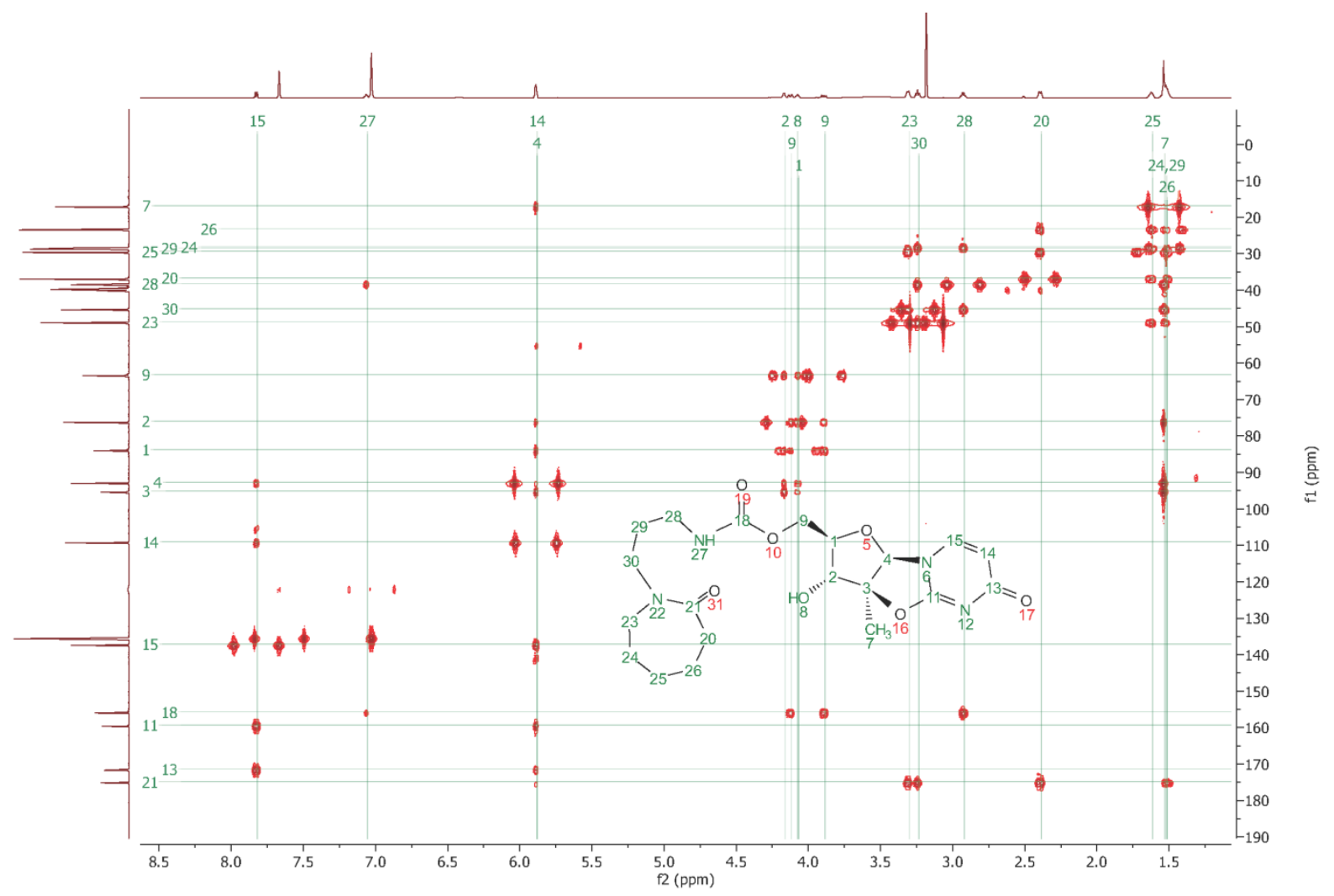

${ }^{1} \mathrm{H}-{ }^{15} \mathrm{~N} \mathrm{HMBC}$ spectrum of 9 (DMSO- $d_{6}, 600 \mathrm{MHz}$ )

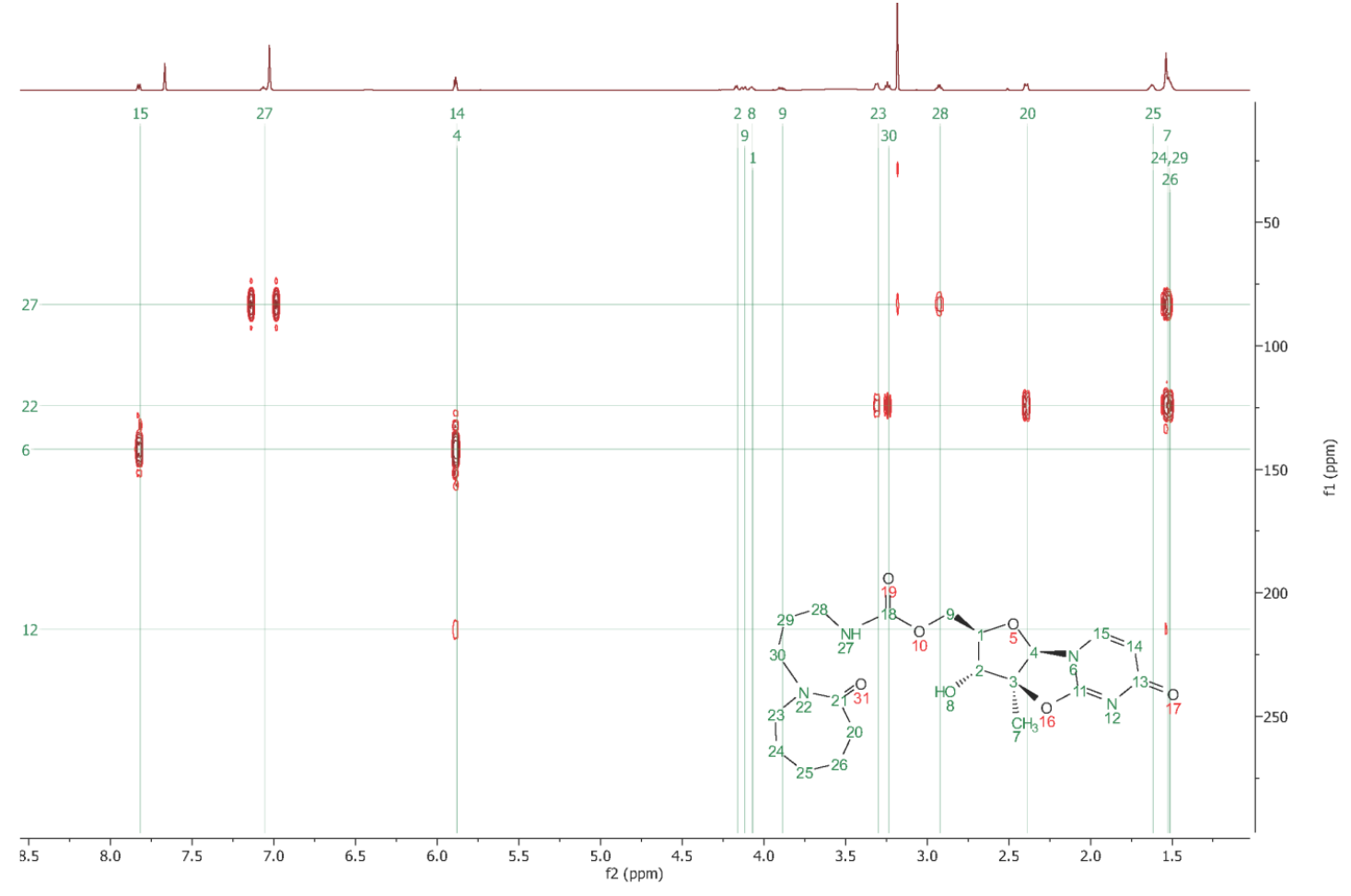


${ }^{1} \mathrm{H}-{ }^{1} \mathrm{H}$ ROESY spectrum of 9 (DMSO- $d_{6}, 600 \mathrm{MHz}$ )

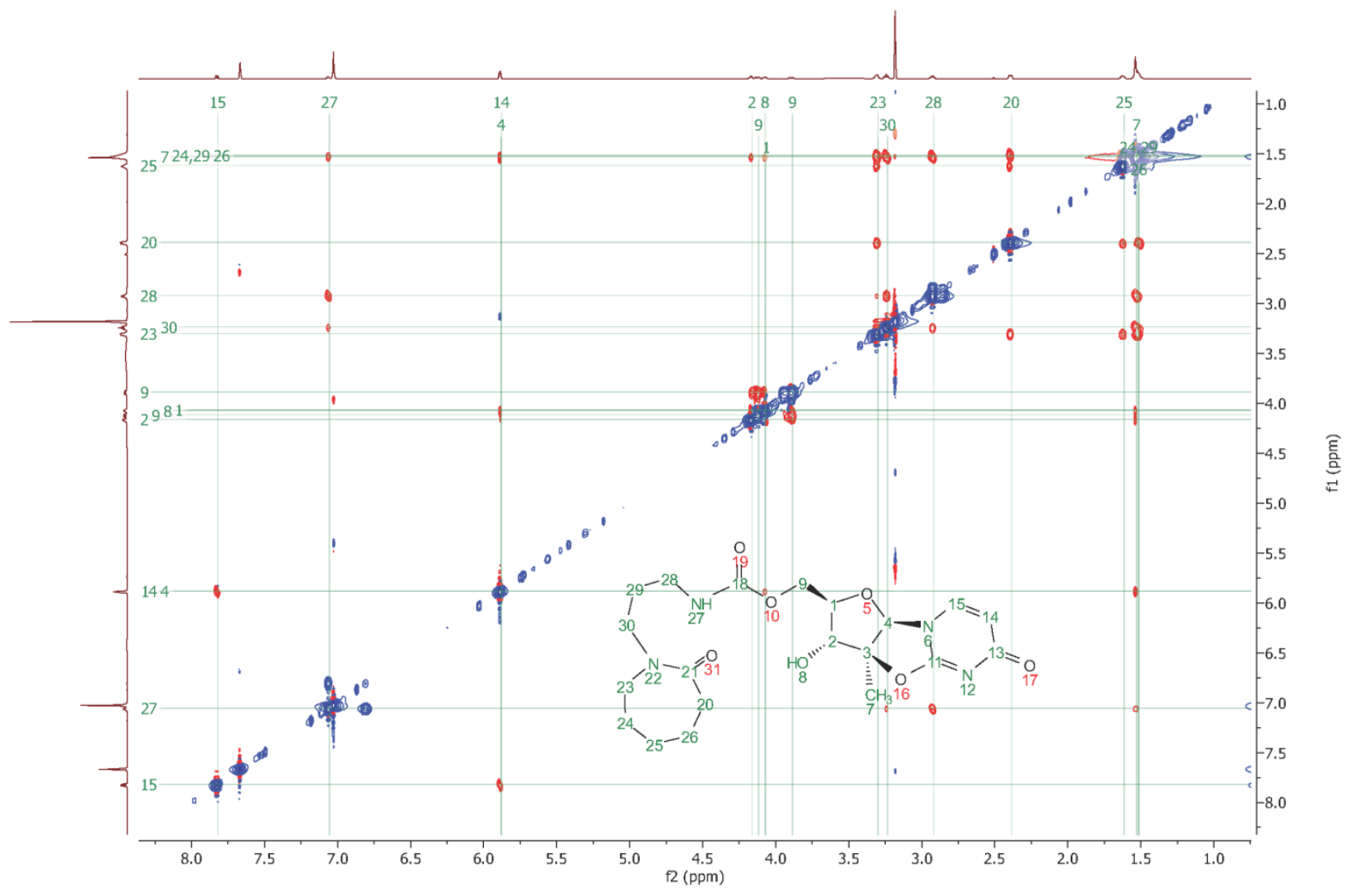


${ }^{1} \mathrm{H}$ spectrum of partial hydrolysis mixture of $\mathrm{DBU}\left(2: 1 \mathrm{D}_{2} \mathrm{O}: \mathrm{CD}_{3} \mathrm{CN}, 278 \mathrm{~K}, 600 \mathrm{MHz}\right)$

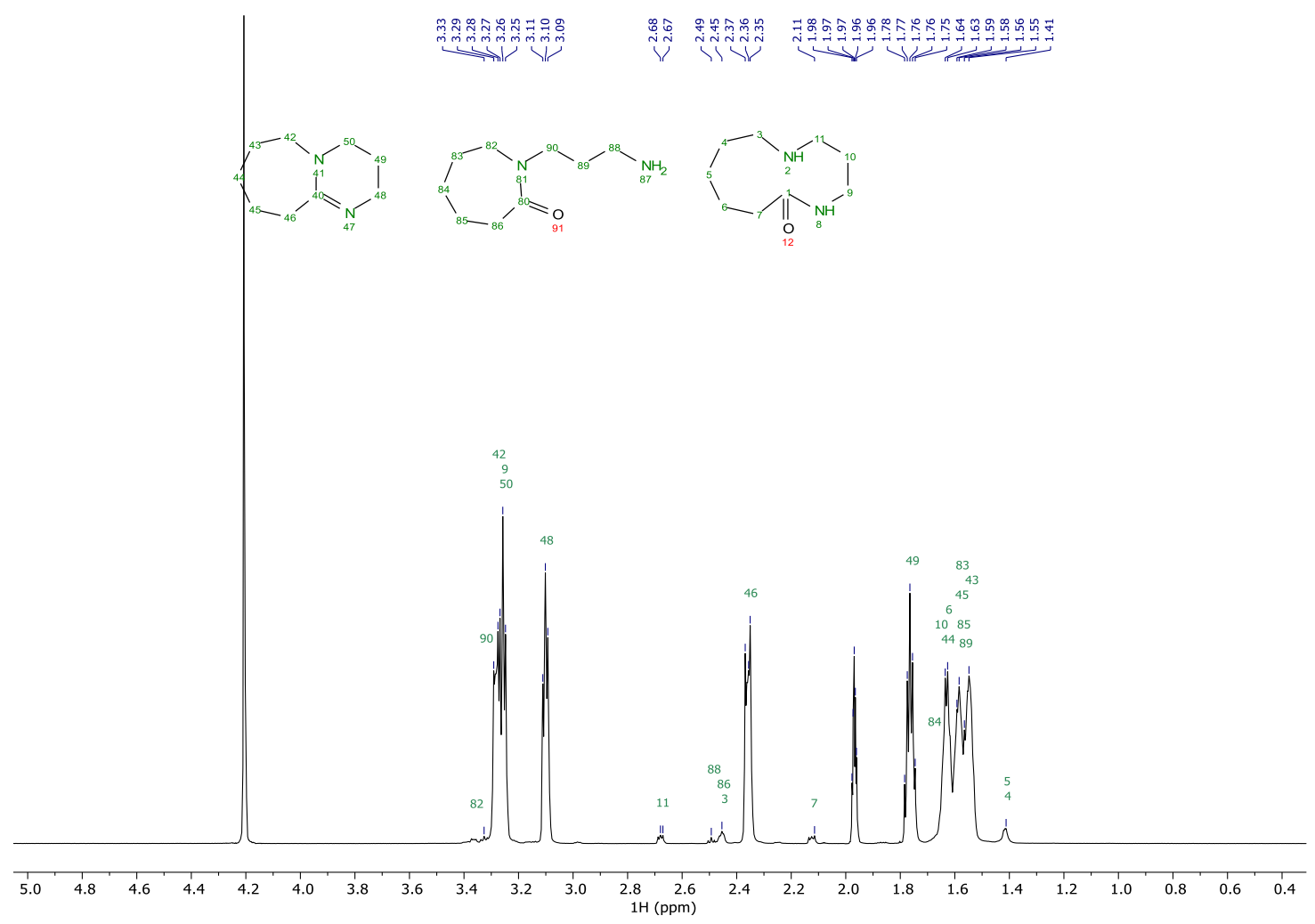

${ }^{1} \mathrm{H}-{ }^{1} \mathrm{H}$ COSY spectrum of partial hydrolysis mixture of DBU $\left(2: 1 \mathrm{D}_{2} \mathrm{O}: \mathrm{CD}_{3} \mathrm{CN}, 278 \mathrm{~K}, 600 \mathrm{MHz}\right)$

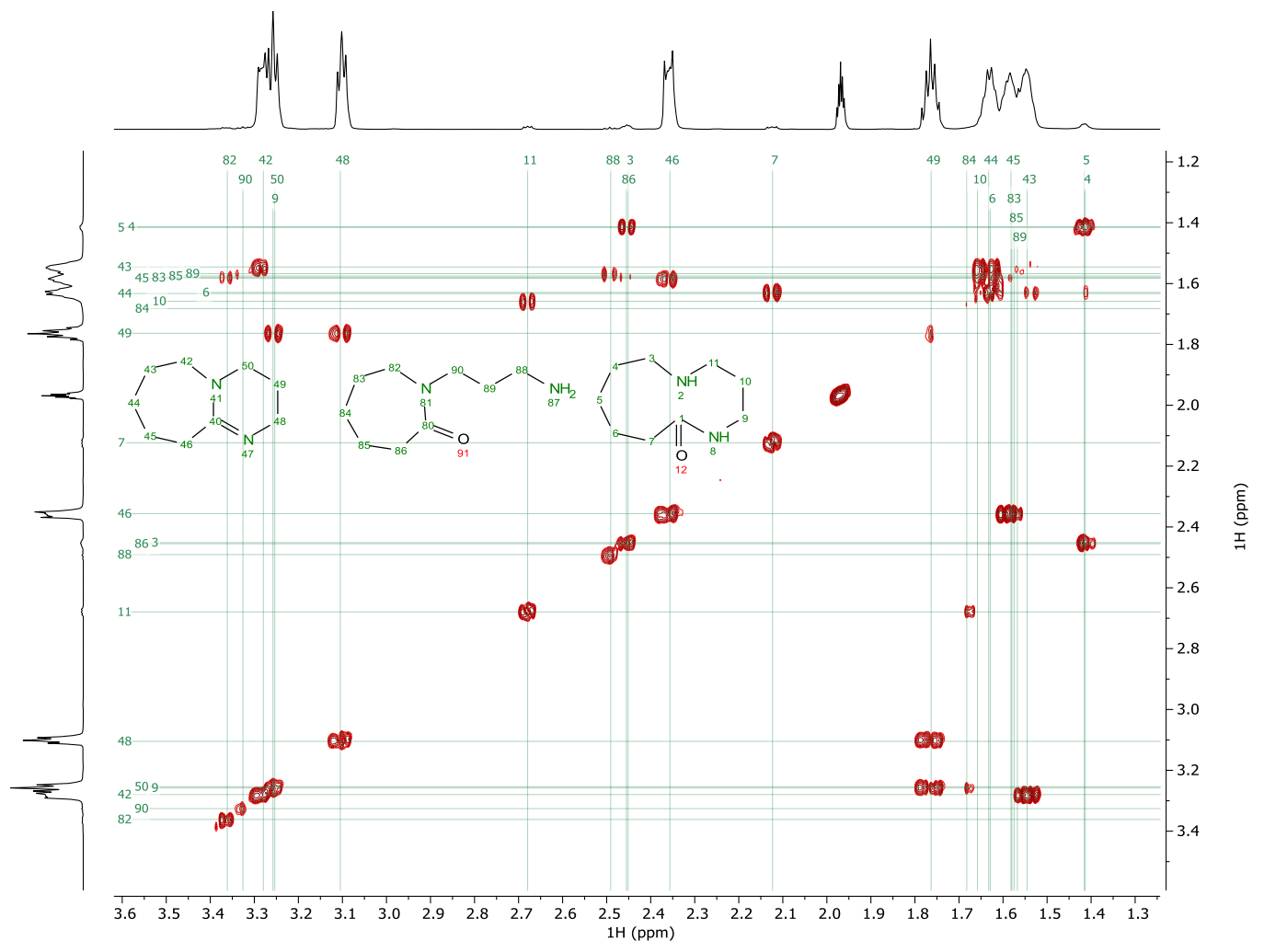


${ }^{1} \mathrm{H}-{ }^{1} \mathrm{H}$ TOCSY spectrum of partial hydrolysis mixture of $\mathrm{DBU}\left(2: 1 \mathrm{D}_{2} \mathrm{O}: \mathrm{CD}_{3} \mathrm{CN}, 278 \mathrm{~K}, 600 \mathrm{MHz}\right)$

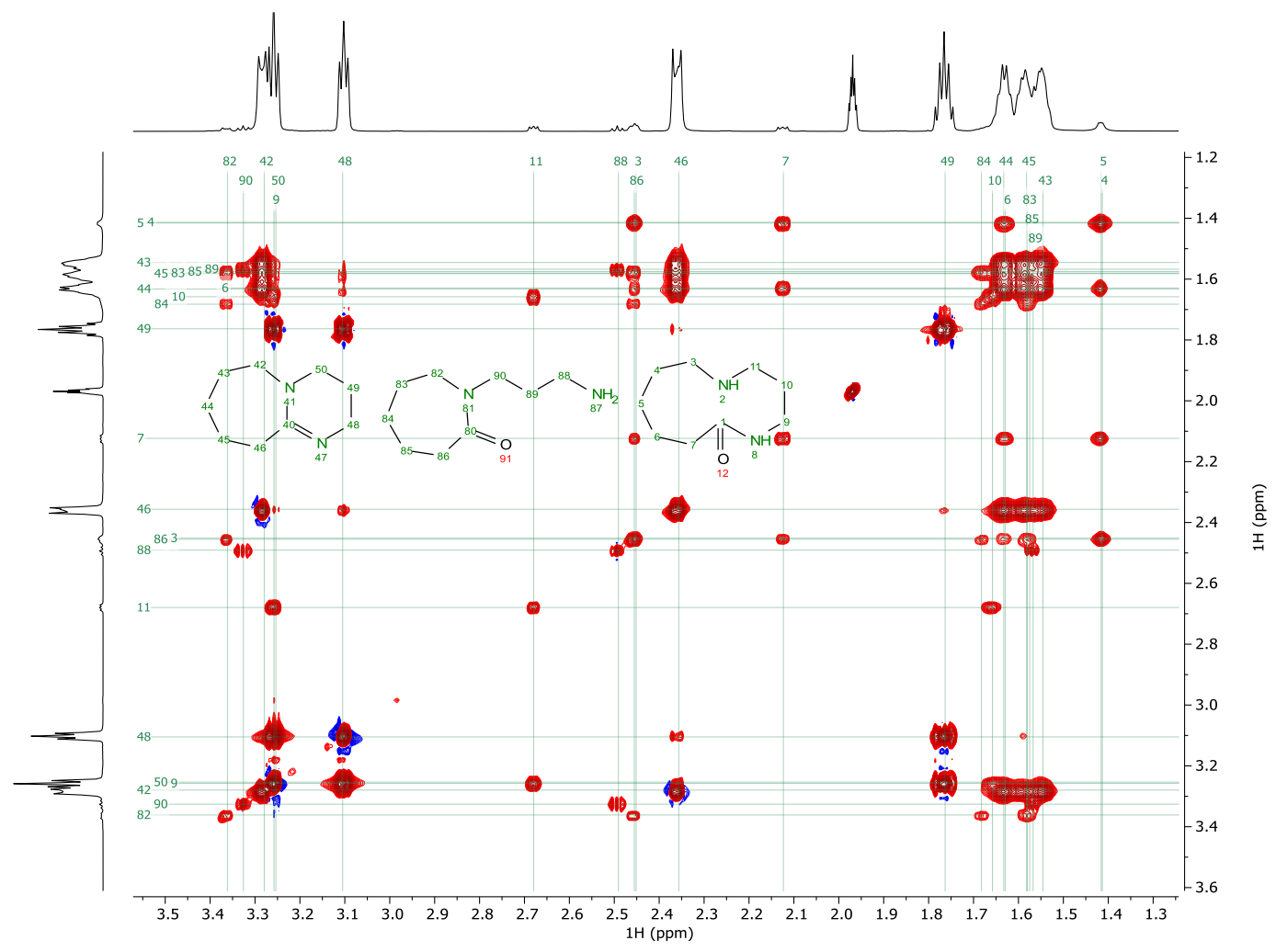

${ }^{1} \mathrm{H}-{ }^{13} \mathrm{C} \mathrm{HSQC}$ spectrum of partial hydrolysis mixture of $\mathrm{DBU}\left(2: 1 \mathrm{D}_{2} \mathrm{O}: \mathrm{CD}_{3} \mathrm{CN}, 278 \mathrm{~K}, 600 \mathrm{MHz}\right)$

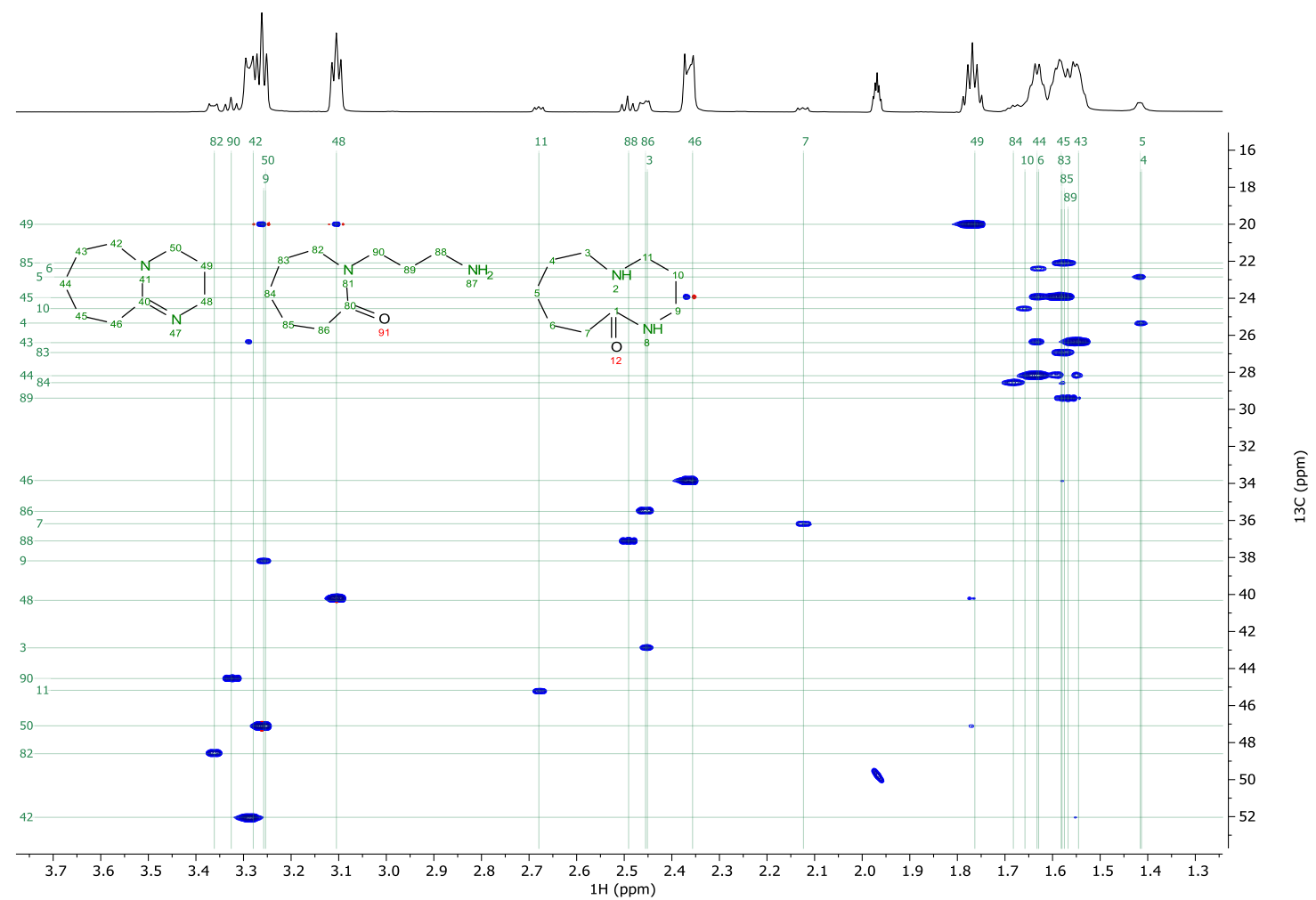


HSQC-TOCSY spectrum of partial hydrolysis mixture of DBU $\left(2: 1 \mathrm{D}_{2} \mathrm{O}: \mathrm{CD}_{3} \mathrm{CN}, 278 \mathrm{~K}, 600 \mathrm{MHz}\right)$

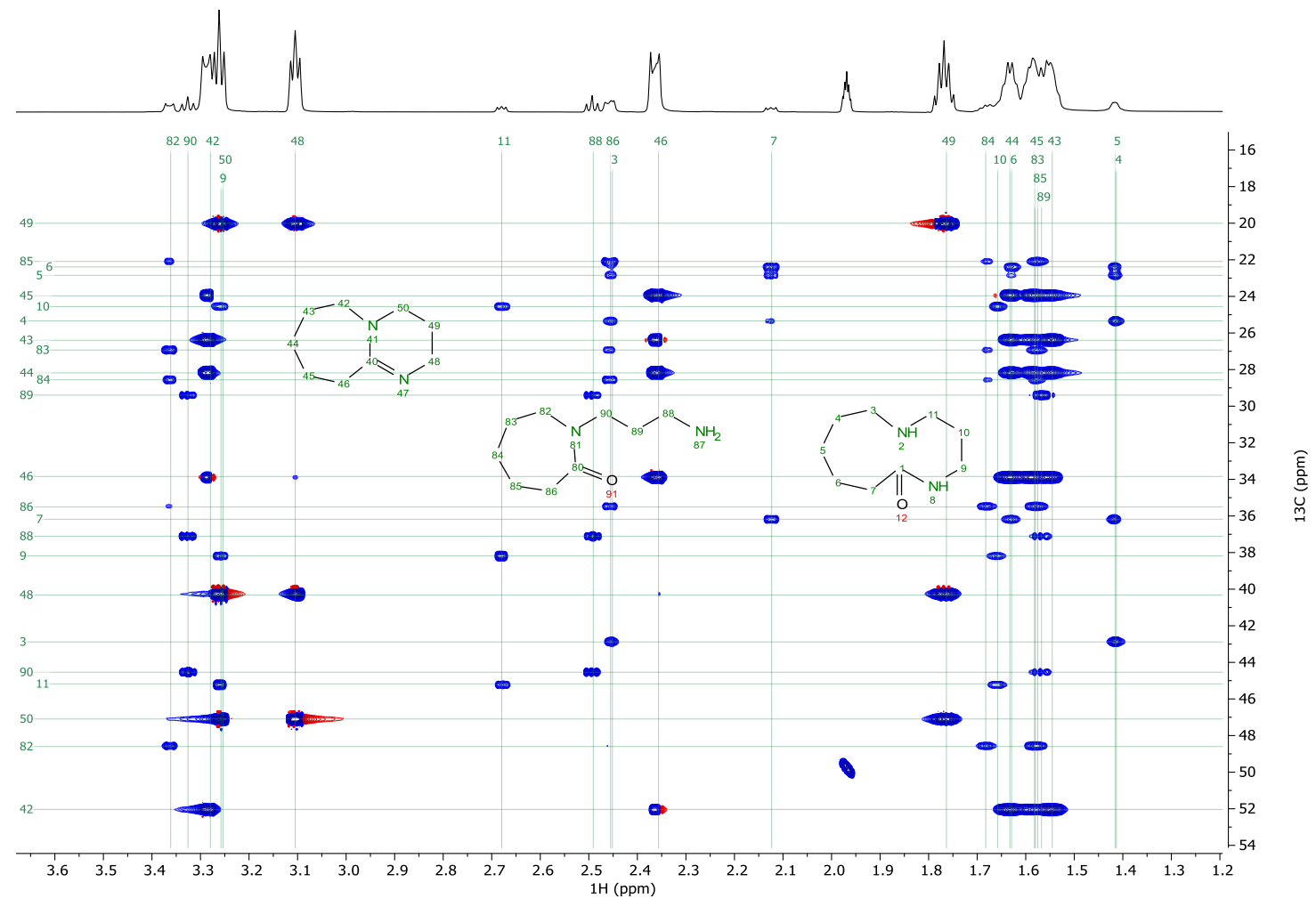

${ }^{1} \mathrm{H}-{ }^{13} \mathrm{C} \mathrm{HMBC}$ spectrum of partial hydrolysis mixture of $\mathrm{DBU}\left(2: 1 \mathrm{D}_{2} \mathrm{O}: \mathrm{CD}_{3} \mathrm{CN}, 278 \mathrm{~K}, 600 \mathrm{MHz}\right)$

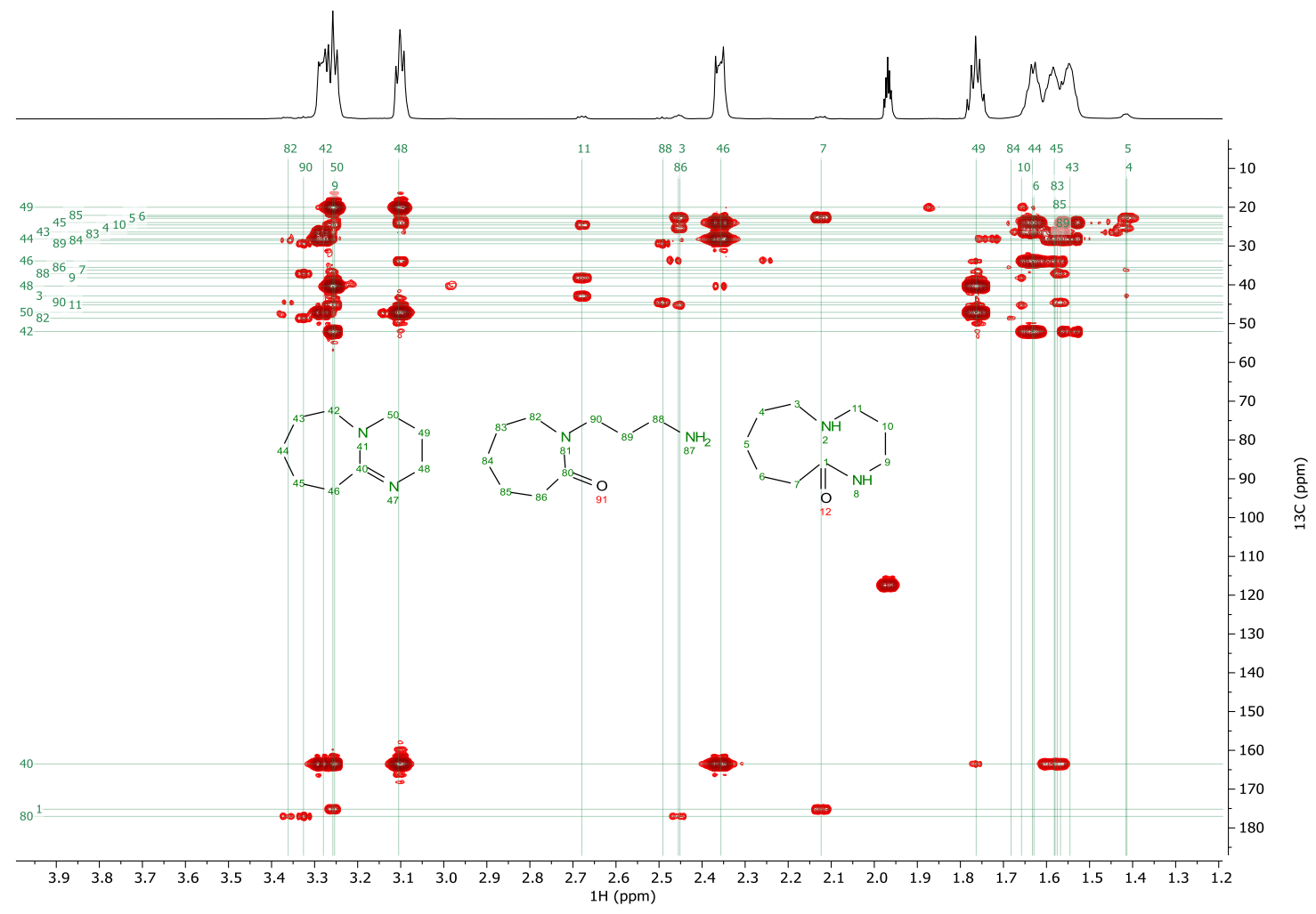


${ }^{1} \mathrm{H}-{ }^{15} \mathrm{~N} \mathrm{HMBC}$ spectrum of partial hydrolysis mixture of $\mathrm{DBU}\left(2: 1 \mathrm{D}_{2} \mathrm{O}: \mathrm{CD}_{3} \mathrm{CN}, 278 \mathrm{~K}, 600 \mathrm{MHz}\right)$

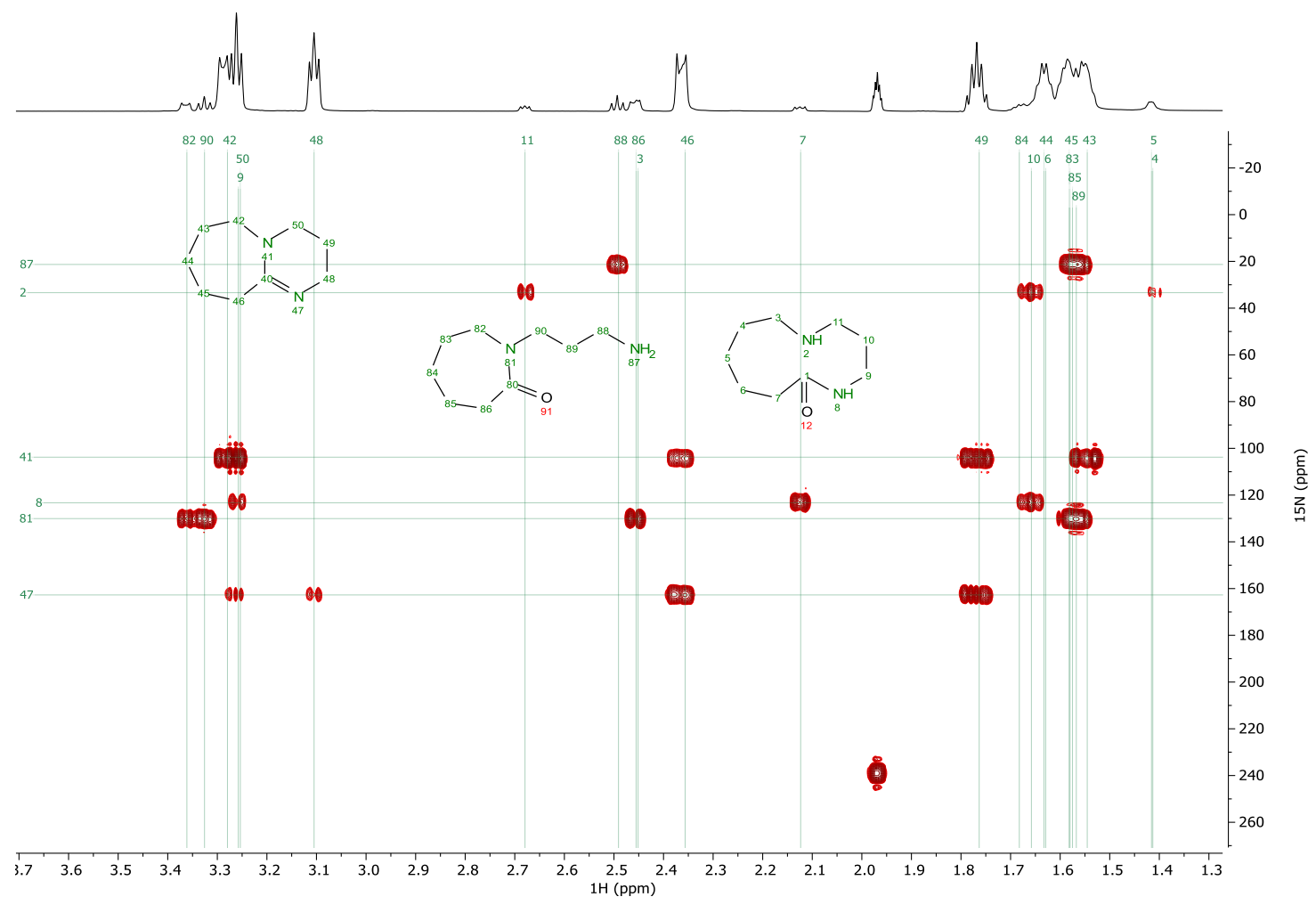

${ }^{1} \mathrm{H}$ spectrum of partial hydrolysis mixture of $\operatorname{DBN}\left(\mathrm{D}_{2} \mathrm{O}, 500 \mathrm{MHz}\right)$

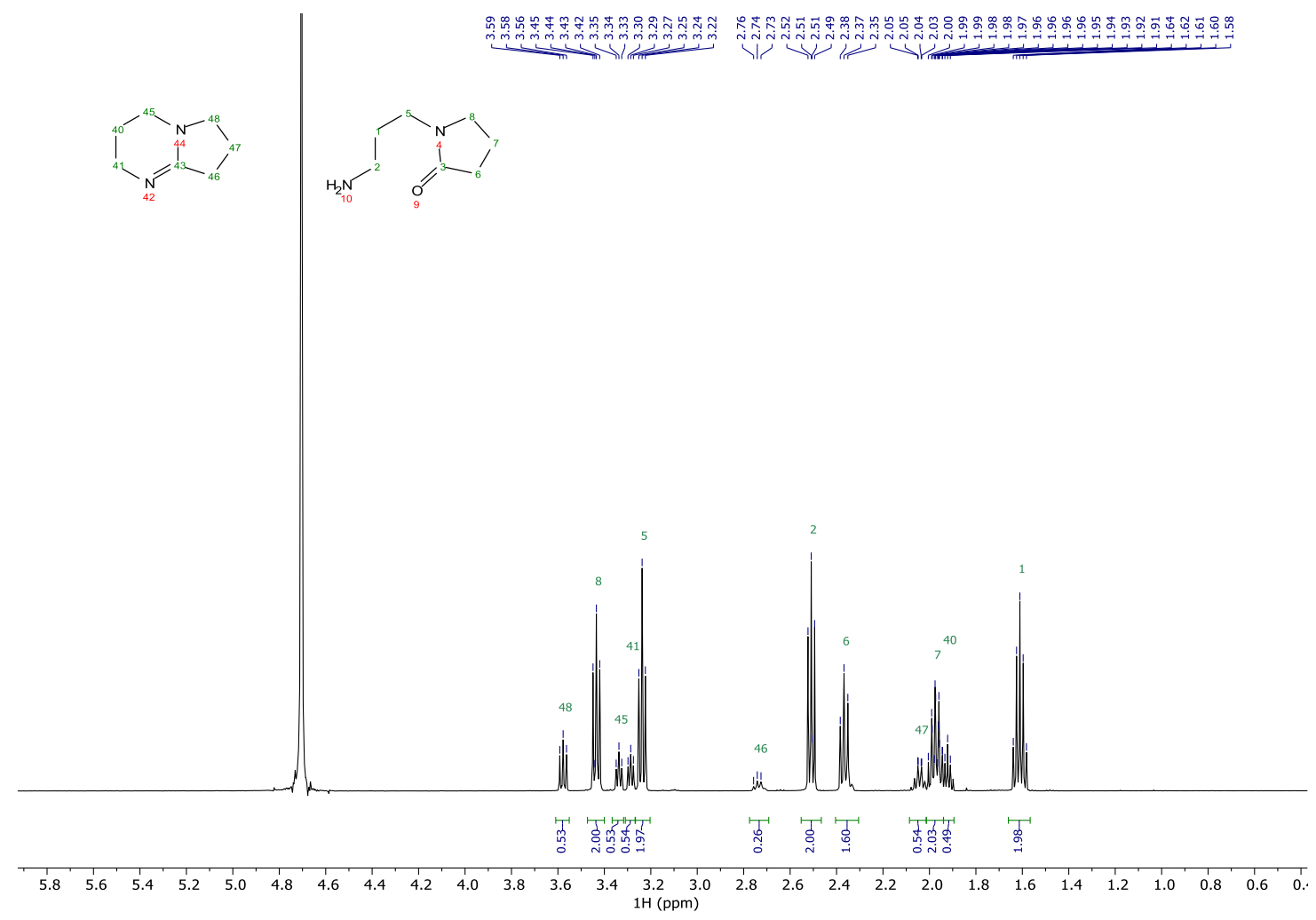


${ }^{13} \mathrm{C}\left\{{ }^{1} \mathrm{H}\right\}$ spectrum of partial hydrolysis mixture of DBNG $\left(\mathrm{D}_{2} \mathrm{O}, 126 \mathrm{MHz}\right)$
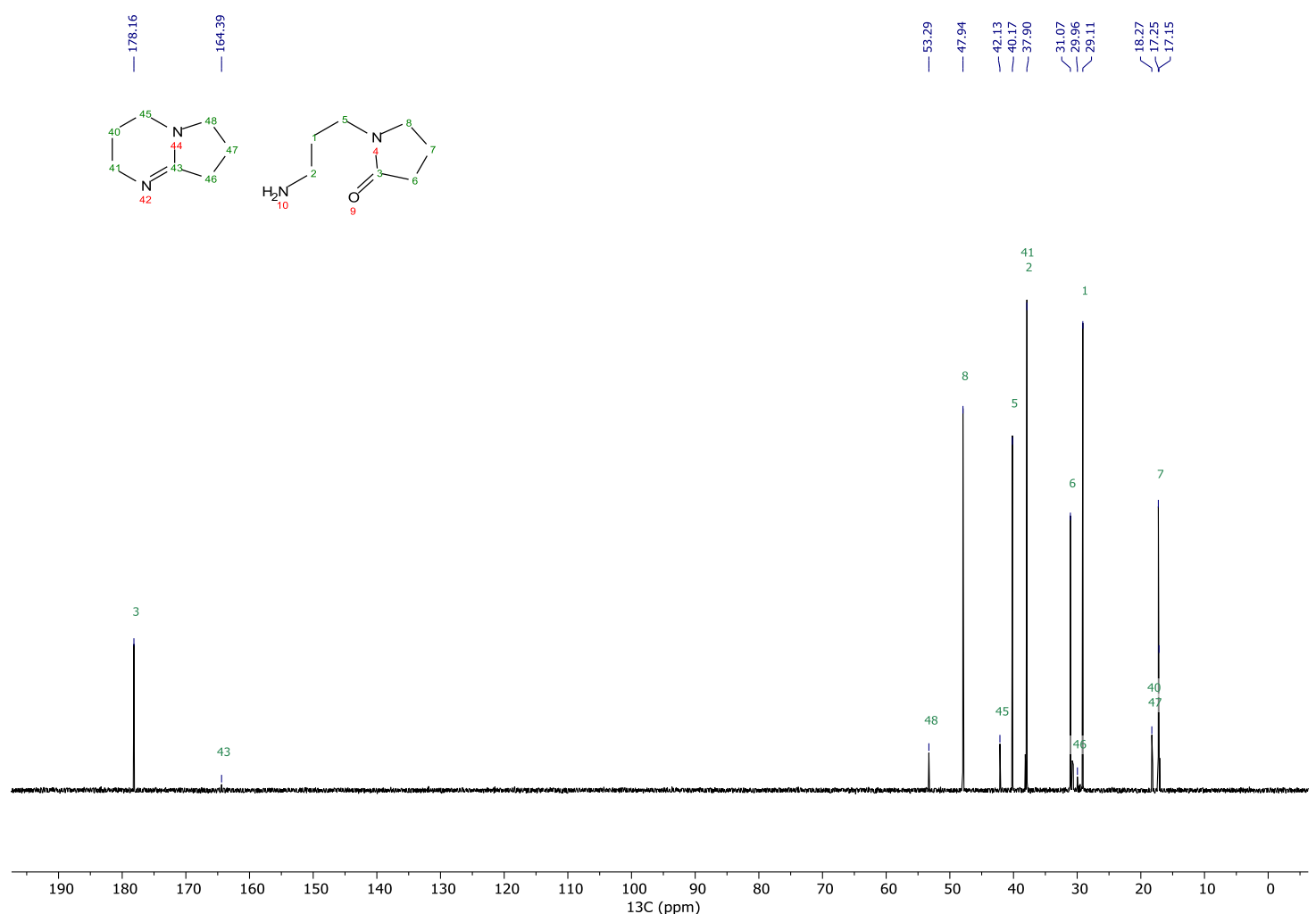

${ }^{1} \mathrm{H}-{ }^{1} \mathrm{H}$ COSY spectrum of partial hydrolysis mixture of $\mathrm{DBN}\left(\mathrm{D}_{2} \mathrm{O}, 500 \mathrm{MHz}\right)$

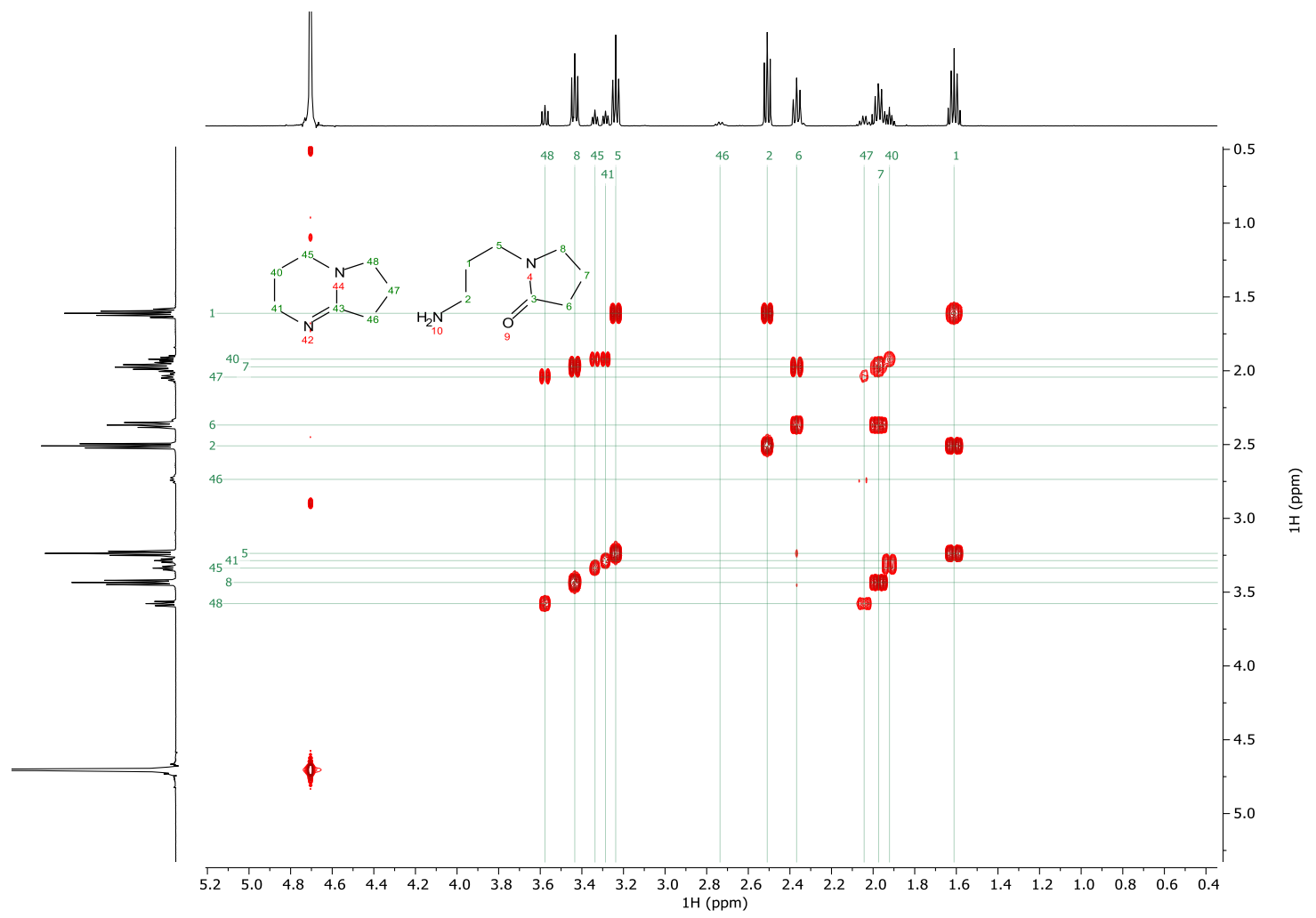


${ }^{1} \mathrm{H}-{ }^{13} \mathrm{C} \mathrm{HSQC}$ spectrum of partial hydrolysis mixture of $\mathrm{DBN}\left(\mathrm{D}_{2} \mathrm{O}, 500 \mathrm{MHz}\right)$

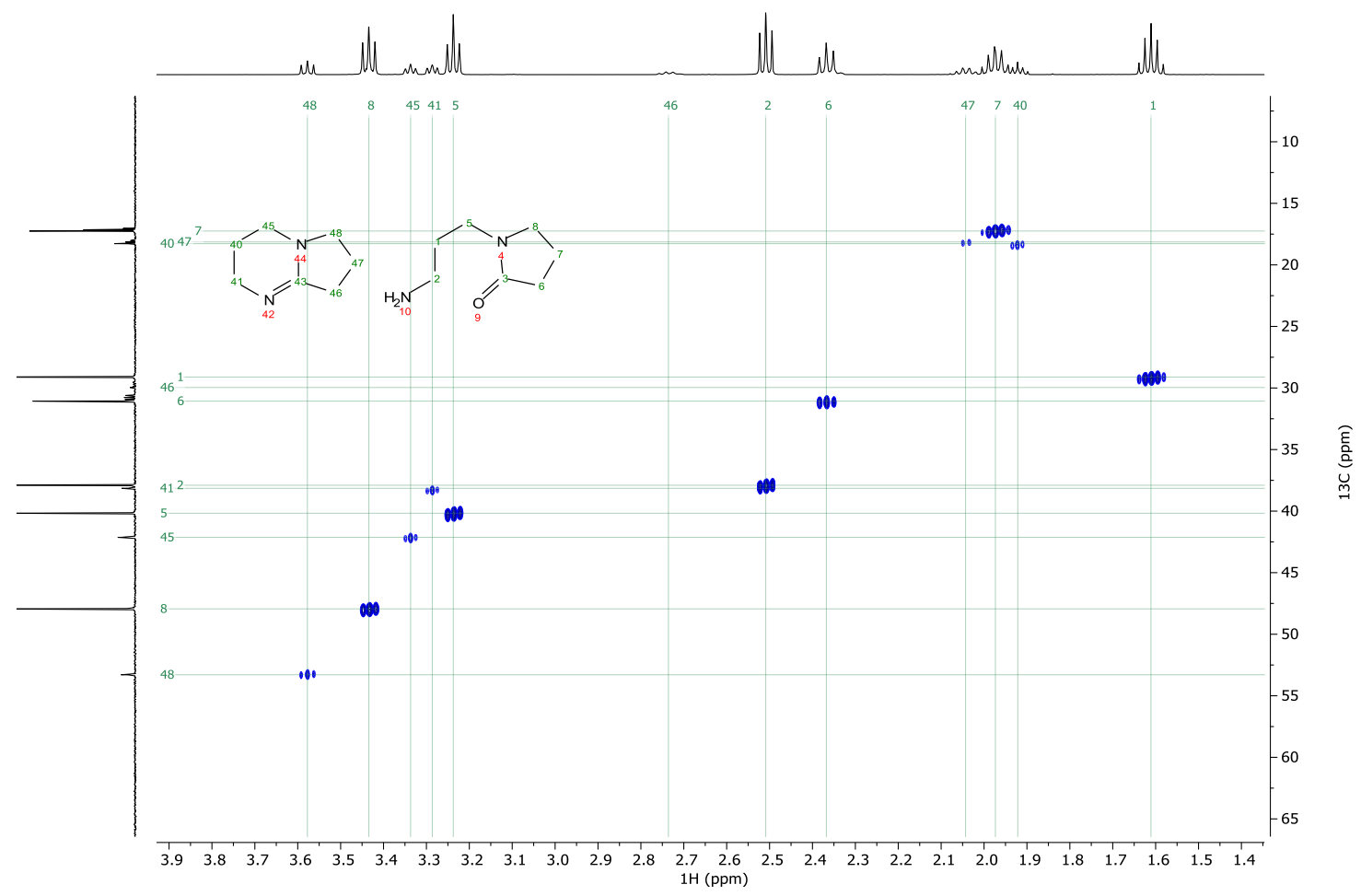

${ }^{1} \mathrm{H}-{ }^{13} \mathrm{C} \mathrm{HMBC}$ spectrum of partial hydrolysis mixture of $\mathrm{DBN}\left(\mathrm{D}_{2} \mathrm{O}, 500 \mathrm{MHz}\right)$

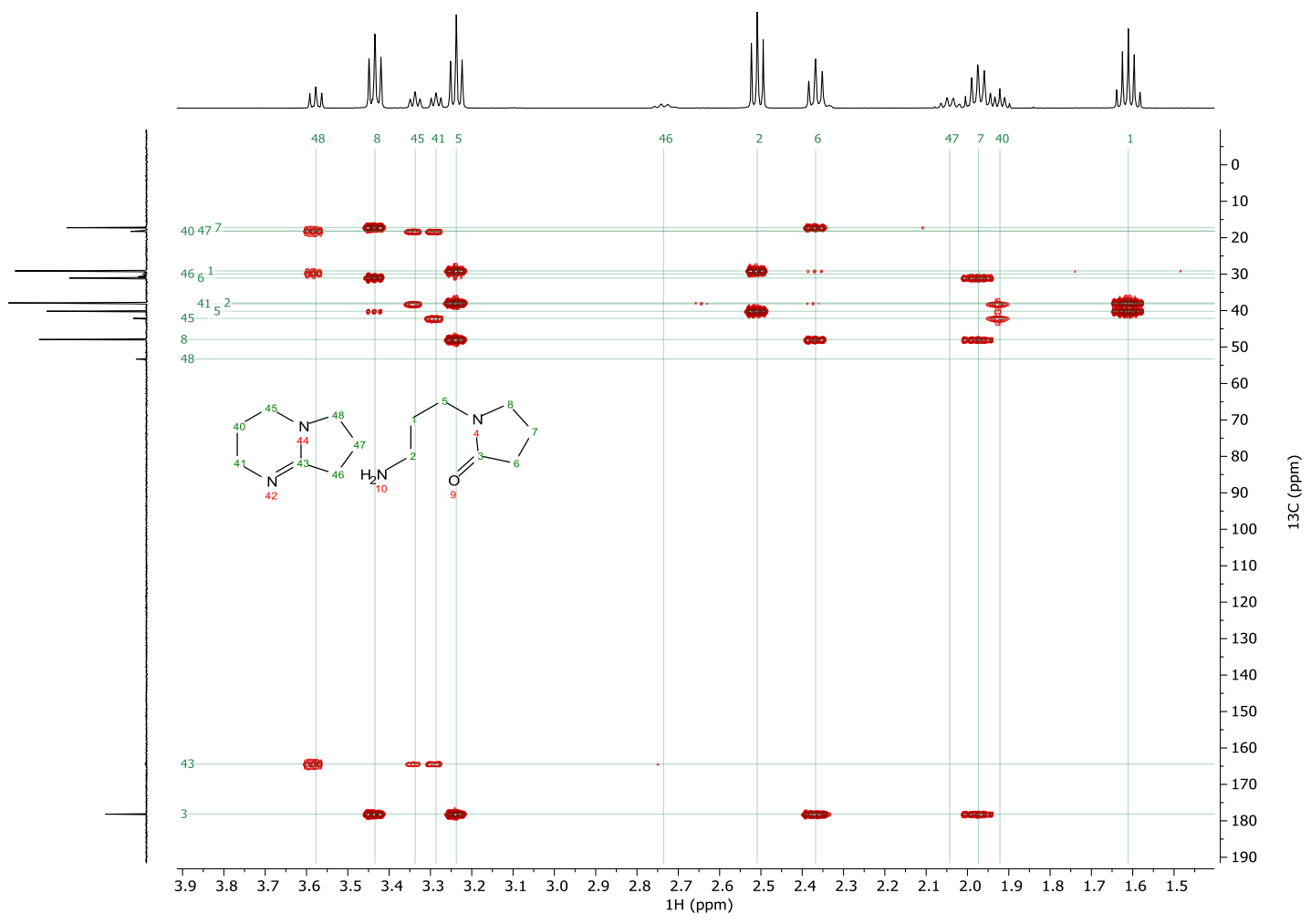


${ }^{1} \mathrm{H}-{ }^{1} \mathrm{H}$ TOCSY spectrum of partial hydrolysis mixture of $\mathrm{DBN}\left(\mathrm{D}_{2} \mathrm{O}, 500 \mathrm{MHz}\right)$

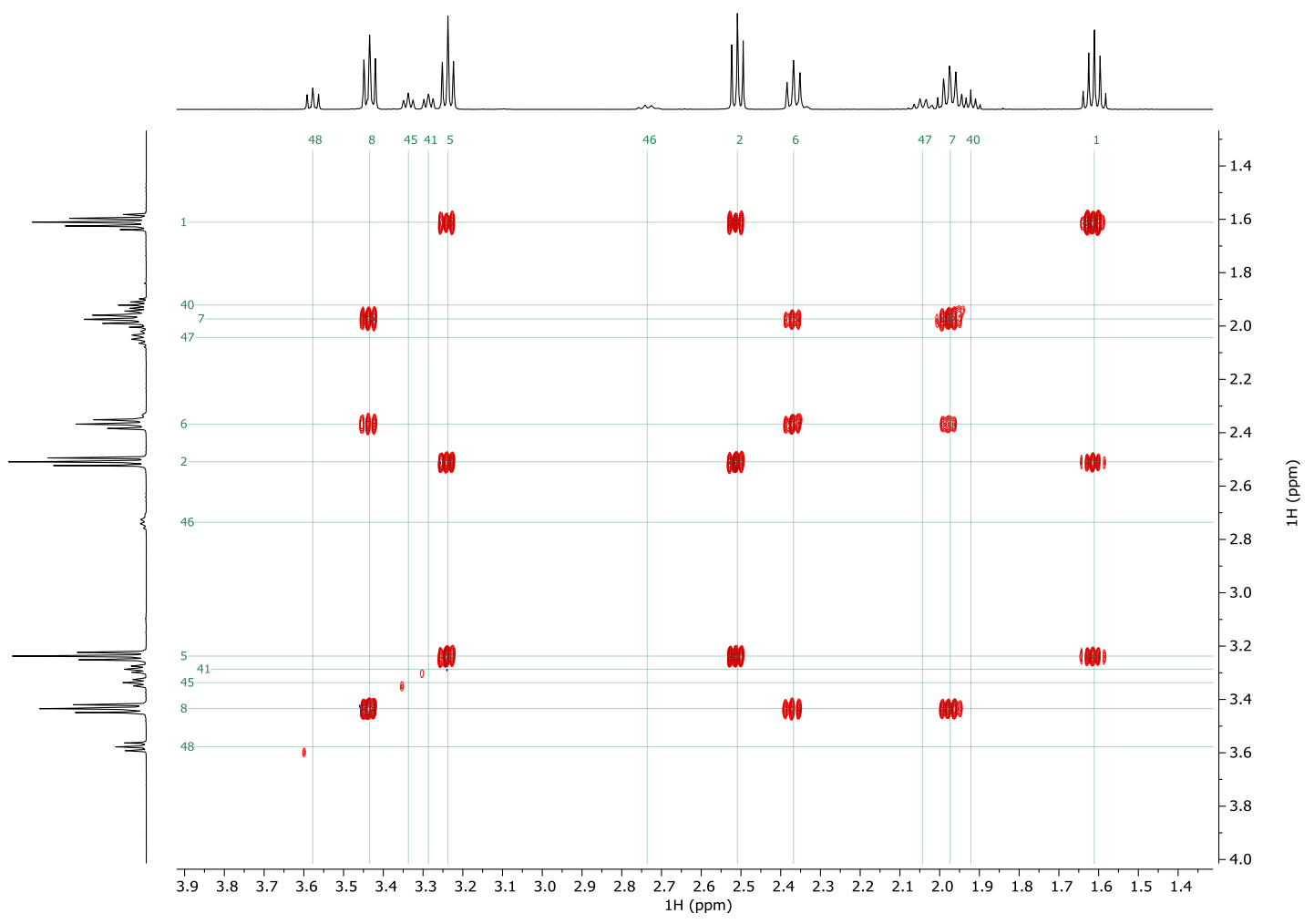

HSQC-TOCSY spectrum of partial hydrolysis mixture of DBN $\left(D_{2} \mathrm{O}, 500 \mathrm{MHz}\right)$

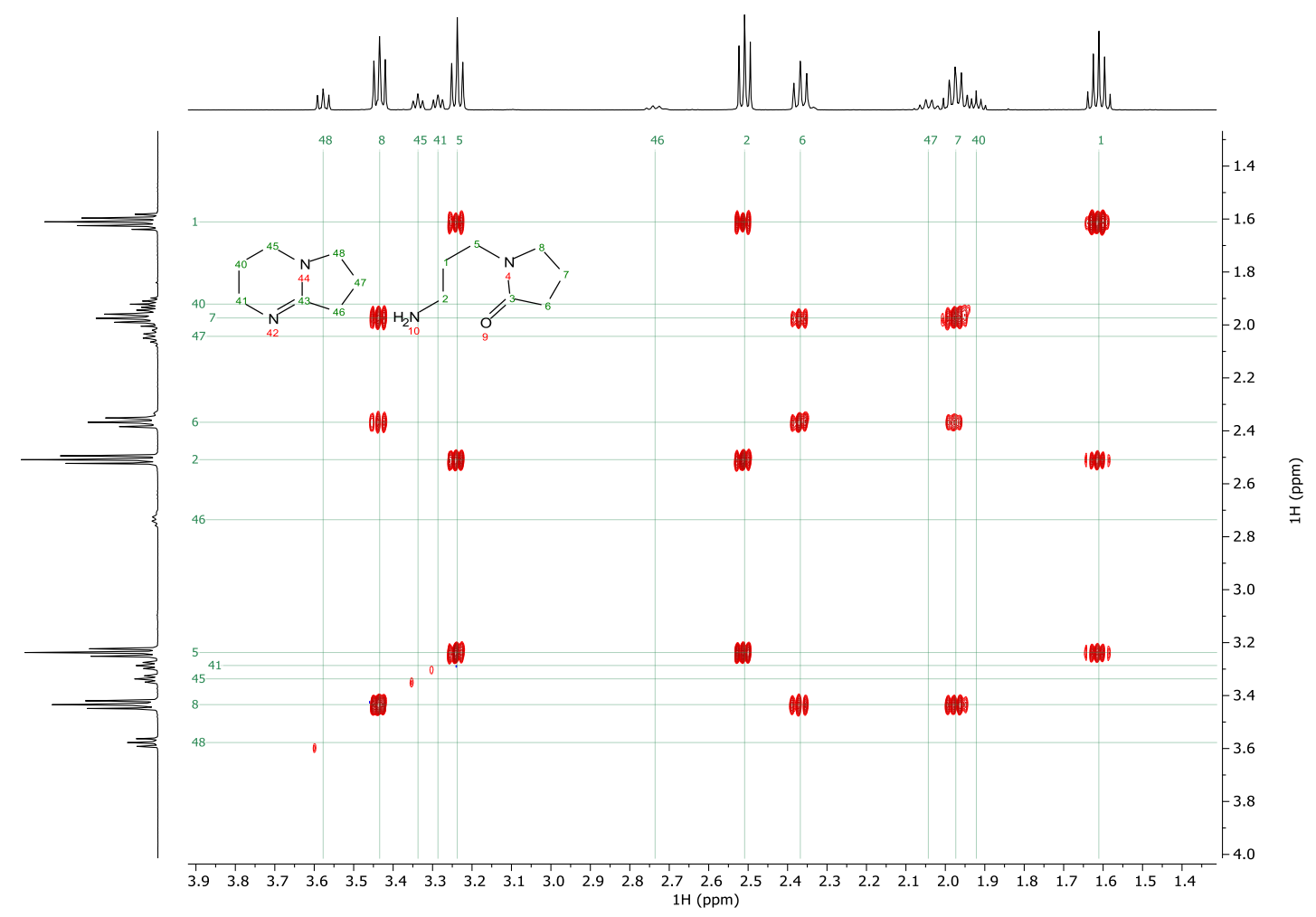


${ }^{1} \mathrm{H}$ spectrum of partial hydrolysis mixture of $\operatorname{MTBD}\left(\mathrm{D}_{2} \mathrm{O}, 600 \mathrm{MHz}\right)$

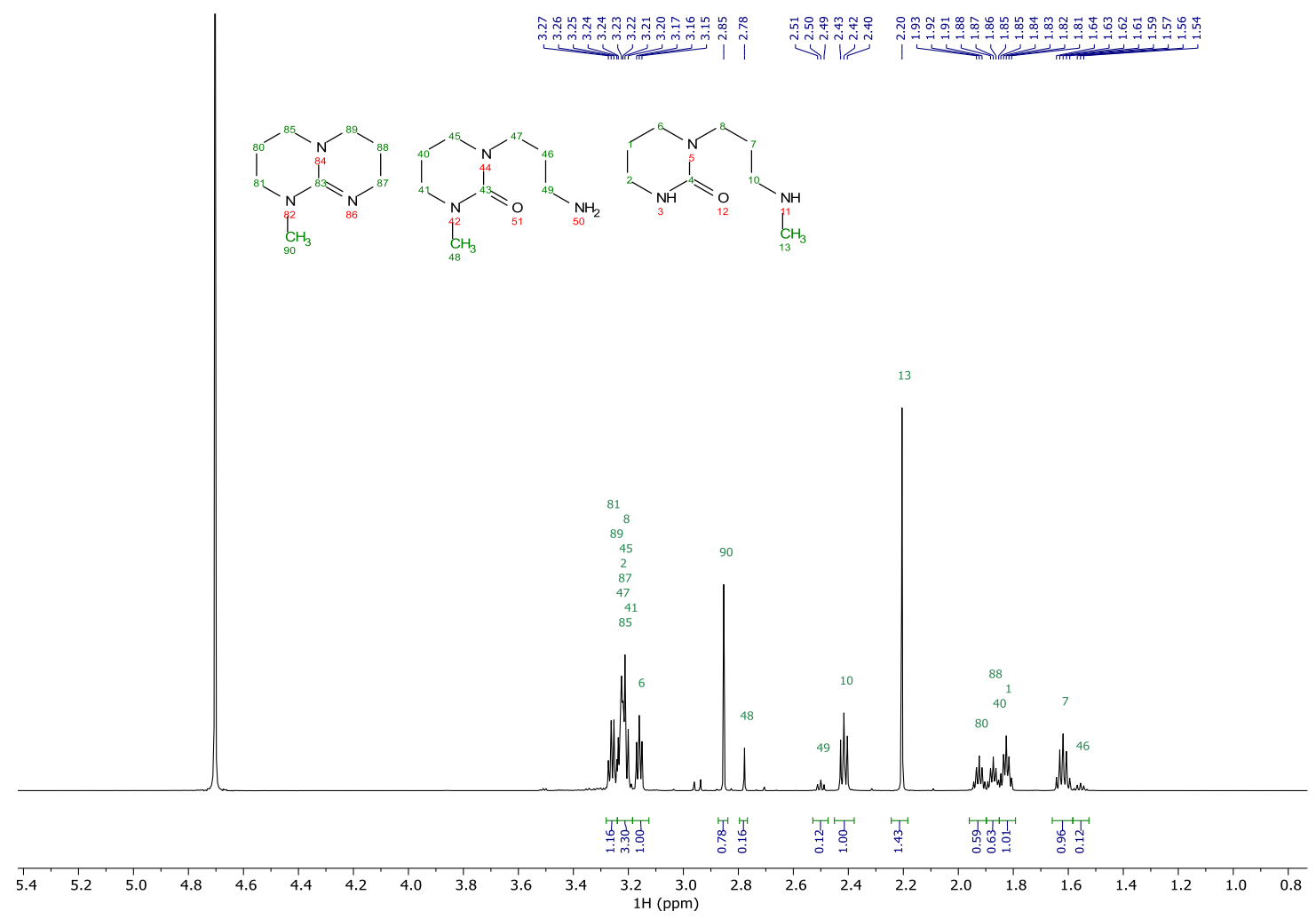

${ }^{1} \mathrm{H}-{ }^{1} \mathrm{H}$ COSY spectrum of partial hydrolysis mixture of $\operatorname{MTBD}\left(\mathrm{D}_{2} \mathrm{O}, 600 \mathrm{MHz}\right)$

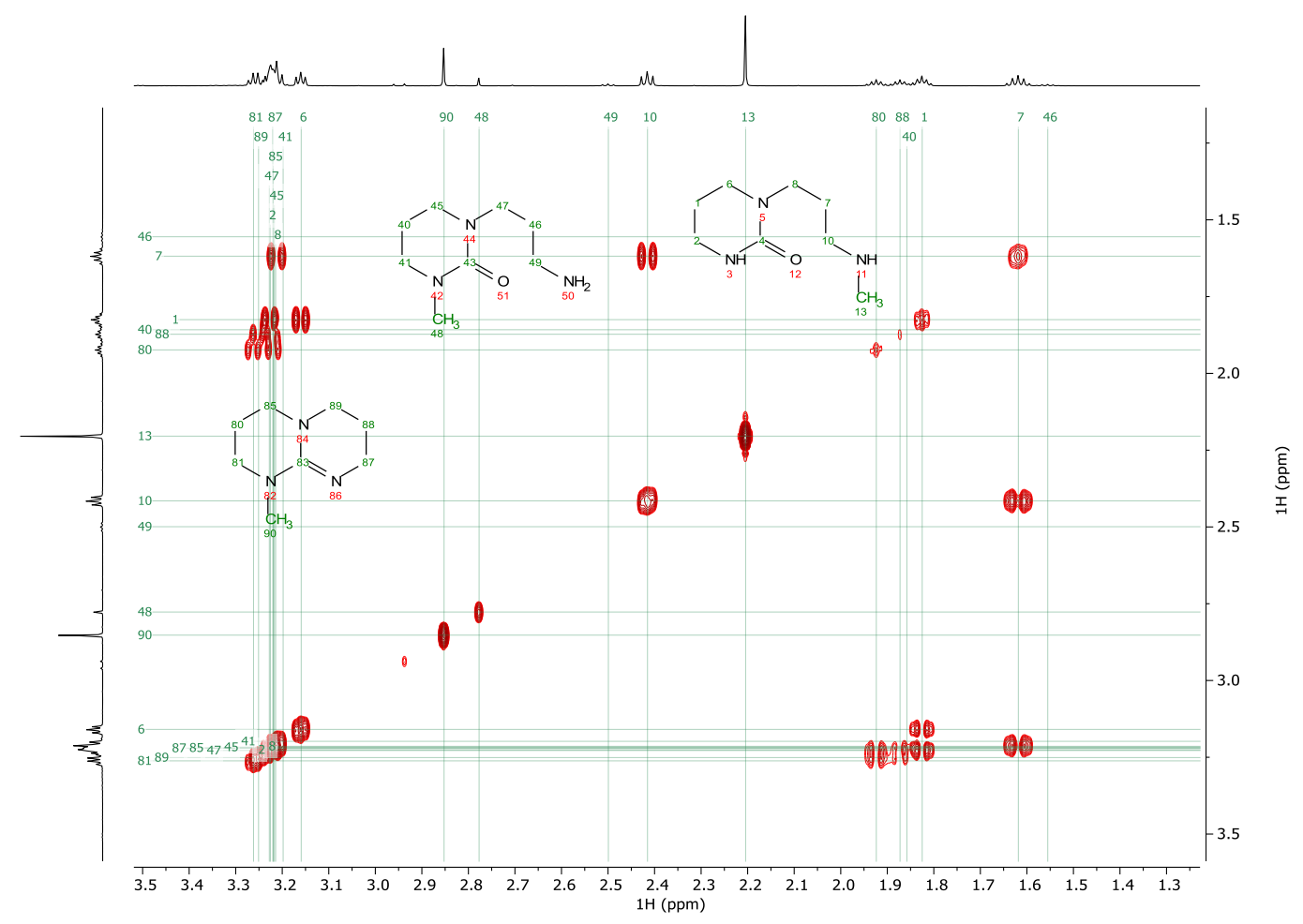


${ }^{1} \mathrm{H}-{ }^{13} \mathrm{C} \mathrm{HSQC}$ spectrum of partial hydrolysis mixture of $\operatorname{MTBD}\left(\mathrm{D}_{2} \mathrm{O}, 600 \mathrm{MHz}\right)$

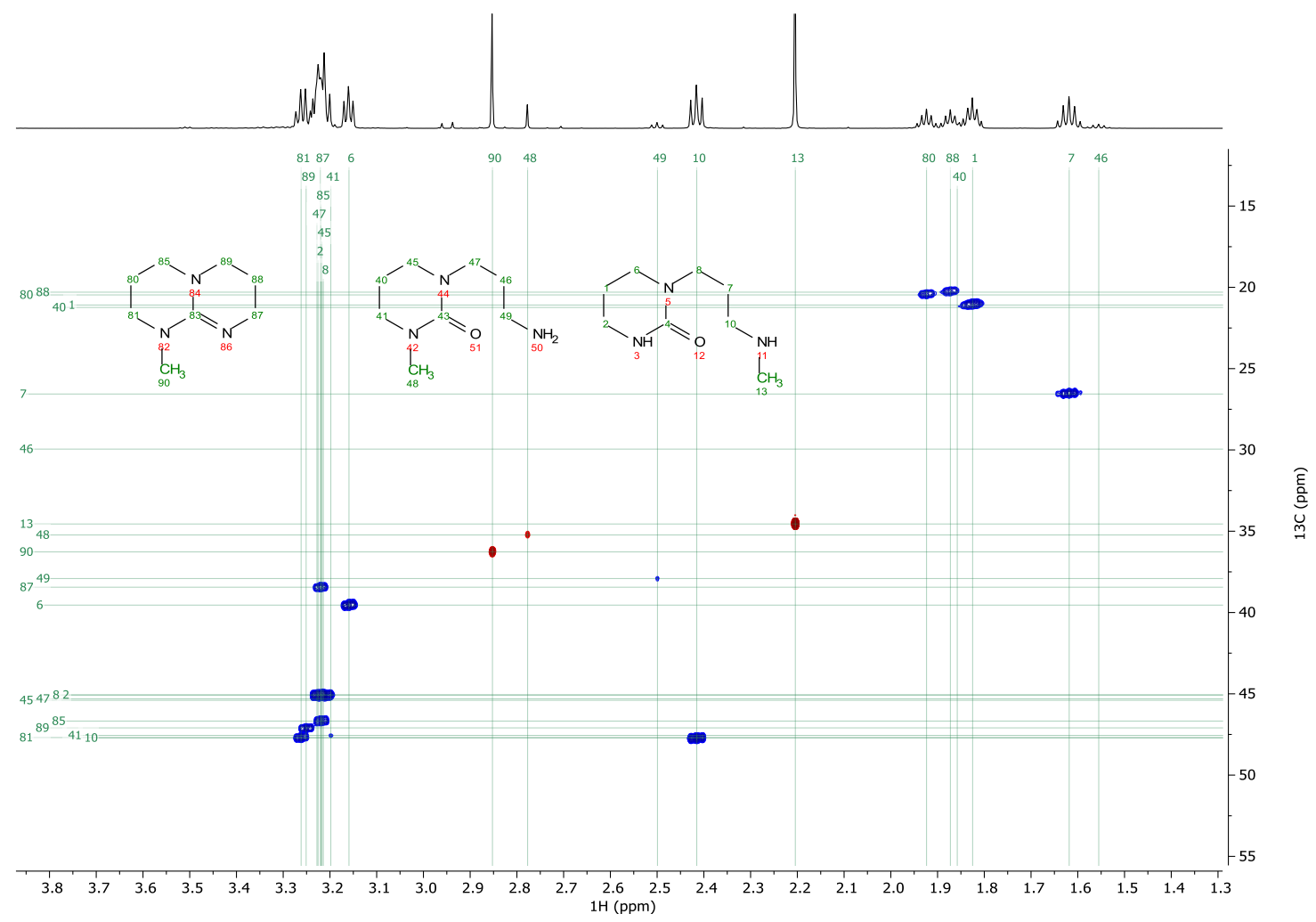

${ }^{1} \mathrm{H}-{ }^{13} \mathrm{C}$ HMBC spectrum of partial hydrolysis mixture of $\operatorname{MTBD}\left(\mathrm{D}_{2} \mathrm{O}, 600 \mathrm{MHz}\right)$

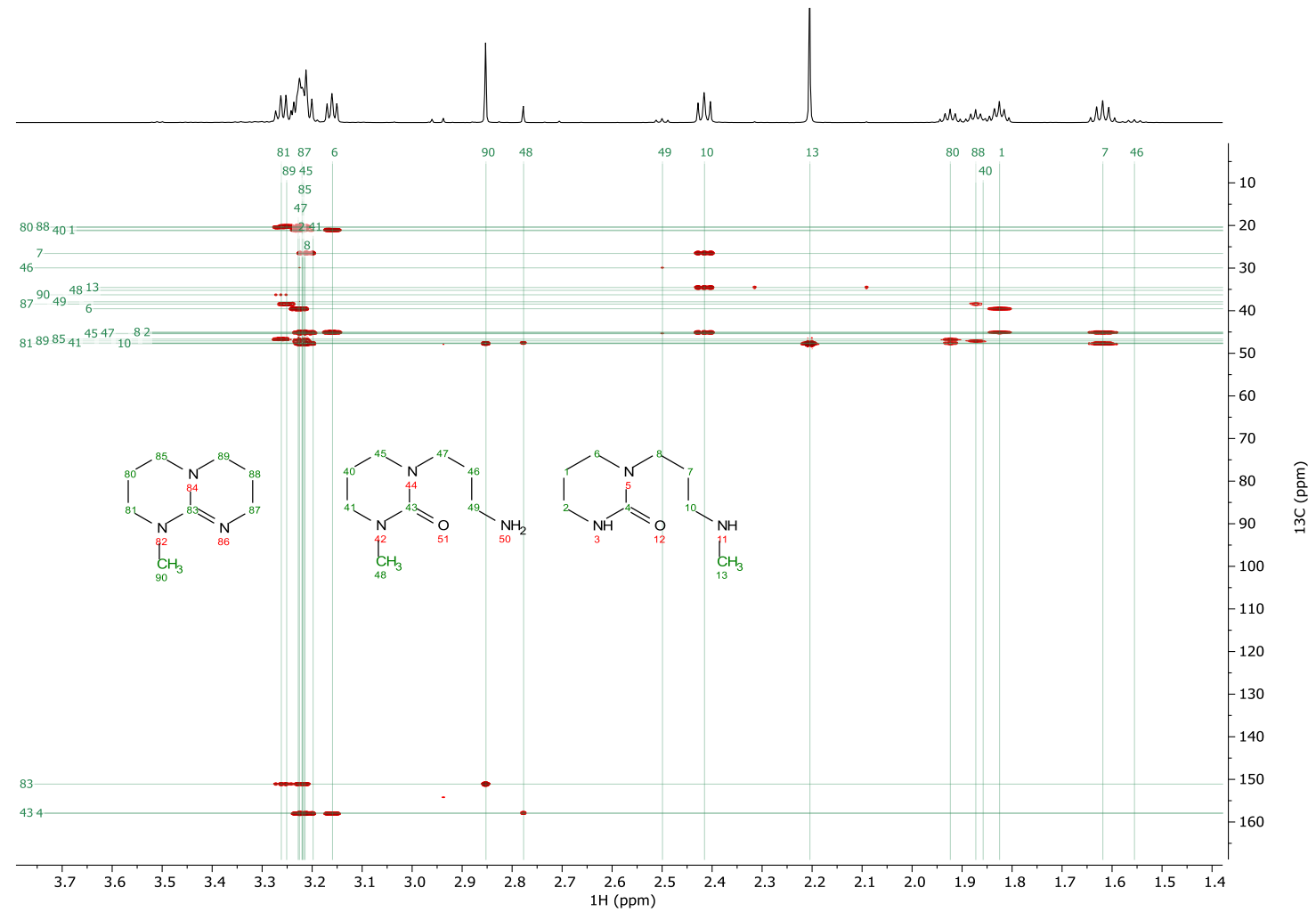


HSQC-TOCSY spectrum of partial hydrolysis mixture of MTBD $\left(D_{2} \mathrm{O}, 600 \mathrm{MHz}\right)$

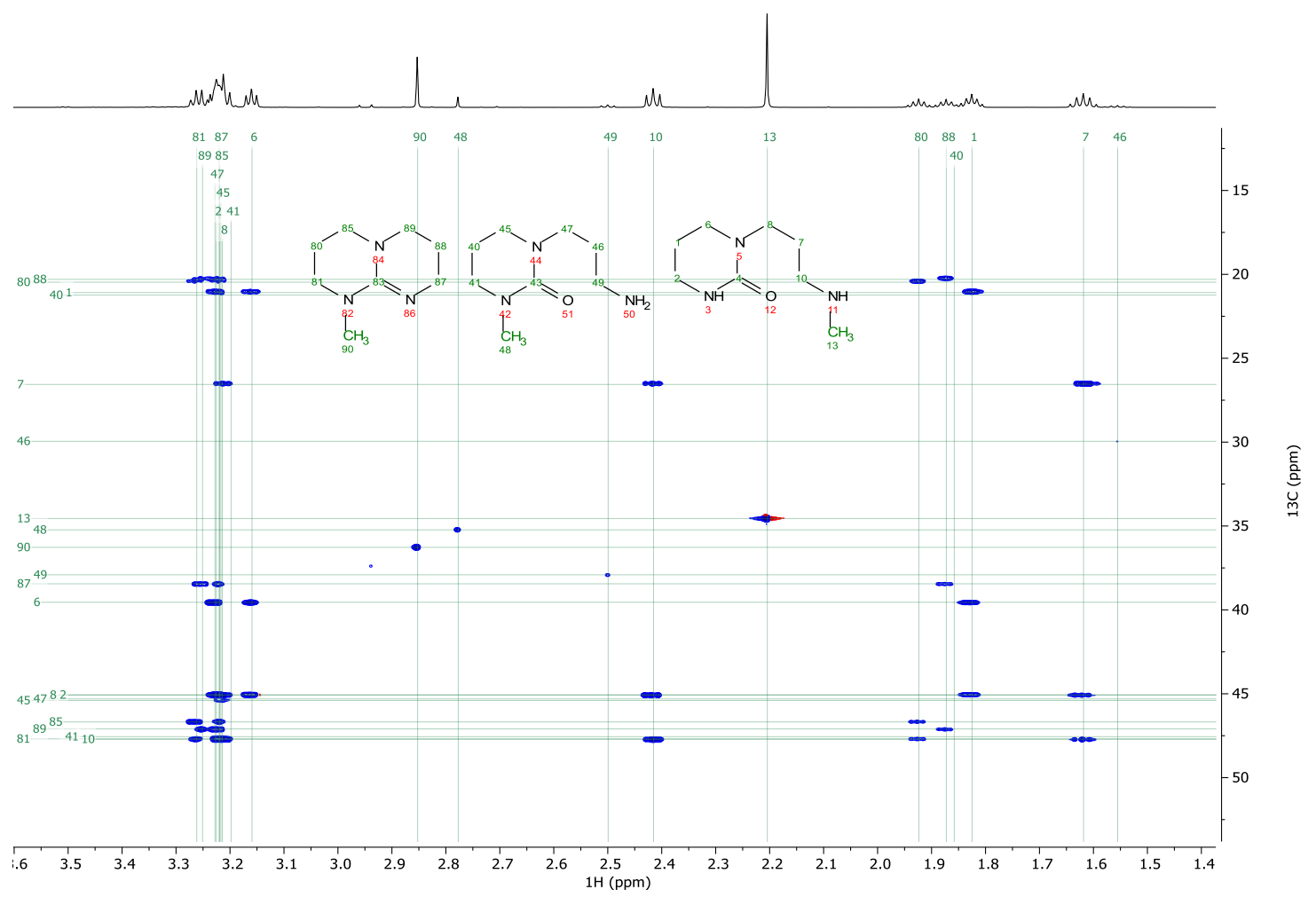

${ }^{1} \mathrm{H}$ spectrum of partial hydrolysis mixture of $\operatorname{TBD}\left(\mathrm{D}_{2} \mathrm{O}, 500 \mathrm{MHz}\right)$

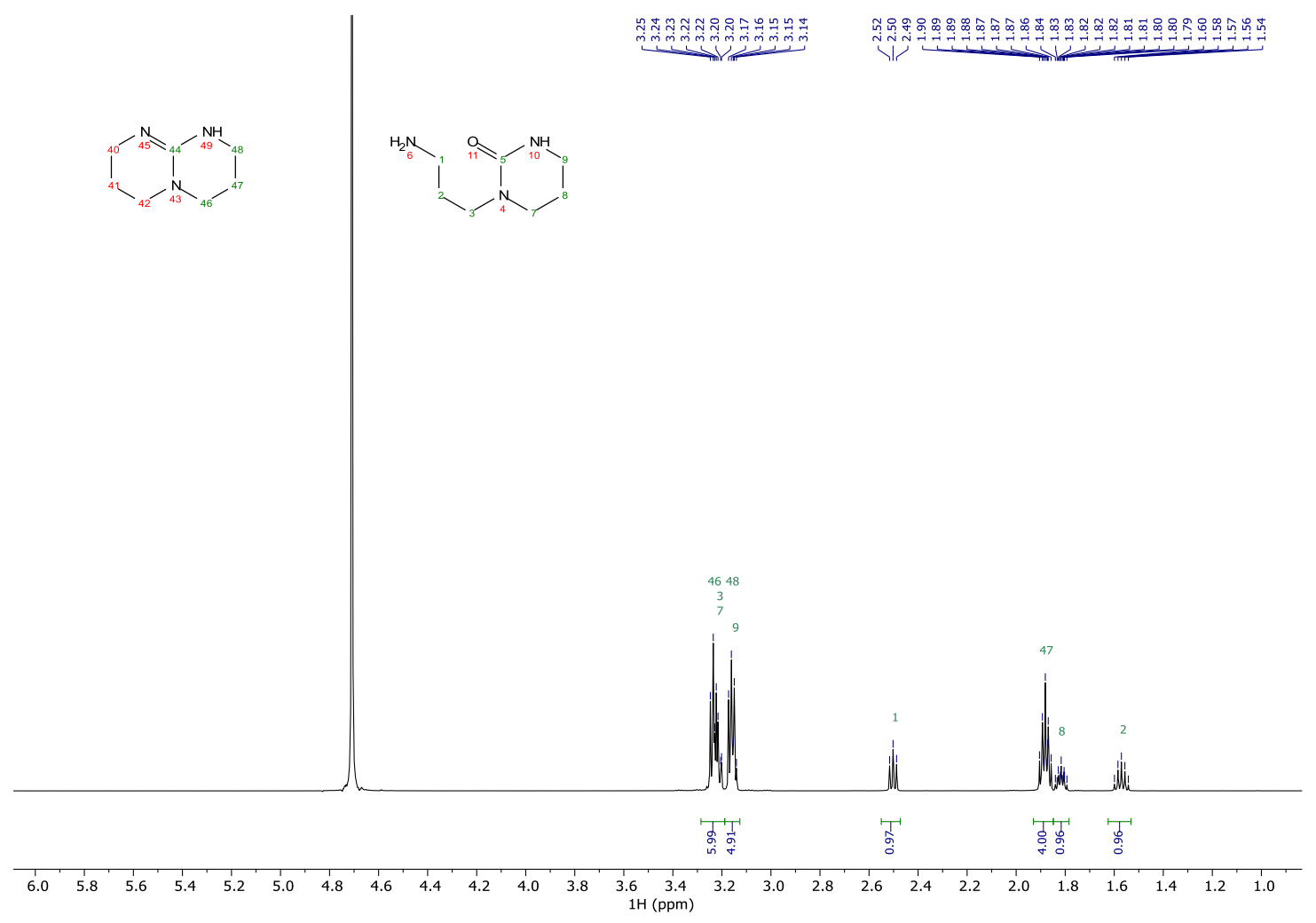


${ }^{13} \mathrm{C}\left\{{ }^{1} \mathrm{H}\right\}$ spectrum of partial hydrolysis mixture of $\operatorname{TBD}\left(\mathrm{D}_{2} \mathrm{O}, 126 \mathrm{MHz}\right)$

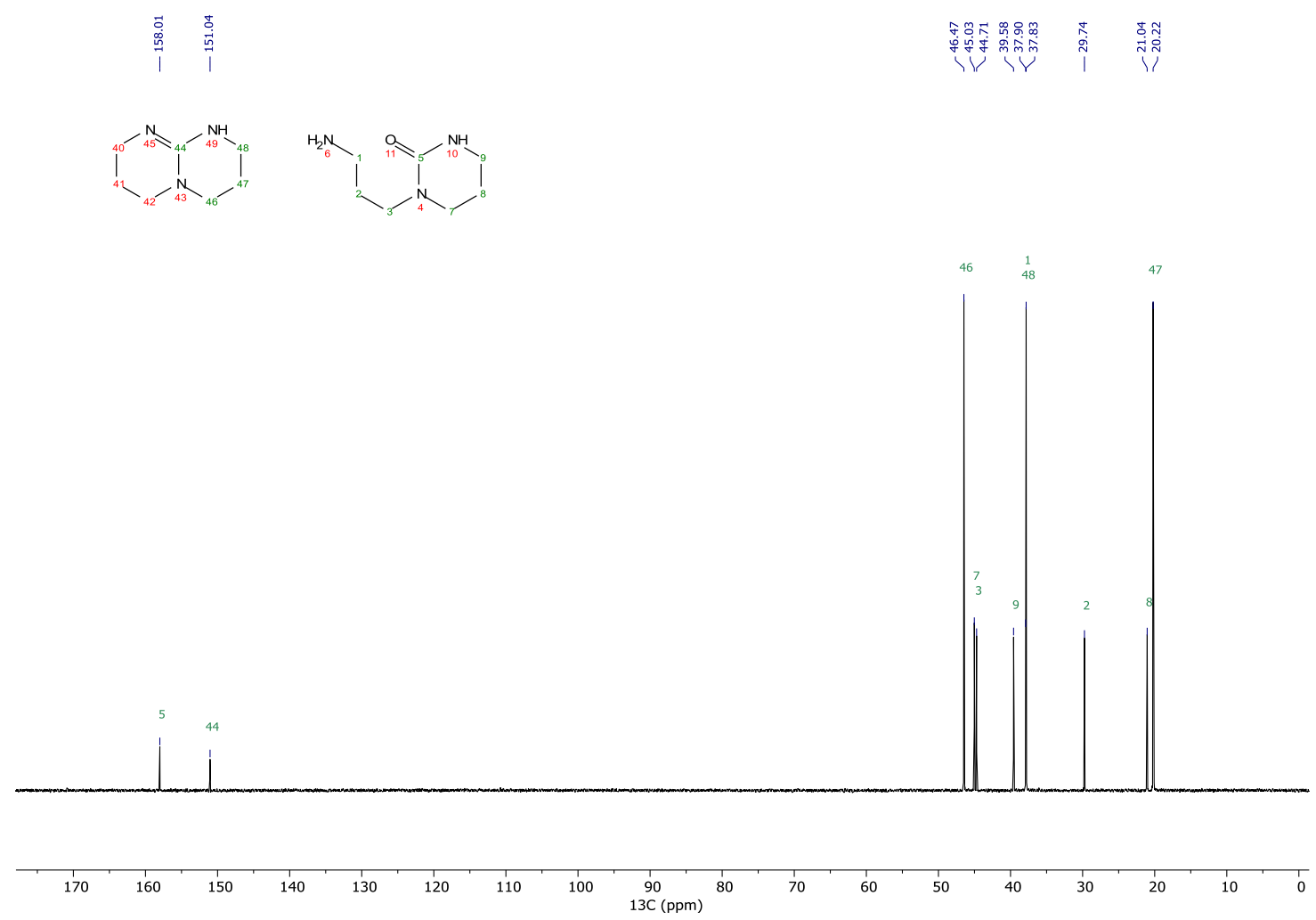

${ }^{1} \mathrm{H}-{ }^{1} \mathrm{H}$ COSY spectrum of partial hydrolysis mixture of $\operatorname{TBD}\left(\mathrm{D}_{2} \mathrm{O}, 500 \mathrm{MHz}\right)$

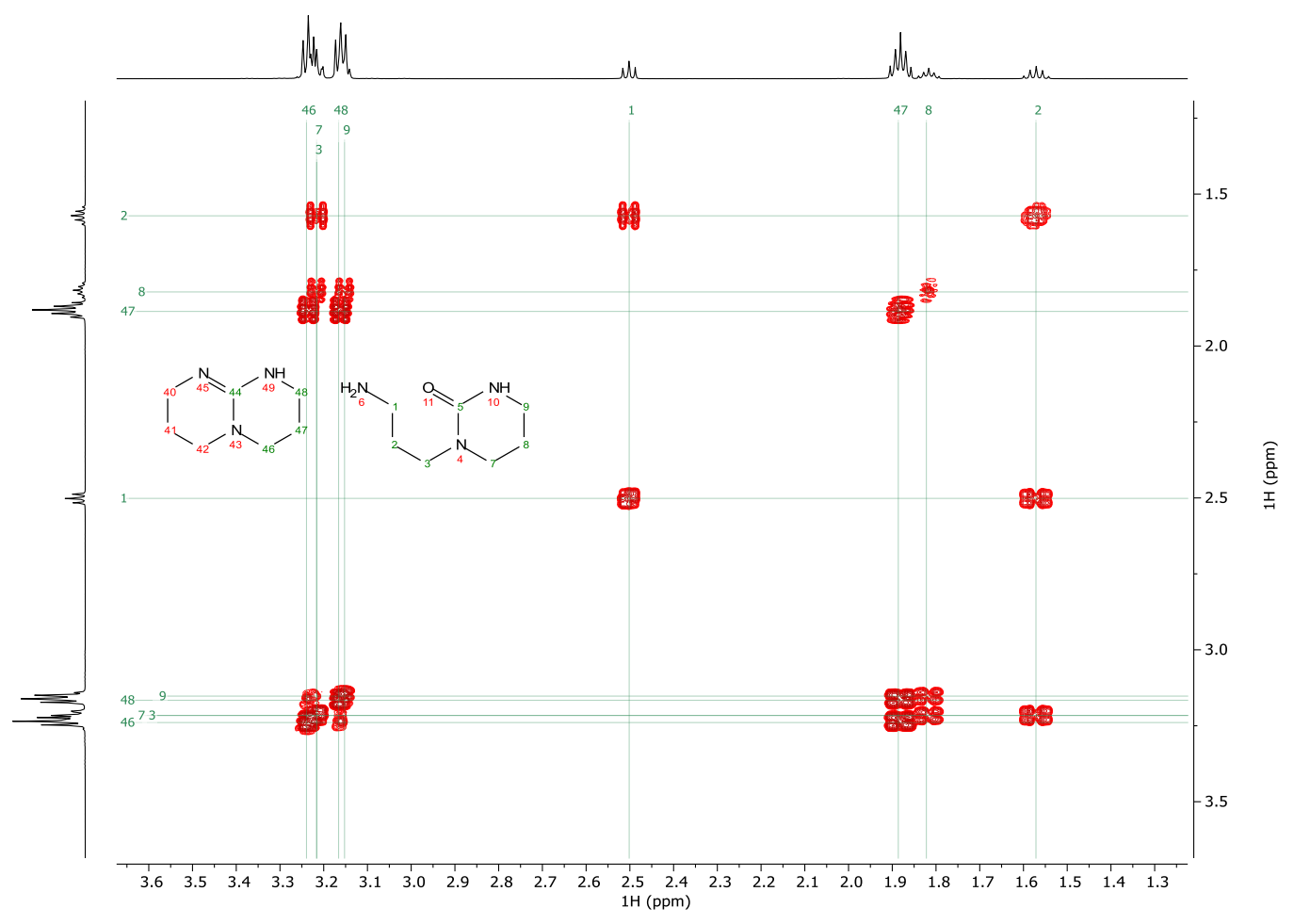


${ }^{1} \mathrm{H}-{ }^{13} \mathrm{C} \mathrm{HSQC}$ spectrum of partial hydrolysis mixture of $\operatorname{TBD}\left(\mathrm{D}_{2} \mathrm{O}, 500 \mathrm{MHz}\right)$

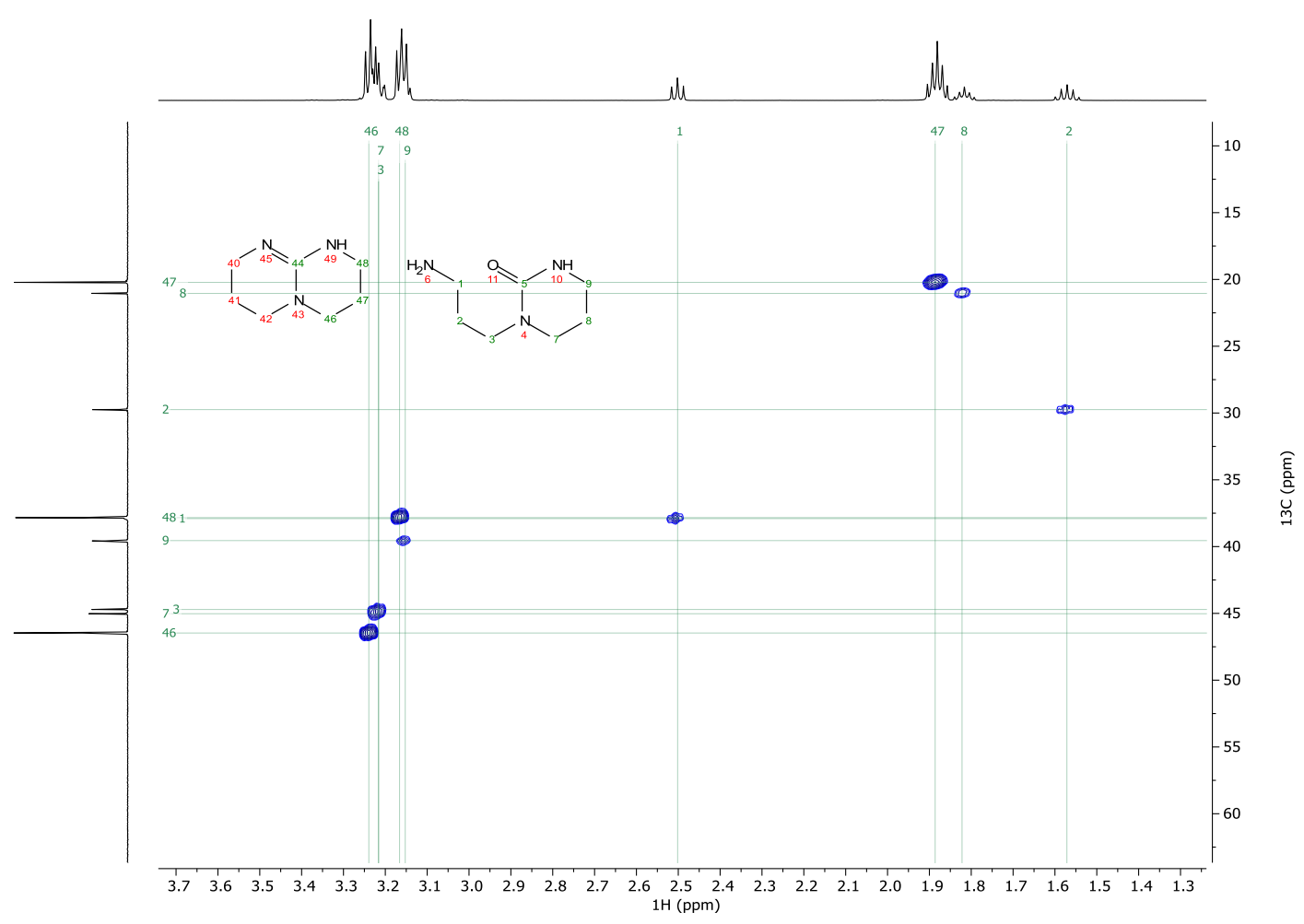

${ }^{1} \mathrm{H}-{ }^{13} \mathrm{C} \mathrm{HMBC}$ spectrum of partial hydrolysis mixture of $\operatorname{TBD}\left(\mathrm{D}_{2} \mathrm{O}, 500 \mathrm{MHz}\right)$

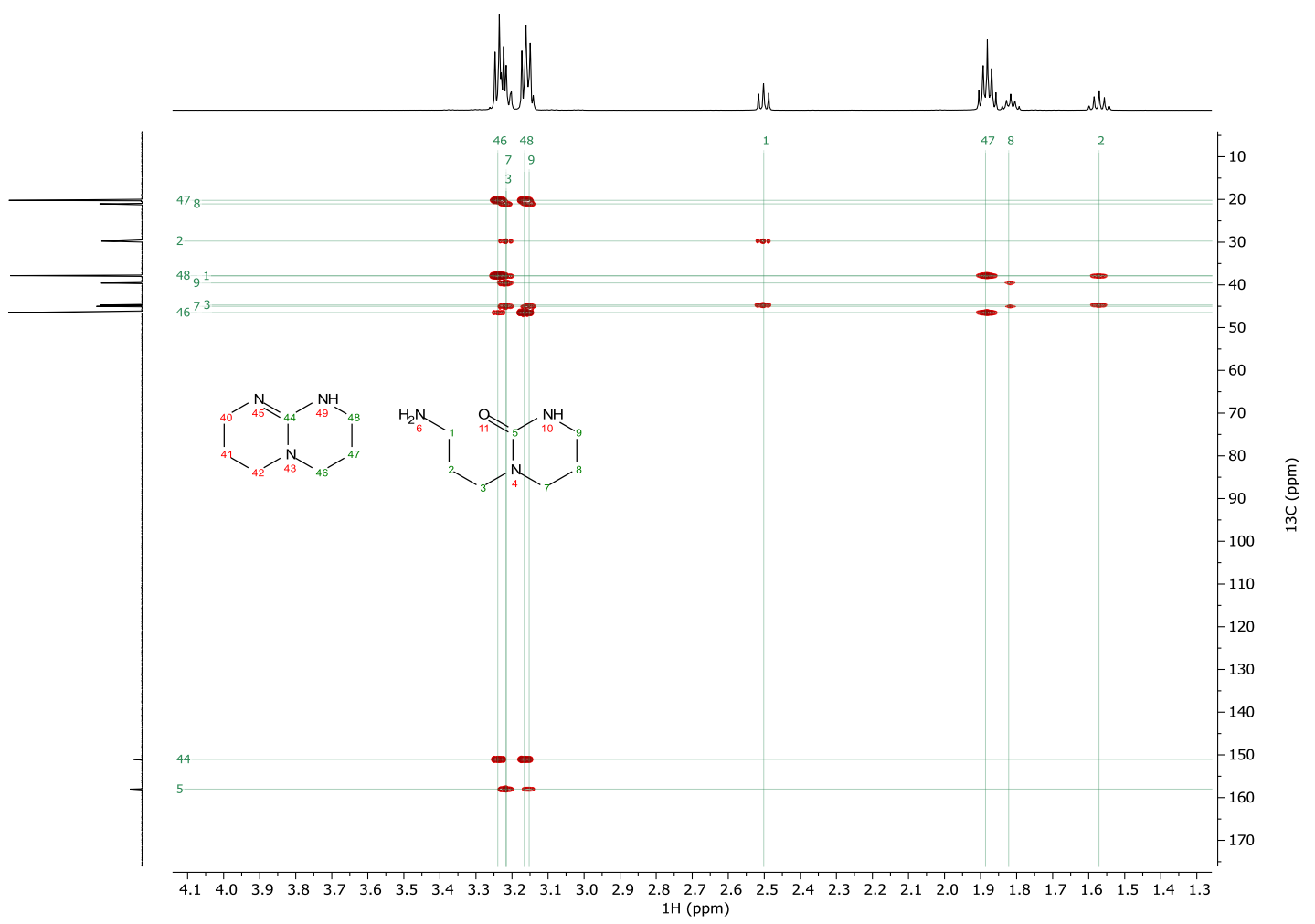


${ }^{1} \mathrm{H}$ spectrum of partial hydrolysis mixture of TMG $\left(\mathrm{D}_{2} \mathrm{O}, 500 \mathrm{MHz}\right)$

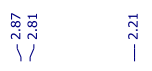<smiles>CN(C)C(=N)N(C)C</smiles><smiles>CN(C)C(N)=O</smiles>

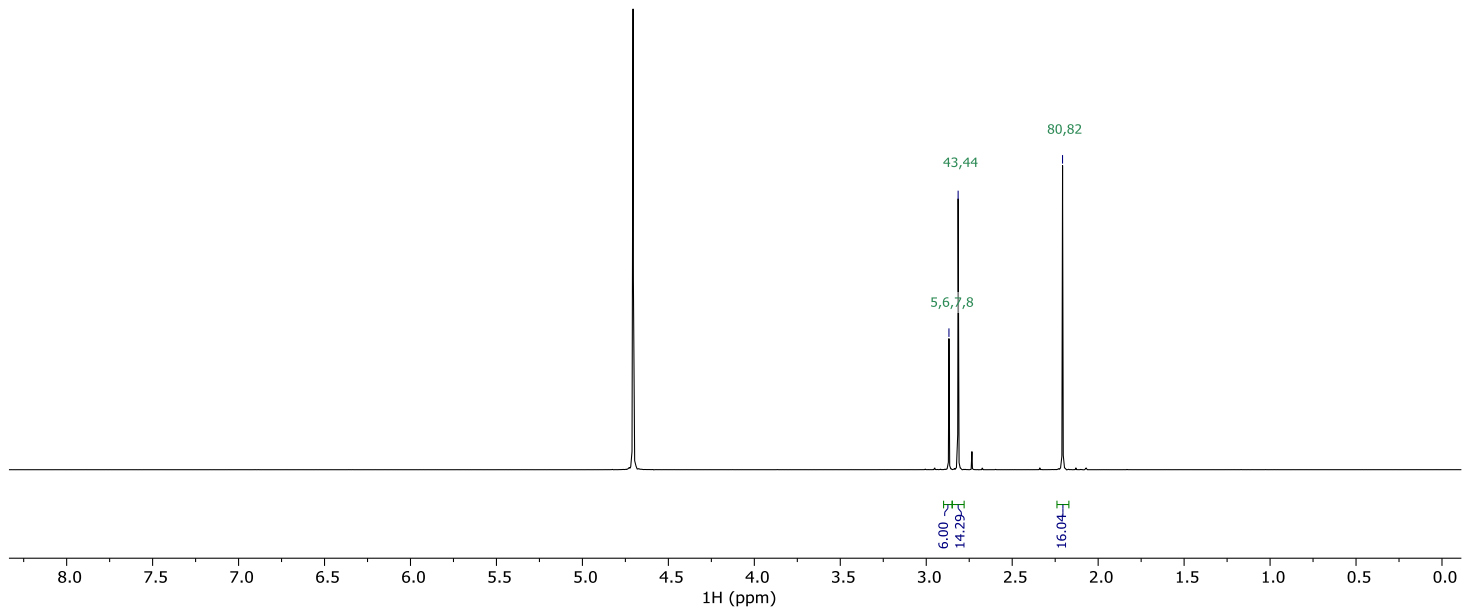

${ }^{13} \mathrm{C}$ spectrum of partial hydrolysis mixture of TMG $\left(\mathrm{D}_{2} \mathrm{O}, 126 \mathrm{MHz}\right)$

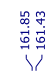<smiles>CN(C)C(=N)N(C)C</smiles><smiles>CN(C)C(N)=O</smiles><smiles>CN[13CH3]</smiles> 
${ }^{1} \mathrm{H}-{ }^{13} \mathrm{C} \mathrm{HSQC}$ spectrum of partial hydrolysis mixture of TMG $\left(\mathrm{D}_{2} \mathrm{O}, 500 \mathrm{MHz}\right)$

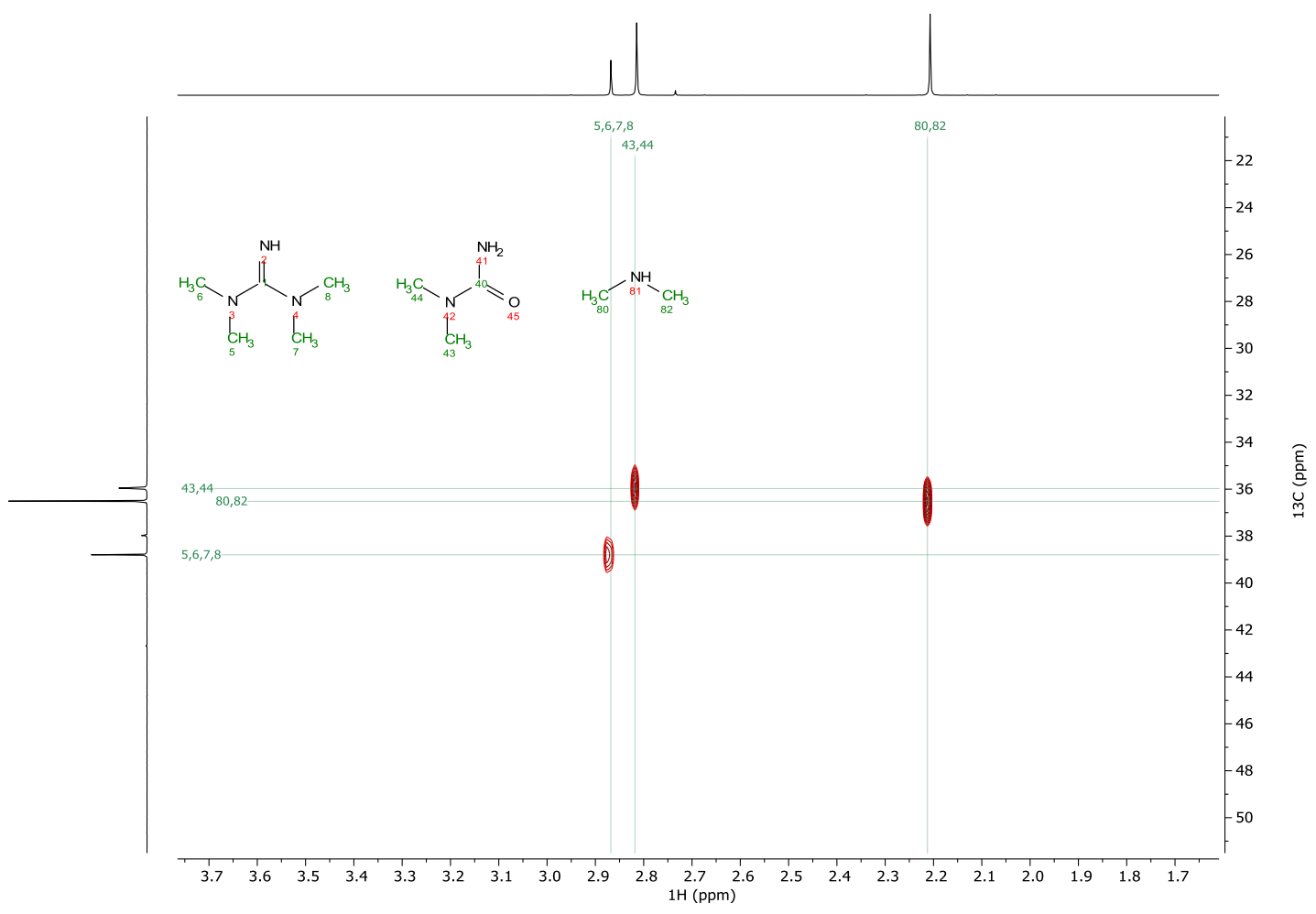

${ }^{1} \mathrm{H}-{ }^{13} \mathrm{C} \mathrm{HMBC}$ spectrum of partial hydrolysis mixture of TMG $\left(\mathrm{D}_{2} \mathrm{O}, 500 \mathrm{MHz}\right)$

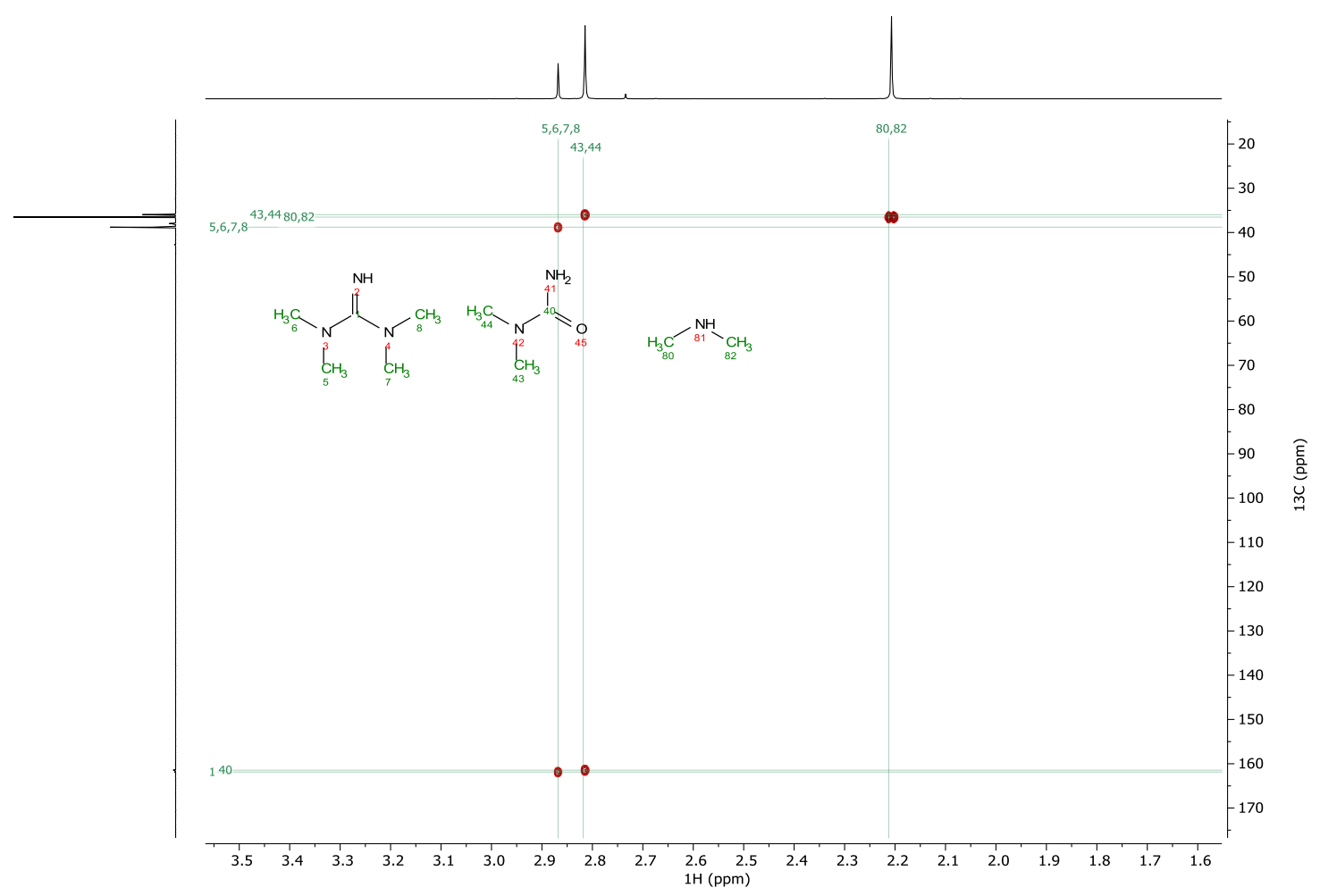

